\title{
Construction of Fused Oxabicyclic Scaffolds from Glycals and Styrenes via One-pot Domino Transformations
}

\author{
Monika Bhardwaj ${ }^{\dagger, \ddagger}$ Faheem Rasool, ${ }^{\dagger, \ddagger}$ Madhu Babu Tatina, ${ }^{\dagger}$ Debaraj Mukherjee ${ }^{\dagger,,^{*}}$ \\ $\dagger$ Natural Product Chemistry Division, Indian Institute of Integrative Medicine (IIIM), Jammu, India. \\ $\$$ Academy of Scientific and Innovative Research (AcSIR-IIIM), Jammu-180001, India.
}

\section{Supporting Information}

General Information: All reagents were purchased from commercial sources and used without treatment. Silica gel coated aluminum plates were used for TLC. The products were purified by column chromatography on silica gel (100-200 mesh) using hexane-ethyl acetate as the eluent. ${ }^{1} \mathrm{H}$ NMR (400 MHz) spectra were recorded on a Bruker Avance 400 spectrometer in $\mathrm{CDCl}_{3}$, using $\mathrm{CDCl}_{3}$ (for $1 \mathrm{H}, \delta=7.26$ ) as the internal standard. ${ }^{13} \mathrm{C}$ NMR $(101 \mathrm{MHz}$ ) spectra were recorded on a Bruker Avance 400 spectrometer in $\mathrm{CDCl}_{3}$ using $\mathrm{CDCl}_{3}$ (for ${ }^{13} \mathrm{C}, \delta=77.0$ ) as internal standard. Chemical shifts are expressed in parts per million $(\delta \mathrm{ppm})$. The following abbreviations were used to explain the multiplicities: $\mathrm{s}=$ singlet, $\mathrm{d}=$ doublet, $\mathrm{t}=$ triplet, $\mathrm{q}=$ quartet, $\mathrm{dd}=$ doublet of doublet, $\mathrm{ddd}=$ doublet of doublet of doublet, $\mathrm{dt}=$ doublet of triplet, $\mathrm{m}=$ multiplet, $\mathrm{s}$ br $=$ singlet broad. Exact mass of all products were analysed by using HRMS having QTOF analyser.

Table 1. Optimization of different solvent for the synthesis of cyclopenta[b]furan from glycals and styrenes. ${ }^{a}$

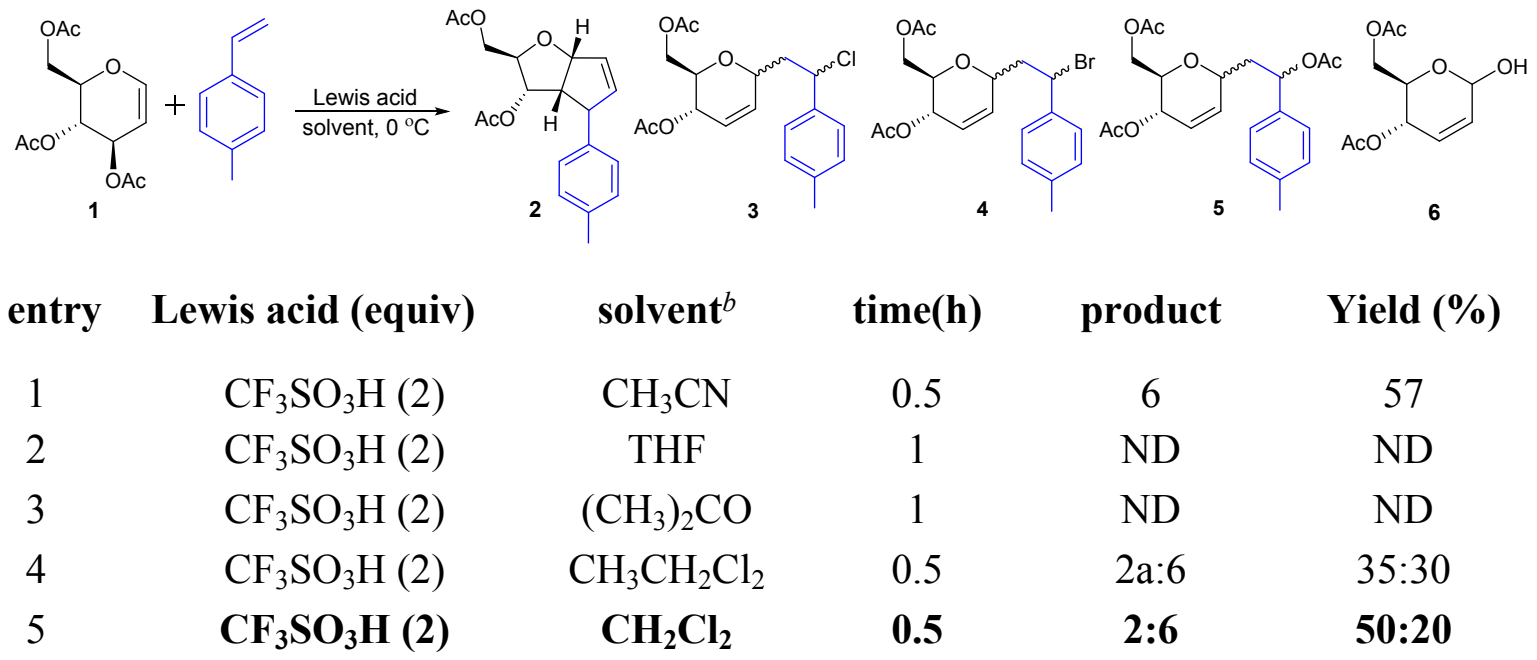


${ }^{a}$ all reaction are carried out by using tri- $O$-acetyl-D-glucal (1 equiv), 4-methylstyrene ( 1 equiv). ${ }^{b} 3$ $\mathrm{mL}$ solvent is used for $0.36 \mathrm{mmol}$ of tri- $O$-acetyl-D-glucal.

General experimental procedures for the synthesis of cyclopenta[b]furan (A).

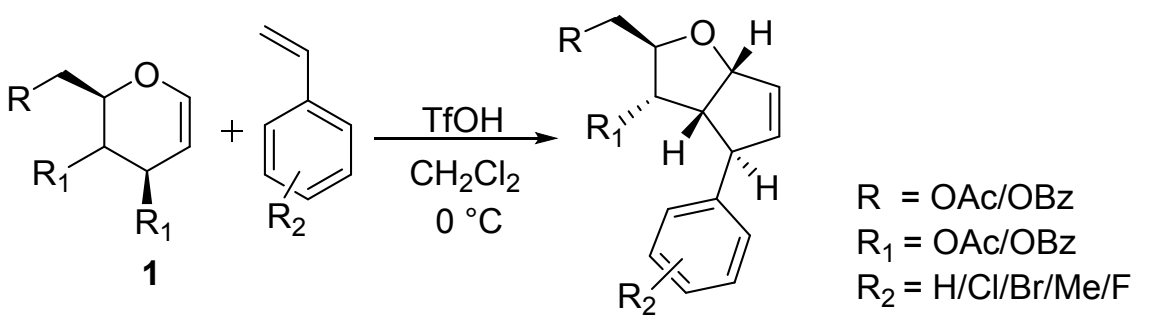

2-2k

To a solution of glycal (1 equiv) in $\mathrm{CH}_{2} \mathrm{Cl}_{2}(3 \mathrm{ml})$ taken in $25 \mathrm{~mL}$ oven dried single neck round bottom flask, Styrene ( 1 equiv) was added at $0{ }^{\circ} \mathrm{C}$, after 10 minutes of continuous stirring $\mathrm{CF}_{3} \mathrm{SO}_{3} \mathrm{H}$ (2 equiv) was added at the same temperature slowly. The mixture was stirred at $0{ }^{\circ} \mathrm{C}$ until TLC showed disappearance of glycal. The reaction was quenched with triethylamine ((5 equiv w.r.t $\mathrm{TfOH}$ ) and warmed to room temperature. The resulting mixture was extracted with $\mathrm{CH}_{2} \mathrm{Cl}_{2}$ and the combined organic extracts were washed with brine, dried over $\mathrm{Na}_{2} \mathrm{SO}_{4}$ and concentrated. The residue was purified by flash column chromatography (Hexane: EtOAc; 97:3) to afford the desired cyclopenta[ $[b]$ furan as oily product in moderate yields.

\section{Structure determination of compound 2:}

The structure of product 2 was confirmed by extensive NMR analysis. Presence of two new peaks at $\delta$ values $5.89 \mathrm{ppm}$ and $6.02 \mathrm{ppm}$ in $1 \mathrm{H}$ NMR suggested the presence of alkene proton. The coupling constant of $5.5 \mathrm{~Hz}$ was observed for an alkene proton which confirmed the presence of cyclopentene moiety. Two new peaks observed at $\delta$ values $51.35 \mathrm{ppm}$ and 50.34 ppm in the CMR corresponds to with $\mathrm{C} 2$ and C8 respectively. In NOESY experiment we further observed the correlation of $\mathrm{H} 8$ with $\mathrm{H} 11$ and $\mathrm{H} 13$ which was suggestive towards styrene attached to $\mathrm{C} 8$ carbon of cyclopentene ring. However, strong correlation between $\mathrm{H}_{1}-\mathrm{H}_{6}, \mathrm{H}_{2}-\mathrm{H}_{8}$ in the $1 \mathrm{H}-1 \mathrm{H}$ COSY confirmed their adjacent nature, showing the fusion of furan and cyclopentene ring. Strong correlation between $\mathrm{H} 6$ and $\mathrm{H} 8$ in the 1H-1H NOESY spectrum confirms their close proximity in space. Similarly strong correlation between $\mathrm{H} 2$ and H13 suggesting they are cis to each other, which is also supposed by the fact that most of the 5,5 fused rings have cis ring juncture. No correlation between $\mathrm{H} 1$ and H8 confirming they are on opposite side. Detailed 2D NMR analysis revealed final corrected structure of compound $\mathbf{2}$ with furano pentene core. The 
stereochemistry of compound $\mathbf{2}$ was determined by 2D-NOESY (Fig). Further, the structure of 2 was verified by stereospecific dihydroxylation of the furanopentene moiety by $\mathrm{OsO}_{4}-\mathrm{NMO}$.

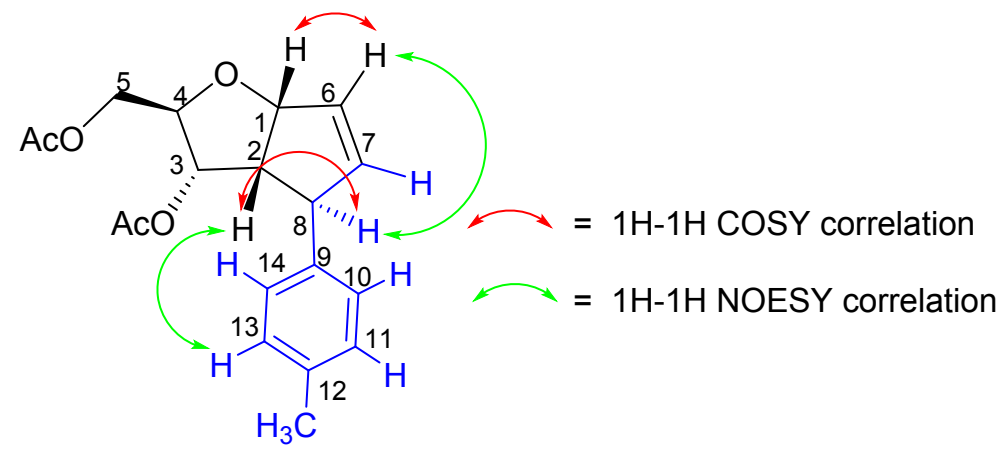

Figure 1. Various 1H-1H correlations in 2.

Peak assignments: ${ }^{1}$ H NMR $\delta 7.09(\mathrm{~d}, J=7.9 \mathrm{~Hz}, 2 \mathrm{H}, \mathrm{H}-10, \mathrm{H}-14), 6.93(\mathrm{~d}, J=8.0 \mathrm{~Hz}, 2 \mathrm{H}, \mathrm{H}-$ 11 and H-13), 6.03 (dd, $J=5.5,2.3 \mathrm{~Hz}, 1 \mathrm{H}, \mathrm{H}-7), 5.90$ (m, 1H, H-6), 5.44-5.35 (m, 1H, H-1), $5.01(\mathrm{t}, J=8.7 \mathrm{~Hz}, 1 \mathrm{H}, \mathrm{H}-3), 4.35$ (dd, $J=12.1,2.9 \mathrm{~Hz}, 1 \mathrm{H}, \mathrm{H}-5), 4.12(\mathrm{dd}, J=12.1,5.9 \mathrm{~Hz}, 1 \mathrm{H}$, H-5), 4.01-3.96 (m, 1H, H-8), 3.96-3.87 (m, 1H, H-4), 3.05-3.01 (m, 1H, H-2), 2.31 (s, 3H,), 2.16 (s, 3H- acetyl ), 2.10 (s, 3H- acetyl ). 13C NMR $\delta$ 170.8, 169.9, 140.6 (C-9), 139.6 (C-7), 136.3 (C-12), 130.1(C-6), 129.4 (C-10, C-14), 127.0 (C-11, C-13), 88.3 (C-1), 75.6 (C-4), 73.6 (C-3), 63.2 (C-5), 51.35 (C-2), 50.3 (C-8), 20.9, 20.8, 20.8.

\section{Structure determination of cis-oxadecalin 7:}

The formation of cyclised cis-oxadecalin product 7 was confirmed by extensive spectroscopic analysis and mass spectroscopy. The appearance of single acetyl peak peaks at $2.1(\mathrm{~s}, 3 \mathrm{H})$ and distinct difference of alkene protons i.e; H-4, 5.73 (d, J=10.3 Hz) and H-3, $\delta=6.3$ (bd) shows one of the proton (H-3) falls in aromatic anisotropy. Together with this, the downfield chemical shift of C-3 at $\delta=131 \mathrm{ppm}$ and appearance of one more aromatic quaternary carbon in the C-13 NMR shows the formation of benzanulated oxadecalin system. 2D spectra of 7 does not confirm anything as the important protons that is H-1, H-5 and H-6 absorb in the same region i.e; 4.41$4.34(\mathrm{~m}, 3 \mathrm{H})$, and no conclusion about the stereochemistry was drawn. Peak to peak assignment on the basis of $1 \mathrm{H}-1 \mathrm{H}$ cosy, HSQC is as; ${ }^{1} \mathbf{H}$ NMR (400 $\left.\mathbf{~ M H z}, \mathbf{C D C l}_{3}\right) \delta 7.37-7.32(\mathrm{~m}, 1 \mathrm{H})$, $7.30(\mathrm{~s}, 1 \mathrm{H}), 7.27(\mathrm{~s}, 1 \mathrm{H}), 7.21(\mathrm{~s}, 2 \mathrm{H}), 7.07(\mathrm{dd}, J=13.2,7.3 \mathrm{~Hz}, 3 \mathrm{H}), 6.85(\mathrm{~d}, J=7.7 \mathrm{~Hz}, 1 \mathrm{H})$, $6.30(\mathrm{dd}, J=10.3,3.0 \mathrm{~Hz}, 1 \mathrm{H} ; \mathrm{H}-3), 5.73$ (d, $J=10.3 \mathrm{~Hz}, 1 \mathrm{H} ; \mathrm{H}-4), 4.41-4.34$ (m, 3H; H1,8,6a), 4.30-4.25 (m, 1H; H-5), 4.11 (dd, $J=11.5,2.8$ Hz, 1H; H-6b), 3.59 (s, 1H; H-2), 2.452.39 (m, 1H; H-7a), 2.10 (s, 3H), 2.05 (bd, $J=10.6 \mathrm{~Hz}, 1 \mathrm{H} ; \mathrm{H}-7 \mathrm{~b}) .{ }^{13} \mathbf{C}$ NMR (101 MHz, $\left.\mathbf{C D C l}_{3}\right) \delta 171.0,146.1,138.3,137.8,131.1,129.8,128.8,128.3,128.3,126.6,126.2,126.1(\mathrm{C}-$ 3), 124.6 (C-4), 69.3 (C-1), 66.0 (C-5), 65.1 (C-6), 42.2 (C-8), 37.1 (C-2), 35.1 (C-7), 21.0. HRMS (ESI+): $\mathrm{m} / \mathrm{z}$ calcd. for $\mathrm{C}_{22} \mathrm{H}_{22} \mathrm{NaO}_{3}(\mathrm{M}+\mathrm{Na})^{+}: 357.1467$; found 357.1459 . 
The actual stereochemistry and regioselectivity of the cyclised cis-oxadecalin product was determined by 2D NMR of deacetylated product of $\mathbf{7 g}$ (below scheme), where all protons got resolved and absorb at different strengths.

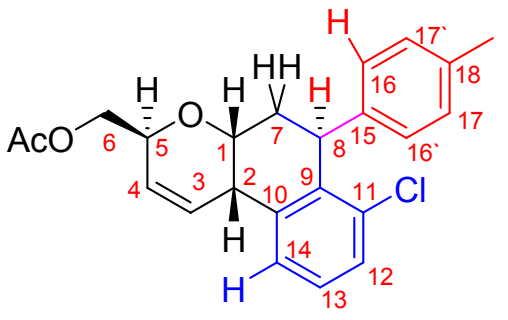

$7 g$

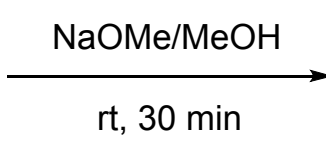

$\mathrm{rt}, 30 \mathrm{~min}$

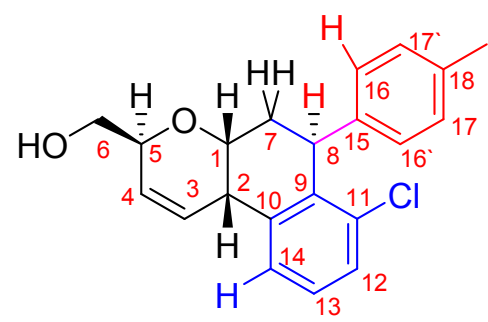

Scheme showing the deacetylation of $\mathbf{7 g}$.

Following assignments were given for different protons based on $1 \mathrm{H}$ NMR, 13C, $1 \mathrm{H}-1 \mathrm{H}$ cosy, HSQC etc

${ }^{1} \mathrm{H}$ NMR (400 MHz, $\mathrm{CDCl}_{3}$ ) $\delta 7.25$ (s, 3H; H-12,13,14), 7.04 (d, J=8.0 Hz, 2H; H-17a,b), 6.79 (d, $J=8.0 \mathrm{~Hz}, 2 \mathrm{H} ; \mathrm{H}-16 \mathrm{a}, \mathrm{b}), 5.88$ (dd, $J=10.3,0.9 \mathrm{~Hz}, 1 \mathrm{H} ; \mathrm{H}-3$ ), 5.64 (ddd, $J=10.4,2.6,1.6$ $\mathrm{Hz}, 1 \mathrm{H} ; \mathrm{H}-4), \underline{4.65-4.59}$ (dd, $J=5.3,1.8 \mathrm{~Hz}, 1 \mathrm{H} ; \mathrm{H}-8), \underline{4.34-4.19}(\mathrm{~m}, 1 \mathrm{H} ; \mathrm{H}-1), 4.19$ (dd, $J=3.6$, $1.8 \mathrm{~Hz}, 1 \mathrm{H} ; \mathrm{H}-5), 3.77$ (dd, $J=5.7,2.7 \mathrm{~Hz}, 1 \mathrm{H}), 3.70$ (dd, $J=11.3,3.1 \mathrm{~Hz}, 1 \mathrm{H} ; \mathrm{H}-6 \mathrm{a}), 3.57$ (dd, $J=11.3,6.1 \mathrm{~Hz}, 1 \mathrm{H} ; \mathrm{H}-6 \mathrm{~b}), 2.53-2.45$ (m, 1H; H-7a), 2.29 (s, 3H; Acetyl), 2.10 (s, 1H; H-7b).

The 1H-1H NOESY spectrum of the deacetylated compound of $7 \mathbf{g}$ (Fig. 2) shows a strong correlation between $\mathrm{H}-5$ and $\mathrm{H}-7 \mathrm{a}$, but not with $\mathrm{H}-1$ signifying that anomeric $\mathrm{H}$ is $\beta$. In the same manner correlation between $\mathrm{H}-1$ and $\mathrm{H}-16$ but not with $\mathrm{H}-8$ confirms $\mathrm{H}-8$ is $\beta$. No correlation between $\mathrm{H}-5$ and $\mathrm{H}-8$ with $\mathrm{H}-2$ points towards the $\alpha$-configuration of $\mathrm{H}-2$. The decalin system must be cis $(J=5.7 \mathrm{~Hz})$ as trans-fused ring will have both the ring juncture protons axially oriented showing higher coupling constant. The regioselectivity was determined from the HMBC where a strong correlation between $\mathrm{C}-2$ and $\mathrm{H}-12,13,15$ confirms that it is the 2-chlorophenyl ring and not 4-methylphenyl which is involved in the cyclization via second intramolecular Friedel-crafts reaction. 


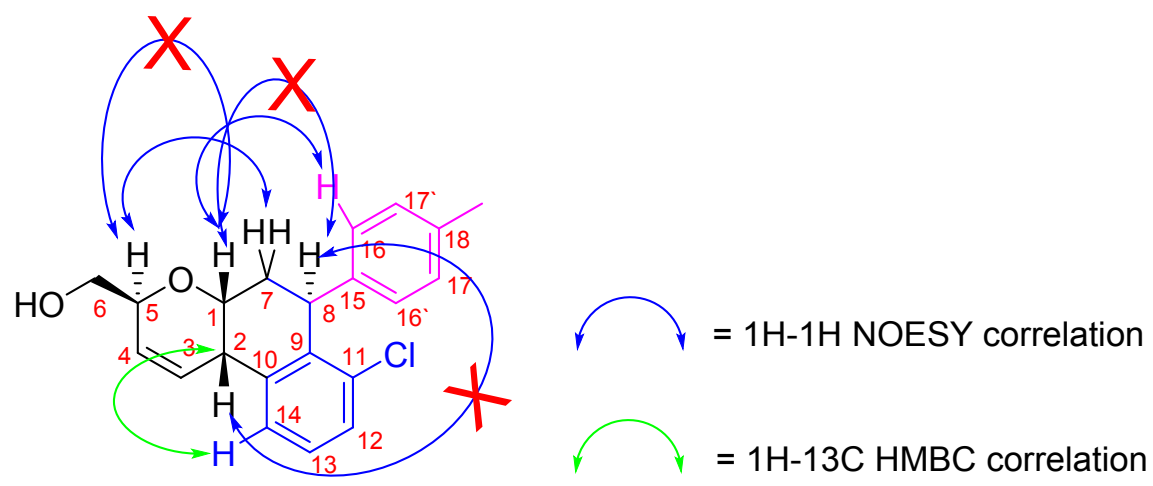

Figure 2: Various correlation in the deacetylated compound of $7 \mathrm{~g}$.

General experimental procedures for the synthesis of 1-oxadecalins from glycals and different styrenes (B).

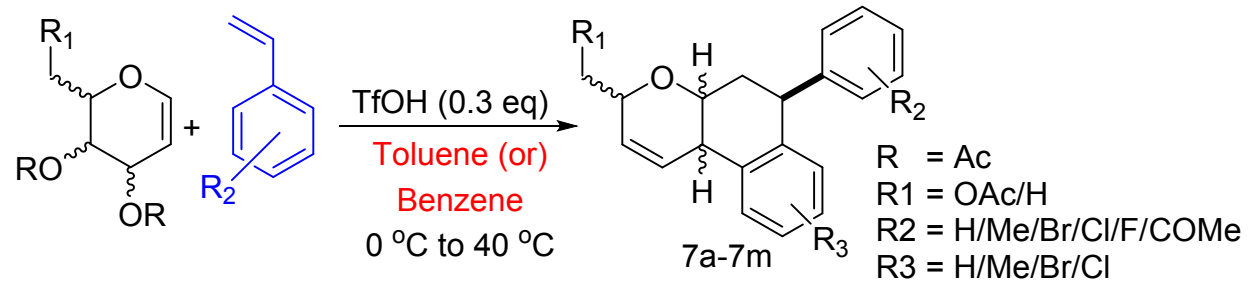

To a solution of glycal (1 equiv) in benzene $(3 \mathrm{~mL})$ taken in a $25 \mathrm{~mL}$ oven dried single neck round bottom flask, styrene ( 1 equiv) was added at $0{ }^{\circ} \mathrm{C}$ followed by slow addition of triflic acid ( 0.3 equiv) at the same temperature. After the addition is complete the reaction mixture was stirred at $40^{\circ} \mathrm{C}$ for $2 \mathrm{~h}$. After the completion of the reaction, then the reaction was quenched with triethylamine ( 5 equiv w.r.t $\mathrm{TfOH}$ ) at room temperature, thereafter the reaction mixture was concentrated. The resulting mixture was then extracted with $\mathrm{CH}_{2} \mathrm{Cl}_{2}$ and the combined organic extracts were washed with brine, dried over $\mathrm{Na}_{2} \mathrm{SO}_{4}$ and concentrated. The residue was purified 
by flash column chromatography (Hexane: EtOAc; 49:1) to afford the desired 1-oxadecalins as colourless oily compounds in good to excellent yields.

\section{Characterization of the products:}

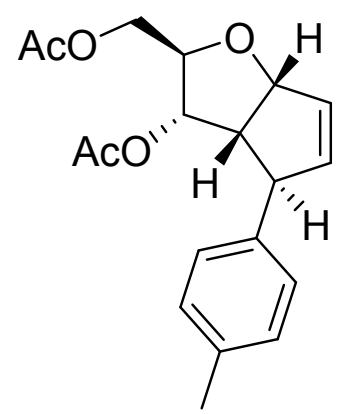

2

Prepared by general procedure A using tri- $O$-acetyl-D-glucal (272 mg, $1.0 \mathrm{mmol})$, 4-methyl styrene $(132.7 \mu \mathrm{L}, 1.0 \mathrm{mmol})$ and $\mathrm{CF}_{3} \mathrm{SO}_{3} \mathrm{H}(176.5 \mu \mathrm{L}, 2.0 \mathrm{mmol})$. The reaction mixture was stirred at $0{ }^{\circ} \mathrm{C}$ for 30 minutes, and then quenched with triethylamine. The resulting mixture was extracted with $\mathrm{CH}_{2} \mathrm{Cl}_{2}$ and the combined organic layer was washed with brine, dried over $\mathrm{MgSO}_{4}$ and concentrated. The residue was purified by flash column chromatography (Hexane: EtOAc; $97: 3)$ to afford 2 as yellow oil in 50\% yield, $165 \mathrm{mg}$.

${ }^{1}$ H NMR (400 MHz, $\left.\mathbf{C D C l}_{3}\right) \delta 7.09(\mathrm{~d}, J=7.9 \mathrm{~Hz}, 2 \mathrm{H}), 6.93(\mathrm{~d}, J=8.0 \mathrm{~Hz}, 2 \mathrm{H}), 6.03(\mathrm{dd}, J=$ 5.5, $2.3 \mathrm{~Hz}, 1 \mathrm{H}), 5.90(\mathrm{~m}, 1 \mathrm{H}), 5.44-5.35(\mathrm{~m}, 1 \mathrm{H}), 5.01(\mathrm{t}, J=8.7 \mathrm{~Hz}, 1 \mathrm{H}), 4.35(\mathrm{dd}, J=12.1$, $2.9 \mathrm{~Hz}, 1 \mathrm{H}), 4.12$ (dd, $J=12.1,5.9 \mathrm{~Hz}, 2 \mathrm{H}), 4.01-3.96(\mathrm{~m}, 1 \mathrm{H}), 3.96-3.87$ (m, 1H), 3.05-3.01 (m, 1H), 2.31 (s, 3H), 2.16 (s, 3H), 2.10 (s, 3H). ${ }^{13} \mathbf{C}$ NMR (101 MHz, CDCl $\left.\mathbf{3}\right) \delta 170.8,169.9$, 140.6, 139.6, 136.3, 130.1, 129.4, 127.0, 88.3, 75.6, 73.6, 63.2, 51.35, 50.3, 29.7, 20.9, 20.8, 20.8. HRMS (ESI+): m/z calcd. For $\mathrm{C}_{19} \mathrm{H}_{22} \mathrm{NaO}_{5}(\mathrm{M}+\mathrm{Na})^{+}: 353.1365$; found 353.1344.

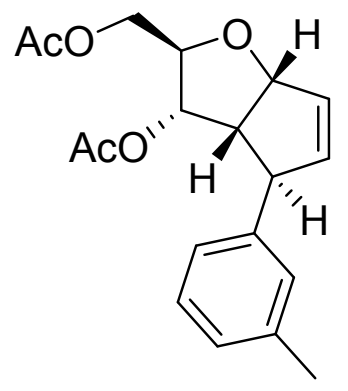


Prepared by general procedure A using tri- $O$-acetyl-D-glucal (100 mg, $0.36 \mathrm{mmol})$, 3-methyl styrene $(48.2 \mu \mathrm{L}, 0.36 \mathrm{mmol})$ and $\mathrm{CF}_{3} \mathrm{SO}_{3} \mathrm{H}(63.5 \mu \mathrm{L}, 0.72 \mathrm{mmol})$. The reaction mixture was stirred at $0{ }^{\circ} \mathrm{C}$ for 30 minutes, then quenched with triethylamine. The resulting mixture was extracted with $\mathrm{CH}_{2} \mathrm{Cl}_{2}$ and the combined organic layer was washed with brine, dried over $\mathrm{Na}_{2} \mathrm{SO}_{4}$ and concentrated. The residue was purified by flash column chromatography (Hexane: EtOAc; 97: 3) to afford 2a as yellow oil in 57\% yield, $68 \mathrm{mg}$.

${ }^{1}$ H NMR (400 MHz, CDCl $) \delta 7.20(\mathrm{t}, J=7.8 \mathrm{~Hz}, 1 \mathrm{H}), 7.05(\mathrm{~d}, J=7.5 \mathrm{~Hz}, 1 \mathrm{H}), 6.86(\mathrm{~d}, J=5.8$ $\mathrm{Hz}, 2 \mathrm{H}), 6.07(\mathrm{dd}, J=5.5,2.3 \mathrm{~Hz}, 1 \mathrm{H}), 5.97-5.91(\mathrm{~m}, 1 \mathrm{H}), 5.44(\mathrm{~d}, J=6.9 \mathrm{~Hz}, 1 \mathrm{H}), 5.06(\mathrm{t}, J=$ $8.6 \mathrm{~Hz}, 1 \mathrm{H}), 4.38$ (dd, $J=12.1,2.9 \mathrm{~Hz}, 1 \mathrm{H}), 4.15$ (dd, $J=12.1,5.9 \mathrm{~Hz}, 1 \mathrm{H}), 4.04-4.00$ (m, 1H), 3.97-3.93 (m, 1H), 3.09-3.04 (m, 1H), 2.34 (s, 3H), 2.19 (s, 3H), 2.12 (s, 3H). ${ }^{13}$ C NMR (101 MHz, $\left.\mathbf{C D C l}_{3}\right) \delta 170.8,169.9,143.6,139.5,138.3,130.2,128.6,127.8,127.4,124.2,88.3,75.7$, 73.5, 63.2, 51.2, 50.6, 29.7, 21.4, 20.8, 20.8. HRMS (ESI+): $\mathrm{m} / \mathrm{z}$ calcd. for $\mathrm{C}_{19} \mathrm{H}_{22} \mathrm{NaO}_{5}(\mathrm{M}+\mathrm{Na})^{+}$: 353.1365; found 353.1348.

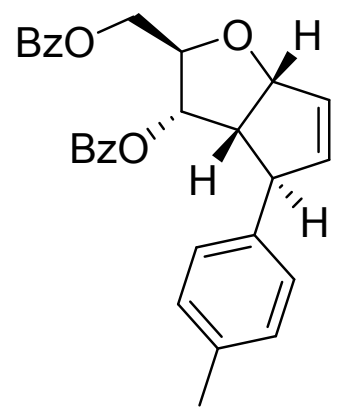

2b

Prepared by general procedure A using tri- $O$-benzoyl-D-glucal (100 mg, $0.22 \mathrm{mmol})$, 4methylstyrene $(32.8 \mu \mathrm{L}, 0.22 \mathrm{mmol})$ and $\mathrm{CF}_{3} \mathrm{SO}_{3} \mathrm{H}(38.8 \mu \mathrm{L}, 0.44 \mathrm{mmol})$. The reaction mixture was stirred at $0{ }^{\circ} \mathrm{C}$ for 30 minutes, and then quenched with triethylamine. The resulting mixture was extracted with $\mathrm{CH}_{2} \mathrm{Cl}_{2}$ and the combined organic layer was washed with brine, dried over $\mathrm{NaSO}_{4}$ and concentrated. The residue was purified by flash column chromatography (Hexane: EtOAc 97:3) to afford $\mathbf{2 b}$ as yellow oil in 56\% yield, $55 \mathrm{mg}$.

${ }^{1}$ H NMR (400 MHz, $\left.\mathbf{C D C l}_{3}\right) \delta 8.09(\mathrm{~d}, J=7.3 \mathrm{~Hz}, 2 \mathrm{H}), 8.03(\mathrm{~d}, J=7.3 \mathrm{~Hz}, 2 \mathrm{H}), 7.62(\mathrm{t}, J=7.4$ $\mathrm{Hz}, 1 \mathrm{H}), 7.54-7.45(\mathrm{~m}, 3 \mathrm{H}), 7.38(\mathrm{t}, J=7.7 \mathrm{~Hz}, 2 \mathrm{H}), 7.01(\mathrm{~d}, J=7.9 \mathrm{~Hz}, 2 \mathrm{H}), 6.86(\mathrm{~d}, J=7.9$ $\mathrm{Hz}, 2 \mathrm{H}), 6.07(\mathrm{dd}, J=5.4,2.2 \mathrm{~Hz}, 1 \mathrm{H}), 6.00-5.94(\mathrm{~m}, 1 \mathrm{H}), 5.54(\mathrm{~d}, J=6.9 \mathrm{~Hz}, 1 \mathrm{H}), 5.38(\mathrm{t}, J=$ $8.4 \mathrm{~Hz}, 1 \mathrm{H}), 4.72-4.63(\mathrm{~m}, 1 \mathrm{H}), 4.49(\mathrm{dd}, J=11.9,5.5 \mathrm{~Hz}, 1 \mathrm{H}), 4.29-4.22(\mathrm{~m}, 1 \mathrm{H}), 4.10$ (s, 1H), 3.24 - 3.18 (m, 1H), 2.28 (s, 3H). ${ }^{13} \mathbf{C}$ NMR (126 MHz, CDCl $\left.\mathbf{3}\right) \delta 166.38,165.68,140.59$, 139.47, 133.48, 133.08, 130.39, 129.77, 129.75, 129.32, 128.61, 128.33, 127.08, 88.76, 75.98, 74.80, 64.00, 51.74, 50.35, 20.99. HRMS (ESI+): $\mathrm{m} / \mathrm{z}$ calcd. for $\mathrm{C}_{29} \mathrm{H}_{27} \mathrm{O}_{5}(\mathrm{M}+\mathrm{H})^{+}:$455.1858; found 455.1855 


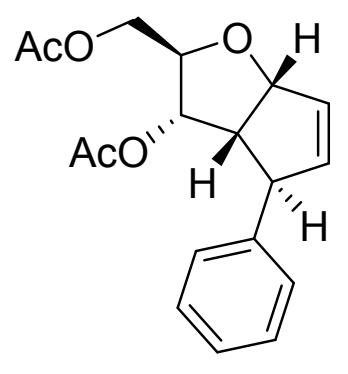

2c

Prepared by general procedure A using tri- $O$-acetyl-D-glucal (100 mg, $0.36 \mathrm{mmol})$, styrene (42.5 $\mu \mathrm{L}, 0.36 \mathrm{mmol})$ and $\mathrm{CF}_{3} \mathrm{SO}_{3} \mathrm{H}(63.51 \mu \mathrm{L}, 0.72 \mathrm{mmol})$. The reaction mixture was stirred at $0{ }^{\circ} \mathrm{C}$ for 30 minutes, and then quenched with triethylamine. The resulting mixture was extracted with $\mathrm{CH}_{2} \mathrm{Cl}_{2}$ and the combined organic layer was washed with brine, dried over $\mathrm{Na}_{2} \mathrm{SO}_{4}$ and concentrated. The residue was purified by flash column chromatography (Hexane: EtOAc 97:3) to afford $\mathbf{2 c}$ as yellow oil in $50 \%$ yield, $57 \mathrm{mg}$.

${ }^{1}$ H NMR (400 MHz, CDCl $) \delta$ 7.33-7.27 (m, 2H), 7.25-7.19 (m, 1H), 7.09-7.02 (m, 2H), 6.07 $(\mathrm{dd}, J=5.5,2.3 \mathrm{~Hz}, 1 \mathrm{H}), 5.94(\mathrm{dd}, J=3.4,2.2 \mathrm{~Hz}, 1 \mathrm{H}), 5.43(\mathrm{dd}, J=4.3,2.5 \mathrm{~Hz}, 1 \mathrm{H}), 5.03(\mathrm{t}, J$ $=8.7 \mathrm{~Hz}, 1 \mathrm{H}), 4.37(\mathrm{dd}, J=12.1,2.8 \mathrm{~Hz}, 1 \mathrm{H}), 4.14(\mathrm{dd}, J=12.1,5.9 \mathrm{~Hz}, 1 \mathrm{H}), 4.04(\mathrm{dd}, J=8.1$, $5.5 \mathrm{~Hz}, 1 \mathrm{H}), 3.98-3.92(\mathrm{~m}, 1 \mathrm{H}), 3.08-3.04(\mathrm{~m}, 1 \mathrm{H}), 2.18(\mathrm{~s}, 3 \mathrm{H}), 2.11(\mathrm{~s}, 3 \mathrm{H}) .{ }^{13} \mathrm{C}$ NMR (101 MHz, $\left.\mathbf{C D C l}_{3}\right) \delta 170.8,169.9,143.6,139.4,130.4,128.7,127.1,126.74,88.3,75.6,73.5,63.2$, 51.2, 50.7, 29.7, 20.9, 20.8. HRMS (ESI+): m/z calcd. for $\mathrm{C}_{18} \mathrm{H}_{20} \mathrm{NaO}_{5}(\mathrm{M}+\mathrm{Na})^{+}:$339.1208; found: 339.1210 .

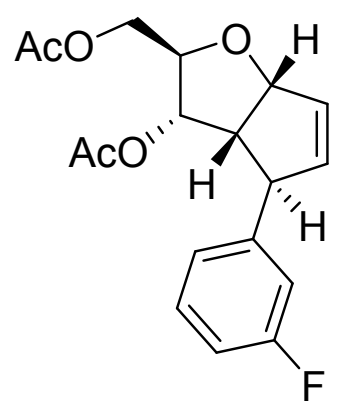

2d

Prepared by general procedure A using tri- $O$-acetyl-D-glucal (50 mg, $0.18 \mathrm{mmol}$ ), 3-fluoro styrene $(21.5 \mu \mathrm{L}, 0.18 \mathrm{mmol})$ and $\mathrm{CF}_{3} \mathrm{SO}_{3} \mathrm{H}(31.7 \mu \mathrm{L}, 0.36 \mathrm{mmol})$. The reaction mixture was stirred at $0^{\circ} \mathrm{C}$ for 30 minutes, and then quenched with triethylamine. The resulting mixture was extracted with $\mathrm{CH}_{2} \mathrm{Cl}_{2}$ and the combined organic layer was washed with brine, dried over 
$\mathrm{Na}_{2} \mathrm{SO}_{4}$ and concentrated. The residue was purified by flash column chromatography (Hexane: EtOAc; 97:3) to afford $2 \mathbf{d}$ as yellow oil in 36\% yield, $22 \mathrm{mg}$.

${ }^{1}$ H NMR (400 MHz, CDCl $) \delta 7.28-7.24(\mathrm{~m}, 1 \mathrm{H}), 6.94(\mathrm{dd}, J=8.4,2.0 \mathrm{~Hz}, 1 \mathrm{H}), 6.85(\mathrm{~d}, J=$ $7.7 \mathrm{~Hz}, 1 \mathrm{H}), 6.77$ (d, $J=9.9 \mathrm{~Hz}, 1 \mathrm{H}), 6.06$ (dd, $J=5.6,2.1 \mathrm{~Hz}, 1 \mathrm{H}), 6.01-5.93(\mathrm{~m}, 1 \mathrm{H}), 5.44$ (d, $J=6.9 \mathrm{~Hz}, 1 \mathrm{H}), 5.04(\mathrm{t}, J=8.7 \mathrm{~Hz}, 1 \mathrm{H}), 4.38(\mathrm{dd}, J=12.1,2.9 \mathrm{~Hz}, 1 \mathrm{H}), 4.16(\mathrm{dd}, J=12.1,5.8$ Hz, 1H), 4.04 (s, 1H), 3.94 (ddd, $J=8.6,5.8,2.9 \mathrm{~Hz}, 1 \mathrm{H}), 3.11-3.01(\mathrm{~m}, 1 \mathrm{H}), 2.20(\mathrm{~s}, 3 \mathrm{H}), 2.13$ (s, 3H). ${ }^{13} \mathbf{C}$ NMR (101 MHz, $\left.\mathbf{C D C l}_{3}\right) \quad \delta$ 170.80, 169.91, 138.69, 131.07, 130.24, 130.16, $122.84,122.81,114.18,113.96,113.77,113.56,88.18,75.67,73.53,63.17,51.20,50.45,22.69$, 20.83, 20.79. HRMS (ESI+): $\mathrm{m} / \mathrm{z}$ calcd. For $\mathrm{C}_{18} \mathrm{H}_{19} \mathrm{FNaO}_{5}(\mathrm{M}+\mathrm{Na})^{+}: 357.1114$; found 357.1093.

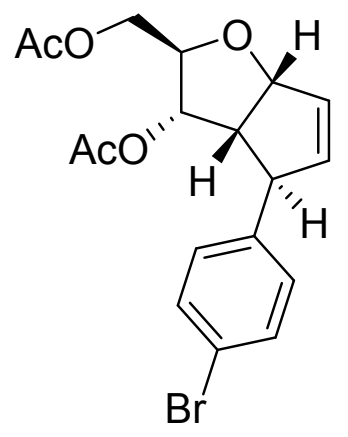

$2 e$

Prepared by general procedure A using tri- $O$-acetyl-D-glucal (100 mg, $0.36 \mathrm{mmol})$, 4-bromo styrene $(94.1 \mu \mathrm{L}, 0.36 \mathrm{mmol})$ and $\mathrm{CF}_{3} \mathrm{SO}_{3} \mathrm{H}(63.5 \mu \mathrm{L}, 0.72 \mathrm{mmol})$. The reaction mixture was stirred at $0{ }^{\circ} \mathrm{C}$ for 30 minutes, and then quenched with triethylamine. The resulting mixture was extracted with $\mathrm{CH}_{2} \mathrm{Cl}_{2}$ and the combined organic layer was washed with brine, dried over $\mathrm{Na}_{2} \mathrm{SO}_{4}$ and concentrated. The residue was purified by flash column chromatography (Hexane: EtOAc; $97: 3$ ) to afford $2 \mathrm{e}$ as yellow oil in $48 \%$ yield, $68 \mathrm{mg}$.

${ }^{1}$ H NMR (400 MHz, $\left.\mathbf{C D C l}_{3}\right) \delta 7.40(\mathrm{~d}, J=8.3 \mathrm{~Hz}, 2 \mathrm{H}), 6.91(\mathrm{~d}, J=8.3 \mathrm{~Hz}, 2 \mathrm{H}), 6.04-5.98$ $(\mathrm{m}, 1 \mathrm{H}), 5.96-5.91(\mathrm{~m}, 1 \mathrm{H}), 5.40(\mathrm{~d}, J=7.0 \mathrm{~Hz}, 1 \mathrm{H}), 4.98(\mathrm{t}, J=8.7 \mathrm{~Hz}, 1 \mathrm{H}), 4.36(\mathrm{dd}, J=$ $12.1,2.8 \mathrm{~Hz}, 1 \mathrm{H}), 4.12(\mathrm{dd}, J=12.1,5.8 \mathrm{~Hz}, 1 \mathrm{H}), 3.96(\mathrm{~s}, 1 \mathrm{H}), 3.93-3.88(\mathrm{~m}, 1 \mathrm{H}), 3.06-2.96$ (m, 1H), 2.17 (s, 3H), 2.10 (s, 3H). ${ }^{13} \mathbf{C}$ NMR (101 MHz, CDCl $\mathbf{~}_{3} \quad \delta$ 170.85, 169.95, 142.71, 138.83, 131.86, 131.80, 131.00, 128.88, 120.55, 88.26, 75.67, 75.58, 73.60, 73.56, 63.15, 51.20, 50.22, 22.70, 20.79. HRMS (ESI+): m/z calcd. For $\mathrm{C}_{18} \mathrm{H}_{19} \mathrm{BrNaO}_{5}(\mathrm{M}+\mathrm{Na})^{+}:$417.0314; found 417.0286 . 


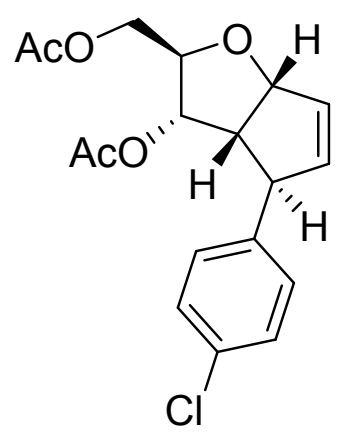

$2 f$

Prepared by general procedure A using tri- $O$-acetyl-D-glucal (100 mg, $0.36 \mathrm{mmol})$, 4-chloro styrene $(86.7 \mu \mathrm{L}, 0.36 \mathrm{mmol})$ and $\mathrm{CF}_{3} \mathrm{SO}_{3} \mathrm{H}(63.5 \mu \mathrm{L}, 0.72 \mathrm{mmol})$. The reaction mixture was stirred at $0{ }^{\circ} \mathrm{C}$ for 30 minutes, and then quenched with triethylamine. The resulting mixture was extracted with $\mathrm{CH}_{2} \mathrm{Cl}_{2}$ and the combined organic layer was washed with brine, dried over $\mathrm{Na}_{2} \mathrm{SO}_{4}$ and concentrated. The residue was purified by flash column chromatography (Hexane: EtOAc; 97:3) to afford $\mathbf{2 f}$ as yellow oil in 53\% yield, $66 \mathrm{mg}$.

${ }^{1}$ H NMR (400 MHz, CDCl $\left.{ }_{3}\right) \delta 7.35-7.28(\mathrm{~m}, 1 \mathrm{H}), 7.24(\mathrm{~d}, J=1.9 \mathrm{~Hz}, 1 \mathrm{H}), 6.99-6.93(\mathrm{~m}$, 2H), $6.01(\mathrm{dd}, J=5.5,2.2 \mathrm{~Hz}, 1 \mathrm{H}), 5.95-5.92(\mathrm{~m}, 1 \mathrm{H}), 5.40(\mathrm{~d}, J=6.8 \mathrm{~Hz}, 1 \mathrm{H}), 4.98(\mathrm{t}, J=8.7$ $\mathrm{Hz}, 1 \mathrm{H}), 4.35(\mathrm{dd}, J=12.1,2.7 \mathrm{~Hz}, 1 \mathrm{H}), 4.12(\mathrm{dd}, J=12.1,5.8 \mathrm{~Hz}, 1 \mathrm{H}), 3.99-3.95(\mathrm{~m}, 1 \mathrm{H})$, 3.91 (ddd, $J=8.6,5.8,2.8 \mathrm{~Hz}, 1 \mathrm{H}), 3.05-2.98(\mathrm{~m}, 1 \mathrm{H}), 2.16(\mathrm{~s}, 3 \mathrm{H}), 2.10(\mathrm{~s}, 3 \mathrm{H}) .{ }^{13} \mathbf{C} \mathbf{~ N M R}$ $\left(\mathbf{1 0 1} \mathbf{~ M H z}, \mathbf{C D C l}_{3}\right) \delta 170.8,169.9,142.1,138.8,132.5,130.9,128.8,128.5,88.2,75.6,73.6$, 63.1, 51.2, 50.1, 29.6, 20.8, 20.8. HRMS (ESI+): m/z calcd. For $\mathrm{C}_{18} \mathrm{H}_{19} \mathrm{ClNaO}_{5}(\mathrm{M}+\mathrm{Na})^{+}$: 373.0819; found 373.0794 .

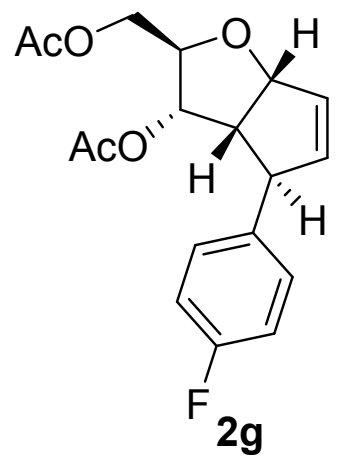

Prepared by general procedure A using tri- $O$-acetyl-D-glucal (100 mg, $0.36 \mathrm{mmol}$ ), 4-fluoro styrene $(43.5 \mu \mathrm{L}, 0.36 \mathrm{mmol})$ and $\mathrm{CF}_{3} \mathrm{SO}_{3} \mathrm{H}(63.5 \mu \mathrm{L}, 0.72 \mathrm{mmol})$. The reaction mixture was stirred at $0{ }^{\circ} \mathrm{C}$ for 30 minutes, and then quenched with triethylamine. The resulting mixture was extracted with $\mathrm{CH}_{2} \mathrm{Cl}_{2}$ and the combined organic layer was washed with brine, dried over $\mathrm{Na}_{2} \mathrm{SO}_{4}$ and concentrated. The residue was purified by flash column chromatography (Hexane: EtOAc; $97: 3$ ) to afford $2 \mathrm{~g}$ as yellow oil in $41 \%$ yield, $49 \mathrm{mg}$. 
${ }^{1}$ H NMR (400 MHz, $\left.\mathbf{C D C l}_{3}\right) \delta$ 7.04-6.93 (m, 4H), $6.02(\mathrm{dd}, J=5.5,2.3 \mathrm{~Hz}, 1 \mathrm{H}), 5.93-5.91$ (m, $1 \mathrm{H}), 5.40(\mathrm{~d}, J=6.9 \mathrm{~Hz}, 1 \mathrm{H}), 4.99(\mathrm{t}, J=8.7 \mathrm{~Hz}, 1 \mathrm{H}), 4.35(\mathrm{dd}, J=12.1,2.8 \mathrm{~Hz}, 1 \mathrm{H}), 4.12(\mathrm{dd}$, $J=12.1,5.8 \mathrm{~Hz}, 1 \mathrm{H}), 4.01-3.97$ (m, 1H), 3.94-3.89 (m, 1H), 3.03-2.98 (m, 1H), 2.17 (s, 3H), 2.09 (s, 3H). ${ }^{13} \mathbf{C}$ NMR (101 MHz, $\left.\mathbf{C D C l}_{3}\right) \delta 170.8,169.9,139.1,130.6,128.6,128.5,115.6$, 115.4, 88.2, 75.6, 73.6, 63.1, 51.3, 50.0 29.7, 20.8, 20.8. HRMS (ESI+): m/z calcd. For $\mathrm{C}_{18} \mathrm{H}_{19} \mathrm{FNaO}_{5}(\mathrm{M}+\mathrm{Na})^{+}:$357.1114; found 357.1123.

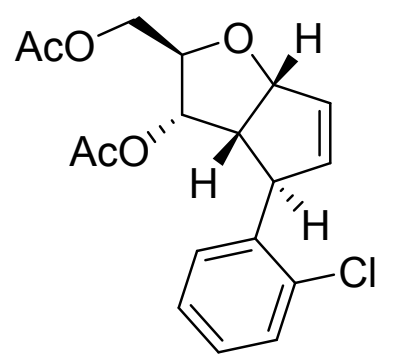

$2 \mathrm{~h}$

Prepared by general procedure A using tri- $O$-acetyl-D-glucal $(50 \mathrm{mg}, 0.18 \mathrm{mmol})$, 2-chloro styrene $(22.7 \mu \mathrm{L}, 0.18 \mathrm{mmol})$ and $\mathrm{CF}_{3} \mathrm{SO}_{3} \mathrm{H}(31.7 \mu \mathrm{L}, 0.36 \mathrm{mmol})$. The reaction mixture was stirred at $0{ }^{\circ} \mathrm{C}$ for 30 minutes, and then quenched with triethylamine. The resulting mixture was extracted with $\mathrm{CH}_{2} \mathrm{Cl}_{2}$ and the combined organic layer was washed with brine, dried over $\mathrm{Na}_{2} \mathrm{SO}_{4}$ and concentrated. The residue was purified by flash column chromatography (Hexane: EtOAc; $97: 3$ ) to afford $\mathbf{2 h}$ as yellow oil in $41 \%$ yield, $26 \mathrm{mg}$.

${ }^{1}$ H NMR (400 MHz, $\left.\mathbf{C D C l}_{3}\right) \delta 7.36(\mathrm{dd}, J=7.8,1.3 \mathrm{~Hz}, 1 \mathrm{H}), 7.21(\mathrm{dd}, J=7.4,1.4 \mathrm{~Hz}, 1 \mathrm{H})$, $7.16(\mathrm{dd}, J=7.5,1.8 \mathrm{~Hz}, 1 \mathrm{H}), 7.04(\mathrm{dd}, J=7.5,1.7 \mathrm{~Hz}, 1 \mathrm{H}), 6.01$ (dd, $J=5.5,2.2 \mathrm{~Hz}, 1 \mathrm{H}), 5.98$ $(\mathrm{m}, 1 \mathrm{H}), 5.50(\mathrm{~d}, J=7.0 \mathrm{~Hz}, 1 \mathrm{H}), 5.10(\mathrm{t}, J=8.5 \mathrm{~Hz}, 1 \mathrm{H}), 4.67-4.60(\mathrm{~m}, 1 \mathrm{H}), 4.36(\mathrm{dd}, J=$ 12.0, 3.0 Hz, 1H), $4.17(\mathrm{dd}, J=12.0,5.9 \mathrm{~Hz}, 1 \mathrm{H}), 4.01-3.94(\mathrm{~m}, 1 \mathrm{H}), 3.11-3.04(\mathrm{~m}, 1 \mathrm{H}), 2.18$ (s, 3H), 2.12 (s, 3H). ${ }^{13} \mathbf{C}$ NMR (101 MHz, $\left.\mathbf{C D C l}_{3}\right) \delta 170.8,170.1,140.9,138.5,133.3,130.8$, 129.6, 128.5, 127.9, 127.2, 88.7, 76.3, 73.2, 63.2, 50.3, 46.8, 29.7, 21.0, 20.8. HRMS (ESI+): $\mathrm{m} / \mathrm{z}$ calcd. For $\mathrm{C}_{18} \mathrm{H}_{19} \mathrm{ClNaO}_{5}(\mathrm{M}+\mathrm{Na})^{+}$: 373.0819; found 373.0799 . 


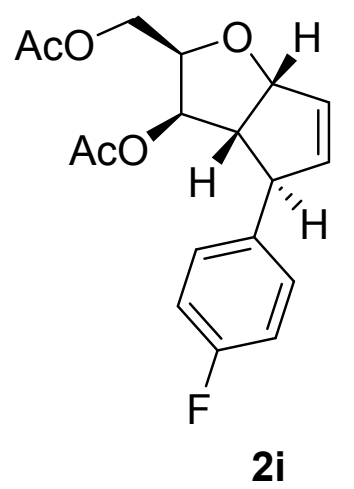

Prepared by general procedure A using tri- $O$-acetyl-D-galactal (100 mg, $0.36 \mathrm{mmol})$, 4-fluoro styrene $(43.5 \mu \mathrm{L}, 0.36 \mathrm{mmol})$ and $\mathrm{CF}_{3} \mathrm{SO}_{3} \mathrm{H}(63.5 \mu \mathrm{L}, 0.72 \mathrm{mmol})$. The reaction mixture was stirred at $0{ }^{\circ} \mathrm{C}$ for 30 minutes, and then quenched with triethylamine. The resulting mixture was extracted with $\mathrm{CH}_{2} \mathrm{Cl}_{2}$ and the combined organic layer was washed with brine, dried over $\mathrm{Na}_{2} \mathrm{SO}_{4}$ and concentrated. The residue was purified by flash column chromatography (Hexane: EtOAc; $97: 3$ ) to afford $\mathbf{2 i}$ as yellow oil in $43 \%$ yield, $51 \mathrm{mg}$.

${ }^{1}$ H NMR (400 MHz, CDCl $) \delta 7.07-7.02(\mathrm{~m}, 2 \mathrm{H}), 6.98(\mathrm{t}, J=8.4 \mathrm{~Hz}, 2 \mathrm{H}), 6.04(\mathrm{~s}, 1 \mathrm{H}), 5.93$ $(\mathrm{d}, J=4.9 \mathrm{~Hz}, 1 \mathrm{H}), 5.54(\mathrm{dd}, J=6.9,2.9 \mathrm{~Hz}, 1 \mathrm{H}), 5.30(\mathrm{~d}, J=7.9 \mathrm{~Hz}, 1 \mathrm{H}), 4.38-4.31(\mathrm{~m}, 1 \mathrm{H})$, $4.18-4.13(\mathrm{~m}, 1 \mathrm{H}), 3.78(\mathrm{~s}, 1 \mathrm{H}), 2.97$ (t, $J=6.0 \mathrm{~Hz}, 3 \mathrm{H}), 2.18(\mathrm{~s}, 3 \mathrm{H}), 2.09(\mathrm{~s}, 3 \mathrm{H}) .{ }^{13} \mathbf{C} \mathbf{N M R}$ (101 MHz, $\left.\mathbf{C D C l}_{3}\right) \delta 170.68,170.44,139.25,137.97,131.15,128.69,128.61,115.61,115.40$, 89.02, 79.84, 73.76, 62.59, 54.58, 50.10, 20.79, 20.77. HRMS (ESI+): $\mathrm{m} / \mathrm{z}$ calcd. for $\mathrm{C}_{18} \mathrm{H}_{19} \mathrm{FNaO}_{5}(\mathrm{M}+\mathrm{Na})^{+}: 357.1114$, found 357.1100 .

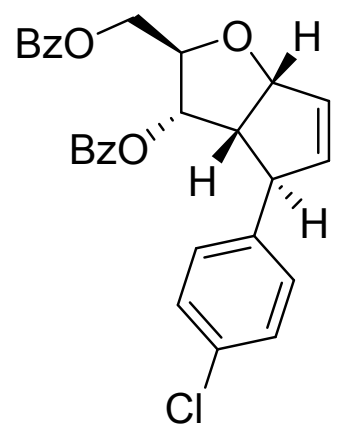

$2 \mathbf{j}$

Prepared by general procedure A using tri- $O$-benzoyl-D-glucal (100 mg, $0.22 \mathrm{mmol}$ ), 4-chloro styrene $(26.7 \mu \mathrm{L}, 0.22 \mathrm{mmol})$ and $\mathrm{CF}_{3} \mathrm{SO}_{3} \mathrm{H}(38.8 \mu \mathrm{L}, 0.44 \mathrm{mmol})$. The reaction mixture was stirred at $0{ }^{\circ} \mathrm{C}$ for 30 minutes, then quenched with triethylamine. The resulting mixture was extracted with $\mathrm{CH}_{2} \mathrm{Cl}_{2}$ and the combined organic layer was washed with brine, dried over $\mathrm{NaSO}_{4}$ and concentrated. The residue was purified by flash column chromatography (Hexane: EtOAc 97:3) to afford $\mathbf{2} \mathbf{j}$ as yellow oil in $53 \%$ yield, $54 \mathrm{mg}$. 
${ }^{1}$ H NMR (400 MHz, CDCl $) \delta 8.08(\mathrm{~d}, J=7.3 \mathrm{~Hz}, 2 \mathrm{H}), 8.03(\mathrm{~d}, J=7.4 \mathrm{~Hz}, 2 \mathrm{H}), 7.63(\mathrm{t}, J=$ $7.4 \mathrm{~Hz}, 1 \mathrm{H}), 7.54-7.46(\mathrm{~m}, 3 \mathrm{H}), 7.37(\mathrm{t}, J=7.7 \mathrm{~Hz}, 2 \mathrm{H}), 7.16(\mathrm{~d}, J=8.4 \mathrm{~Hz}, 2 \mathrm{H}), 6.89(\mathrm{~d}, J=$ $8.4 \mathrm{~Hz}, 2 \mathrm{H}), 6.04(\mathrm{dd}, J=5.5,2.0 \mathrm{~Hz}, 1 \mathrm{H}), 6.02-5.95(\mathrm{~m}, 1 \mathrm{H}), 5.52(\mathrm{~d}, J=6.8 \mathrm{~Hz}, 1 \mathrm{H}), 5.35(\mathrm{t}$, $J=8.5 \mathrm{~Hz}, 1 \mathrm{H}), 4.66(\mathrm{dd}, J=11.9,3.9 \mathrm{~Hz}, 1 \mathrm{H}), 4.50(\mathrm{dd}, J=11.9,5.4 \mathrm{~Hz}, 1 \mathrm{H}), 4.28-4.22(\mathrm{~m}$, 1H), 4.09 (s, 1H), 3.23 - 3.17 (m, 1H). ${ }^{13} \mathbf{C}$ NMR (126 MHz, $\left.\mathbf{C D C l}_{3}\right) \delta 166.35,165.67,142.12$, $138.76,133.62,133.13,132.40,131.13,129.76,129.73,129.34,128.75,128.69,128.57,128.35$, 88.54, 75.88, 74.79, 63.89, 51.69, 50.15. HRMS (ESI+): m/z calcd. for $\mathrm{C}_{28} \mathrm{H}_{24} \mathrm{ClO}_{5}(\mathrm{M}+\mathrm{H})^{+}$: 475.1312; found 475.1298 .

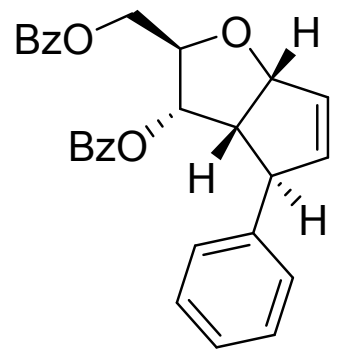

\section{$2 k$}

Prepared by general procedure A using tri-O-benzoyl-D-glucal (100 mg, $0.22 \mathrm{mmol})$, styrene (26 $\mu \mathrm{L}, 0.22 \mathrm{mmol})$ and $\mathrm{CF}_{3} \mathrm{SO}_{3} \mathrm{H}(38.8 \mu \mathrm{L}, 0.44 \mathrm{mmol})$. The reaction mixture was stirred at $0{ }^{\circ} \mathrm{C}$ for 30 minutes, and then quenched with triethylamine. The resulting mixture was extracted with $\mathrm{CH}_{2} \mathrm{Cl}_{2}$ and the combined organic layer was washed with brine, dried over $\mathrm{NaSO}_{4}$ and concentrated. The residue was purified by flash column chromatography (Hexane: EtOAc; 97:3) to afford $\mathbf{2 k}$ as yellow oil in $51 \%$ yield, $49 \mathrm{mg}$.

${ }^{1}$ H NMR (400 MHz, CDCl $\left.\mathbf{I}_{3}\right) \delta 8.12-8.08(\mathrm{~m}, 2 \mathrm{H}), 8.05-8.01(\mathrm{~m}, 2 \mathrm{H}), 7.62(\mathrm{t}, J=7.4 \mathrm{~Hz}$, 1H), $7.55-7.46(\mathrm{~m}, 4 \mathrm{H}), 7.38(\mathrm{t}, J=7.8 \mathrm{~Hz}, 2 \mathrm{H}), 7.23-7.17(\mathrm{~m}, 2 \mathrm{H}), 7.00-6.96(\mathrm{~m}, 2 \mathrm{H}), 6.09$ $(\mathrm{dd}, J=5.5,2.2 \mathrm{~Hz}, 1 \mathrm{H}), 6.02-5.98(\mathrm{~m}, 1 \mathrm{H}), 5.55(\mathrm{~d}, J=6.9 \mathrm{~Hz}, 1 \mathrm{H}), 5.40(\mathrm{t}, J=8.4 \mathrm{~Hz}, 1 \mathrm{H})$, $4.66(\mathrm{dd}, J=11.9,3.9 \mathrm{~Hz}, 1 \mathrm{H}), 4.51(\mathrm{dd}, J=11.9,5.4 \mathrm{~Hz}, 1 \mathrm{H}), 4.31-4.23(\mathrm{~m}, 1 \mathrm{H}), 4.17-4.11$ (m, 1H), 3.28 - 3.21 (m, 1H). ${ }^{13} \mathbf{C}$ NMR (126 MHz, $\left.\mathbf{C D C l}_{3}\right) \delta 166.37,165.68,143.61,139.22$, $133.51,133.09,130.66,129.77,129.76,129.50,128.66,128.63,128.34,127.23,126.67,88.74$, 76.05, 74.80, 63.99, 51.71, 50.77. HRMS (ESI+): $\mathrm{m} / \mathrm{z}$ calcd. for $\mathrm{C}_{28} \mathrm{H}_{25} \mathrm{O}_{5}(\mathrm{M}+\mathrm{H})^{+}: 441.1702$; found 441.1694 . 


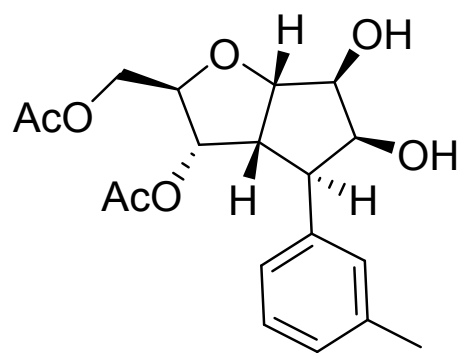

2aa

Prepared by using compound $8 \mathbf{a}(50 \mathrm{mg}, 0.15 \mathrm{mmol})$, in $t$-butanol: acetone: $\mathrm{H}_{2} \mathrm{O}(2: 1: 1)$, followed by the addition of $\mathrm{OsO}_{4}(7.6 \mathrm{~g}, 0.03 \mathrm{mmol})$ and $\mathrm{NMO}$ (catalytic) at rt. The reaction was stirred at rt untill complete consumption of starting material was observed by TLC. The solvent was evaporated in vacuo and the resulting reaction mixture was diluted with ethyl acetate washed with brine, dried over $\mathrm{NaSO}_{4}$ and concentrated. The residue was purified by flash column chromatography (Hexane: EtOAc; 9:1) to afford 2aa as colourless oil in 63\% yield, $35 \mathrm{mg}$.

${ }^{1}$ H NMR (400 MHz, CDCl $) \delta 7.26(\mathrm{~s}, 1 \mathrm{H}), 7.25-7.19(\mathrm{~m}, 1 \mathrm{H}), 7.07$ (d, J=7.5 Hz, 2H), 5.10 (t, $J=7.8 \mathrm{~Hz}, 1 \mathrm{H}), 4.64(\mathrm{dd}, J=7.9,4.0 \mathrm{~Hz}, 1 \mathrm{H}), 4.31(\mathrm{dd}, J=11.7,2.9 \mathrm{~Hz}, 1 \mathrm{H}), 4.24$ (s, 1H), 4.19 (t, $J=8.0 \mathrm{~Hz}, 2 \mathrm{H}), 4.14-4.09(\mathrm{~m}, 1 \mathrm{H}), 3.69-3.59(\mathrm{~m}, 1 \mathrm{H}), 3.30(\mathrm{dd}, J=10.9,2.7 \mathrm{~Hz}, 1 \mathrm{H}), 2.34$ (s, 3H), 2.09 (s, 3H), 1.52 (s, 3H). ${ }^{13} \mathbf{C}$ NMR (101 MHz, $\left.\mathbf{C D C l}_{3}\right) \delta$ 170.8, 169.9, 138.1, 137.2, $129.4,128.5,127.9,125.7,90.3,80.3,78.0,77.9,73.3,63.5,47.7,46.0,21.4,20.8,19.8$. HRMS (ESI+): $\mathrm{m} / \mathrm{z}$ calcd. for $\mathrm{C}_{19} \mathrm{H}_{24} \mathrm{NaO}_{7}(\mathrm{M}+\mathrm{Na})^{+}: 387.1420$, found 387.1403 .<smiles>CC(=O)OC[C@H]1O[C@H](O)C=C[C@@H]1OC(C)=O</smiles>

Prepared by general procedure A using tri- $O$-acetyl-D-glucal (100 mg, $0.36 \mathrm{mmol})$, styrene (42.5 $\mu \mathrm{L}, 0.36 \mathrm{mmol})$ and $\mathrm{CF}_{3} \mathrm{SO}_{3} \mathrm{H}(6.35 \mu \mathrm{L}, 0.07 \mathrm{mmol})$. The reaction mixture was stirred at $0{ }^{\circ} \mathrm{C}$ for 30 minutes, and then quenched with triethylamine. The resulting mixture was extracted with $\mathrm{CH}_{2} \mathrm{Cl}_{2}$ and the combined organic layer was washed with brine, dried over $\mathrm{Na}_{2} \mathrm{SO}_{4}$ and concentrated. The residue was purified by flash column chromatography (Hexane: EtOAc 97:3) to afford 6 as yellow oil in $60 \%$ yield, $50 \mathrm{mg}$.

${ }^{1}$ H NMR (400 MHz, CDCl $) \delta 5.92(\mathrm{~d}, J=10.2 \mathrm{~Hz}, 1 \mathrm{H}), 5.85-5.76(\mathrm{~m}, 1 \mathrm{H}), 5.42(\mathrm{~s}, 1 \mathrm{H}), 5.31$ $(\mathrm{dd}, J=9.7,1.2 \mathrm{~Hz}, 1 \mathrm{H}), 4.25-4.13(\mathrm{~m}, 2 \mathrm{H}), 4.12-4.04(\mathrm{~m}, 1 \mathrm{H}), 2.07(\mathrm{~s}, 6 \mathrm{H}) .{ }^{13} \mathrm{C}$ NMR (126 
MHz, $\left.\mathbf{C D C l}_{3}\right) \delta 170.6,170.1,129.6,127.3,90.5,67.3,65.1,62.8,20.9,20.7$. HRMS (ESI+): $\mathrm{m} / \mathrm{z}$ calcd. for $\mathrm{C}_{10} \mathrm{H}_{14} \mathrm{NaO}_{6}(\mathrm{M}+\mathrm{Na})^{+}:$253.0688, found 253.0671 .

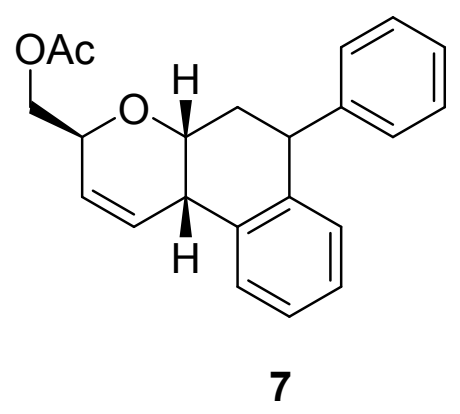

Prepared by general procedure B, using tri- $O$-acetyl-D-glucal (272 mg, $1.0 \mathrm{mmol})$, styrene (115.7 $\mu \mathrm{L}, 1.0 \mathrm{mmol})$ and $\mathrm{CF}_{3} \mathrm{SO}_{3} \mathrm{H}(26.4 \mu \mathrm{L}, 0.3 \mathrm{mmol})$. The reaction mixture was stirred at $0{ }^{\circ} \mathrm{C}$ for 30 minutes, and then quenched with triethylamine. The residue was purified by flash column chromatography (Hexane: EtOAc 49:1) to afford 7 as a colourless oil in 73\% yield, $244 \mathrm{mg}$.

${ }^{1}$ H NMR (400 MHz, CDCl M $\delta 7.37-7.32(\mathrm{~m}, 1 \mathrm{H}), 7.30$ (s, 1H), 7.27 (s, 1H), $7.21(\mathrm{~s}, 2 \mathrm{H}), 7.07$ $(\mathrm{dd}, J=13.2,7.3 \mathrm{~Hz}, 3 \mathrm{H}), 6.85(\mathrm{~d}, J=7.7 \mathrm{~Hz}, 1 \mathrm{H}), 6.30(\mathrm{dd}, J=10.3,3.0 \mathrm{~Hz}, 1 \mathrm{H}), 5.73(\mathrm{~d}, J=$ $10.3 \mathrm{~Hz}, 1 \mathrm{H}), 4.41-4.34(\mathrm{~m}, 3 \mathrm{H}), 4.30-4.25(\mathrm{~m}, 1 \mathrm{H}), 4.11$ (dd, $J=11.5,2.8 \mathrm{~Hz}, 1 \mathrm{H}), 3.59(\mathrm{~s}$, 1H), 2.45-2.39 (m, 1H), 2.10 (s, 3H), 2.05 (bd, $J=10.6 \mathrm{~Hz}, 1 \mathrm{H}) .{ }^{13} \mathbf{C}$ NMR (101 MHz, CDCl $) \delta$ 171.0, 146.1 138.3, 137.8, 131.1, 129.8, 128.8, 128.3, 128.3, 126.6, 126.2, 126.1, 124.6, 69.3, 66.0, 65.1, 42.2, 37.1, 35.1, 21.0. HRMS $(\mathrm{ESI}+): \mathrm{m} / \mathrm{z}$ calcd. for $\mathrm{C}_{22} \mathrm{H}_{22} \mathrm{NaO}_{3}(\mathrm{M}+\mathrm{Na})^{+}$: 357.1467 ; found 357.1459 .<smiles>CC(=O)OC[C@H]1C=C[C@H]2c3ccccc3C(c3ccccc3Br)C[C@H]2O1</smiles>

$7 a$

Prepared by general procedure B using tri- $O$-acetyl-D-glucal (100 mg, $0.36 \mathrm{mmol})$, 4bromostyrene $(47.05 \mu \mathrm{L}, 0.36 \mathrm{mmol})$ and $\mathrm{CF}_{3} \mathrm{SO}_{3} \mathrm{H}(9.5 \mu \mathrm{L}, 0.1 \mathrm{mmol})$. The residue was purified by flash column chromatography (Hexane: EtOAc 49:1) to afford 7a as a colourless oil in $56 \%$ yield, $83 \mathrm{mg}$.

${ }^{1}$ H NMR (400 MHz, $\left.\mathbf{C D C l}_{3}\right) \delta 7.59(\mathrm{dd}, J=7.9,1.3 \mathrm{~Hz}, 1 \mathrm{H}), 7.34(\mathrm{~d}, J=7.7 \mathrm{~Hz}, 1 \mathrm{H}), 7.25(\mathrm{~d}, J$ $=7.2 \mathrm{~Hz}, 1 \mathrm{H}), 7.15-7.09(\mathrm{~m}, 2 \mathrm{H}), 7.05(\mathrm{td}, J=7.6,1.8 \mathrm{~Hz}, 1 \mathrm{H}), 6.87(\mathrm{~d}, J=7.7 \mathrm{~Hz}, 1 \mathrm{H}), 6.69$ 
$(\mathrm{d}, J=7.6 \mathrm{~Hz}, 1 \mathrm{H}), 6.21-6.15(\mathrm{~m}, 1 \mathrm{H}), 5.70(\mathrm{dt}, J=10.3,2.3 \mathrm{~Hz}, 1 \mathrm{H}), 4.84(\mathrm{t}, J=5.8 \mathrm{~Hz}, 1 \mathrm{H})$, 4.37 (ddd, $J=9.5,5.1,2.9 \mathrm{~Hz}, 2 \mathrm{H}), 4.21(\mathrm{dd}, J=11.5,7.2 \mathrm{~Hz}, 1 \mathrm{H}), 4.15$ (dd, $J=11.5,3.9 \mathrm{~Hz}$, 1H), $3.70(\mathrm{~s}, 1 \mathrm{H}), 2.44$ (ddd, $J=13.1,9.5,5.9 \mathrm{~Hz}, 1 \mathrm{H}), 2.14$ (d, $J=2.3 \mathrm{~Hz}, 1 \mathrm{H}), 2.11$ (s, 3H). ${ }^{13}$ C NMR (101 MHz, $\left.\mathbf{C D C l}_{3}\right) \delta$ 171.0, 144.9, 138.6, 137.3, 133.0, 131.2, 130.8, 129.8, 128.7, $127.8,127.2$, 127.0, 126.5, 124.7, 124.4, 68.4, 66.5, 65.7, 42.7, 37.5, 31.4, 21.0. HRMS (ESI+): $\mathrm{m} / \mathrm{z}$ calcd. for $\mathrm{C}_{22} \mathrm{H}_{21} \mathrm{BrNaO}_{3}(\mathrm{M}+\mathrm{Na})^{+}$: 435.0572; found 435.0569 .<smiles>CC(=O)OC[C@H]1C=C[C@H]2c3cccc(Br)c3C(c3ccccc3)C[C@H]2O1</smiles>

Prepared by general procedure B using tri- $O$-acetyl-D-glucal (100 mg, $0.36 \mathrm{mmol})$, 4bromostyrene $(47.05 \mu \mathrm{L}, 0.36 \mathrm{mmol})$ and $\mathrm{CF}_{3} \mathrm{SO}_{3} \mathrm{H}(9.5 \mu \mathrm{L}, 0.1 \mathrm{mmol})$. The residue was purified by flash column chromatography (Hexane: EtOAc 49:1) to afford 7a` as a colourless oil in $21 \%$ yield, $31 \mathrm{mg}$.

${ }^{1}$ H NMR (400 MHz, CDCl $) \delta 7.47(\mathrm{~d}, J=7.1 \mathrm{~Hz}, 1 \mathrm{H}), 7.32(\mathrm{~d}, J=7.7 \mathrm{~Hz}, 1 \mathrm{H}), 7.22(\mathrm{~d}, J=$ $7.7 \mathrm{~Hz}, 2 \mathrm{H}), 7.18$ (dd, $J=7.5,3.2 \mathrm{~Hz}, 2 \mathrm{H}), 6.90$ (d, $J=7.1 \mathrm{~Hz}, 2 \mathrm{H}), 5.93-5.87$ (m, 1H), 5.69$5.61(\mathrm{~m}, 1 \mathrm{H}), 4.63(\mathrm{~d}, J=3.4 \mathrm{~Hz}, 1 \mathrm{H}), 4.42-4.36(\mathrm{~m}, 1 \mathrm{H}), 4.34-4.29(\mathrm{~m}, 1 \mathrm{H}), 4.21(\mathrm{dd}, J=11.4$, $3.5 \mathrm{~Hz}, 1 \mathrm{H}), 4.04(\mathrm{dd}, J=11.4,7.2 \mathrm{~Hz}, 1 \mathrm{H}), 3.78$ (d, $J=3.0 \mathrm{~Hz}, 1 \mathrm{H}), 2.53-2.46(\mathrm{~m}, 1 \mathrm{H}), 2.17-$ 2.12 (m, 1H), 2.09 (s, 3H). ${ }^{13} \mathbf{C}$ NMR (101 MHz, $\left.\mathbf{C D C l}_{3}\right) \delta 171.0,143.8,141.8,136.7,131.5$, 131.2 , 128.7, 128.5, 128.2, 128.1, 126.2, 126.0, 124.3, 67.1, 66.8, 66.3, 45.4, 38.1, 31.9, 20.9. HRMS (ESI+): $\mathrm{m} / \mathrm{z}$ calcd. for $\mathrm{C}_{22} \mathrm{H}_{21} \mathrm{BrNaO}_{3}(\mathrm{M}+\mathrm{Na})^{+}$: 435.0572; found 435.0587.<smiles>CC(=O)C[C@H]1C=C[C@H]2c3ccccc3C(c3ccccc3Cl)C[C@H]2O1</smiles>

7b

Prepared by general procedure B using tri- $O$-acetyl-D-glucal (100 mg, $0.36 \mathrm{mmol})$, 2chlorostyrene $(45.74 \mu \mathrm{L}, 0.36 \mathrm{mmol})$ and $\mathrm{CF}_{3} \mathrm{SO}_{3} \mathrm{H}(9.5 \mu \mathrm{L}, 0.1 \mathrm{mmol})$. The residue was purified by flash column chromatography (Hexane: EtOAc 49:1) to afford $\mathbf{7 b}$ as a colourless oil in $67 \%$ yield, $89 \mathrm{mg}$. 
${ }^{1}$ H NMR (400 MHz, $\left.\mathbf{C D C l}_{3}\right) \delta 7.39(\mathrm{dd}, J=7.8,1.3 \mathrm{~Hz}, 1 \mathrm{H}), 7.34(\mathrm{~d}, J=7.7 \mathrm{~Hz}, 1 \mathrm{H}), 7.24(\mathrm{~d}, J$ $=7.8 \mathrm{~Hz}, 1 \mathrm{H}), 7.16-7.06(\mathrm{~m}, 3 \mathrm{H}), 6.87(\mathrm{~d}, J=7.7 \mathrm{~Hz}, 1 \mathrm{H}), 6.70(\mathrm{dd}, J=7.6,1.4 \mathrm{~Hz}, 1 \mathrm{H}), 6.27-$ $6.07(\mathrm{~m}, 1 \mathrm{H}), 5.70(\mathrm{dt}, J=10.3,2.3 \mathrm{~Hz}, 1 \mathrm{H}), 4.86(\mathrm{t}, J=5.9 \mathrm{~Hz}, 1 \mathrm{H}), 4.44-4.32(\mathrm{~m}, 2 \mathrm{H}), 4.38$ $4.13(\mathrm{~m}, 2 \mathrm{H}), 3.69$ (s, 1H), 2.47-2.40 (m, 1H), 2.18-2.12 (m, 1H), $2.11(\mathrm{~s}, 3 \mathrm{H}) .{ }^{13} \mathbf{C}$ NMR (101 MHz, $\left.\mathbf{C D C l}_{3}\right) \delta 171.0,143.3,138.6,137.3,134.0,131.2,130.7,129.7,129.7,128.7,127.5$, 126.9, 126.6, 126.5, 124.4, 68.5, 66.5, 65.6, 40.1, 37.4, 31.2, 21.0. HRMS (ESI+): m/z calcd. for $\mathrm{C}_{22} \mathrm{H}_{21} \mathrm{ClNaO}_{3}(\mathrm{M}+\mathrm{Na})^{+}$: 391.1077; found 391.1093.<smiles>CC(=O)OC[C@H]1C=C[C@H]2c3cccc(Br)c3C(c3ccc(C)cc3)C[C@H]2O1</smiles>

Prepared by general procedure B using tri- $O$-acetyl-D-glucal $(100 \mathrm{mg}, 0.36 \mathrm{mmol})$, 4bromostyrene $(47.05 \mu \mathrm{L}, 0.36 \mathrm{mmol})$ and $\mathrm{CF}_{3} \mathrm{SO}_{3} \mathrm{H}(9.5 \mu \mathrm{L}, 0.1 \mathrm{mmol})$ in toluene. The residue was purified by flash column chromatography (Hexane: EtOAc 49:1) to afford 7c as a colourless oil in $75 \%$ yield, $115 \mathrm{mg}$.

${ }^{1}$ H NMR (400 MHz, $\left.\mathbf{C D C l}_{3}\right) \delta 7.48-7.42(\mathrm{~m}, 1 \mathrm{H}), 7.31$ (d, $\left.J=7.7 \mathrm{~Hz}, 1 \mathrm{H}\right), 7.18(\mathrm{t}, J=7.8 \mathrm{~Hz}$, 1H), $7.04(\mathrm{~d}, J=7.8 \mathrm{~Hz}, 2 \mathrm{H}), 6.78(\mathrm{~d}, J=8.0 \mathrm{~Hz}, 2 \mathrm{H}), 5.90(\mathrm{dd}, J=10.4,0.9 \mathrm{~Hz}, 1 \mathrm{H}), 5.64$ (ddd, $J=10.4,2.7,1.7 \mathrm{~Hz}, 1 \mathrm{H}), 4.60$ (d, $J=3.5 \mathrm{~Hz}, 1 \mathrm{H}), 4.38$ (ddd, $J=12.2,5.8,3.6 \mathrm{~Hz}, 1 \mathrm{H})$, 4.33-4.28 (m, 1H), $4.20(\mathrm{dd}, J=11.4,3.5 \mathrm{~Hz}, 1 \mathrm{H}), 4.05(\mathrm{dd}, J=11.4,7.3 \mathrm{~Hz}, 1 \mathrm{H}), 3.79-3.73$ (m, 1H), 2.48 (ddd, $J=17.8,12.0,5.8 \mathrm{~Hz}, 1 \mathrm{H}), 2.29$ (s, 3H), 2.13 (dd, $J=8.7,6.1 \mathrm{~Hz}, 1 \mathrm{H}), 2.09$ (s, 3H). ${ }^{13} \mathbf{C}$ NMR (101 MHz, $\left.\mathbf{C D C l}_{3}\right) \delta$ 171.0, 141.8, 140.7, 137.0, 135.6, 131.5, 131.2, 128.9, 128.6, 128.5, 128.0, 126.0, 124.3, 67.1, 66.8, 66.3, 45.0, 38.1, 31.9, 21.0, 20.9. HRMS (ESI+): $\mathrm{m} / \mathrm{z}$ calcd. for $\mathrm{C}_{23} \mathrm{H}_{23} \mathrm{BrNaO}_{3}(\mathrm{M}+\mathrm{Na})^{+}$: 449.0728; found 449.0739.

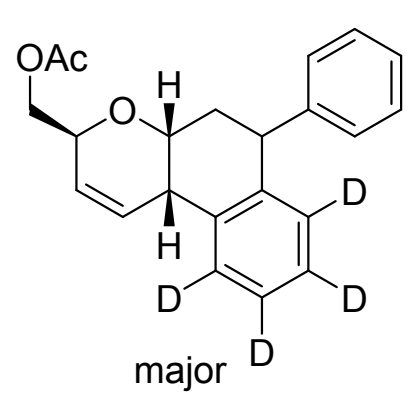<smiles>[2H]c1c([2H])c([2H])c(C2C[C@H]3O[C@H](COC(C)=O)C=C[C@H]3c3ccccc32)c([2H])c1[2H]</smiles> 
Prepared by general procedure $\mathbf{B}$ using tri- $O$-acetyl-D-glucal $(50 \mathrm{mg}, 0.18 \mathrm{mmol})$, styrene (20.8 $\mu \mathrm{L}, 0.18 \mathrm{mmol})$ and $\mathrm{CF}_{3} \mathrm{SO}_{3} \mathrm{H}(4.8 \mu \mathrm{L}, 0.05 \mathrm{mmol})$ in deuterated benzene- $\mathrm{d}_{6}$. Isolated by flash column chromatography (Hexane: EtOAc 49:1) to afford 7d as a mixture of regioisomers in the ratio of 2.5:1 as a colourless oil in 33\% yield, $20 \mathrm{mg}$.

${ }^{1}$ H NMR (400 MHz, $\mathbf{C D C l}_{3}$ ) $\delta$ 7.41-7.27 (m, 4H; 2H-major isomer, 2H-minor isomer), 7.277.21 (m, 2H; 1H-major isomer , 1H-minor isomer), 7.11 (dd, $J=14.2,7.1 \mathrm{~Hz}, 2 \mathrm{H}), 6.89$ (d, $J=$ $7.8 \mathrm{~Hz}, 1 \mathrm{H}$; minor isomer), 6.34 (ddd, $J=10.4,4.4,2.2 \mathrm{~Hz}, 1 \mathrm{H}), 5.77$ (dt, $J=10.3,2.3 \mathrm{~Hz}, 1 \mathrm{H}$ ), 4.48-4.36 (m, 4H; 3H-major, 1H-minor), 4.14 (dd, $J=11.5,3.5 \mathrm{~Hz}, 1 \mathrm{H}), 3.63(\mathrm{~s}, 1 \mathrm{H}), 2.49-$ $2.42(\mathrm{~m}, 1 \mathrm{H}), 2.14(\mathrm{~s}, 3 \mathrm{H}), 2.10(\mathrm{dd}, J=4.6,2.8 \mathrm{~Hz}, 1 \mathrm{H}) .{ }^{13} \mathbf{C} \mathbf{N M R}\left(\mathbf{1 0 1} \mathbf{M H z}, \mathbf{C D C l}_{3}\right) \delta 170.9$, $146.1,138.4$, 137.8, 131.0, 129.8, 128.8, 128.3, 128.3, 126.6, 126.2, 126.1, 124.6, 69.3, 69.3 (minor isomer), 66.0 (minor isomer), 66.0, 65.1 (minor isomer), 65.1, 42.1, 37.2 (minor isomer), 37.1, 35.2, 35.2 (minor isomer), 20.9 (s). HRMS (ESI+): $\mathrm{m} / \mathrm{z}$ calcd. for major isomer $\mathrm{C}_{22} \mathrm{H}_{18} \mathrm{D}_{4} \mathrm{NaO}_{3}(\mathrm{M}+\mathrm{Na})^{+}$: 361.1718; found 361.1739. HRMS $(\mathrm{ESI}+): \mathrm{m} / \mathrm{z}$ calcd. for minor isomer $\mathrm{C}_{22} \mathrm{H}_{17} \mathrm{D}_{5} \mathrm{NaO}_{3}(\mathrm{M}+\mathrm{Na})^{+}$: 362.1780; found 361.1799.

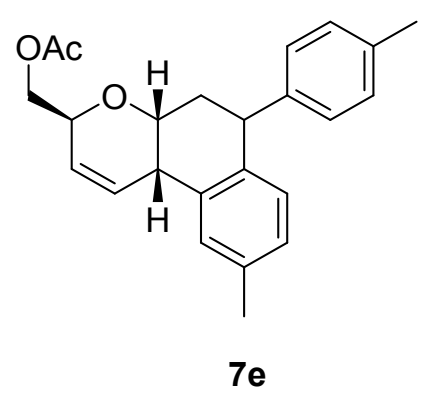

Prepared by general procedure B using tri- $O$-acetyl-D-glucal $(100 \mathrm{mg}, 0.36 \mathrm{mmol})$, 4methylstyrene $(47.73 \mu \mathrm{L}, 0.36 \mathrm{mmol})$ and $\mathrm{CF}_{3} \mathrm{SO}_{3} \mathrm{H}(9.5 \mu \mathrm{L}, 0.1 \mathrm{mmol})$ in toluene. The residue was purified by flash column chromatography (Hexane: EtOAc 49:1) to afford 7e as a colourless oil in $61 \%$ yield, $79 \mathrm{mg}$.

${ }^{1}$ H NMR (400 MHz, $\left.\mathbf{C D C l}_{3}\right) \delta 7.11-7.06(\mathrm{~m}, 3 \mathrm{H}), 6.98(\mathrm{~d}, J=7.6 \mathrm{~Hz}, 2 \mathrm{H}), 6.87(\mathrm{~d}, J=7.8 \mathrm{~Hz}$, 1H), $6.74(\mathrm{~d}, J=7.9 \mathrm{~Hz}, 1 \mathrm{H}), 6.30$ (d, $J=10.2 \mathrm{~Hz}, 1 \mathrm{H}), 5.72$ (d, $J=10.2 \mathrm{~Hz}, 1 \mathrm{H}), 4.35$ (s, 2H), $4.27(\mathrm{dd}, J=11.6,7.5 \mathrm{~Hz}, 2 \mathrm{H}), 4.12-4.07(\mathrm{~m}, 1 \mathrm{H}), 3.53(\mathrm{~s}, 1 \mathrm{H}), 2.37$ (d, $J=6.5 \mathrm{~Hz}, 1 \mathrm{H}), 2.31$ (s, 6H), 2.09 (s, 3H), 2.01 (d, $J=10.5 \mathrm{~Hz}, 1 \mathrm{H}) .{ }^{\mathbf{1 3}} \mathbf{C}$ NMR (101 MHz, $\left.\mathbf{C D C l}_{3}\right) \delta 171.0,143.3$, 137.5, 136.0, 135.6, 135.5, 131.2, 129.7, 129.0, 128.7, 128.7, 127.1, 124.5, 69.3, 66.1, 65.1, 41.4, 37.1, 35.3, 21.1, 21.0. HRMS (ESI+): $\mathrm{m} / \mathrm{z}$ calcd. for $\mathrm{C}_{24} \mathrm{H}_{26} \mathrm{NaO}_{3}(\mathrm{M}+\mathrm{Na})^{+}$: 385.1780; found 385.1769 . 
<smiles>C[C@H]1C=C[C@H]2c3ccccc3C(c3ccccc3)C[C@@H]2O1</smiles>

$7 f$

Prepared by general procedure B using di- $O$-acetyl-L-Rhamnal (100 mg, $0.46 \mathrm{mmol})$, styrene $(53.15 \mu \mathrm{L}, 0.46 \mathrm{mmol})$ and $\mathrm{CF}_{3} \mathrm{SO}_{3} \mathrm{H}(12.1 \mu \mathrm{L}, 0.13 \mathrm{mmol})$ in benzene. Isolated by flash column chromatography (Hexane: EtOAc 49:1) to afford $7 \mathbf{f}$ as a colourless oil in 47\% yield, $59 \mathrm{mg}$.

${ }^{1}$ H NMR (400 MHz, $\left.\mathbf{C D C l}_{3}\right) \delta 7.33(\mathrm{dd}, J=14.9,7.4 \mathrm{~Hz}, 2 \mathrm{H}), 7.29(\mathrm{~s}, 1 \mathrm{H}), 7.23(\mathrm{td}, J=7.3$, $4.2 \mathrm{~Hz}, 2 \mathrm{H}), 7.14-7.06(\mathrm{~m}, 3 \mathrm{H}), 6.88$ (d, $J=7.8 \mathrm{~Hz}, 1 \mathrm{H}), 6.20-6.11(\mathrm{~m}, 1 \mathrm{H}), 5.78$ (dt, $J=10.2$, $2.3 \mathrm{~Hz}, 1 \mathrm{H}), 4.44-4.38(\mathrm{~m}, 1 \mathrm{H}), 4.37-4.31(\mathrm{~m}, 2 \mathrm{H}), 3.57(\mathrm{~s}, 1 \mathrm{H}), 2.51-2.44(\mathrm{~m}, 1 \mathrm{H}), 2.12-2.06$ $(\mathrm{m}, 1 \mathrm{H}), 1.32(\mathrm{~d}, J=6.7 \mathrm{~Hz}, 3 \mathrm{H}) .{ }^{13} \mathbf{C} \mathbf{N M R}\left(\mathbf{1 0 1} \mathbf{M H z}, \mathbf{C D C l}_{3}\right) \delta 146.3,138.4,138.3,130.6$, $129.8,128.8,128.4,128.3,127.5,126.5,126.1,125.9$, 66.7, 65.2, 42.4, 37.2, 35.4, 20.2. HRMS (ESI+): m/z calcd. for $\mathrm{C}_{20} \mathrm{H}_{20} \mathrm{NaO}(\mathrm{M}+\mathrm{Na})^{+}$: 299.1412; found 299.1479.<smiles>CC(=O)OC[C@H]1C=C[C@H]2c3cccc(Cl)c3C(c3ccc(C)cc3)C[C@H]2O1</smiles>

Prepared by general procedure B using tri- $O$-acetyl-D-glucal $(100 \mathrm{mg}, 0.36 \mathrm{mmol})$, 2chlorostyrene $(45.74 \mu \mathrm{L}, 0.36 \mathrm{mmol})$ and $\mathrm{CF}_{3} \mathrm{SO}_{3} \mathrm{H}(9.5 \mu \mathrm{L}, 0.1 \mathrm{mmol})$ in toluene. Isolated by flash column chromatography (Hexane: EtOAc 49:1) to afford $\mathbf{7 g}$ as a colourless oil in $68 \%$ yield, $93 \mathrm{mg}$.

${ }^{1}$ H NMR (400 MHz, $\left.\mathbf{C D C l}_{3}\right) \delta 7.26(\mathrm{~d}, J=0.6 \mathrm{~Hz}, 3 \mathrm{H}), 7.03(\mathrm{~d}, J=7.7 \mathrm{~Hz}, 2 \mathrm{H}), 6.78(\mathrm{~d}, J=$ $7.7 \mathrm{~Hz}, 2 \mathrm{H}), 5.90$ (d, $J=10.1 \mathrm{~Hz}, 1 \mathrm{H}), 5.64$ (d, $J=10.3 \mathrm{~Hz}, 1 \mathrm{H}), 4.61$ (d, $J=4.2 \mathrm{~Hz}, 1 \mathrm{H}), 4.42-$ $4.35(\mathrm{~m}, 1 \mathrm{H}), 4.31(\mathrm{~s}, 1 \mathrm{H}), 4.19(\mathrm{dd}, J=11.5,3.2 \mathrm{~Hz}, 1 \mathrm{H}), 4.04(\mathrm{dd}, J=11.3,7.2 \mathrm{~Hz}, 1 \mathrm{H}), 3.76$ (s, 1H), 2.51-2.41 (m, 1H), $2.28(\mathrm{~s}, 3 \mathrm{H}), 2.13(\mathrm{~d}, J=8.6 \mathrm{~Hz}, 1 \mathrm{H}), 2.09(\mathrm{~s}, 3 \mathrm{H}) .{ }^{13} \mathbf{C}$ NMR (101 MHz, $\mathbf{C D C l}_{3} \delta 171.0,141.6,140.8,135.6,135.4,134.8,131.2,128.9,128.2,128.0,127.7$, 124.3, 67.0, 66.8, 66.3, 42.5, 38.0, 31.8, 20.9, 20.9. HRMS (ESI+): $\mathrm{m} / \mathrm{z}$ calcd. for $\mathrm{C}_{23} \mathrm{H}_{23} \mathrm{ClNaO}_{3}$ $(\mathrm{M}+\mathrm{Na})^{+}:$405.1233; found 405.1259 . 

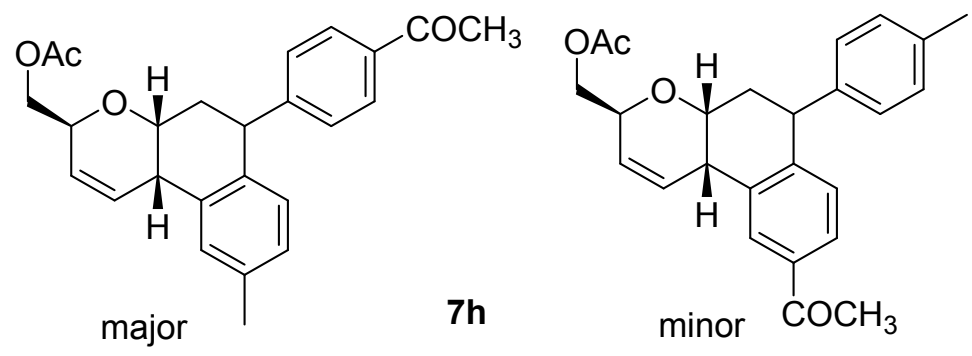

Prepared by general procedure B using tri- $O$-acetyl-D-glucal (100 mg, $0.36 \mathrm{mmol})$, 4acetoxystyrene $(55.01 \mu \mathrm{L}, 0.36 \mathrm{mmol})$ and $\mathrm{CF}_{3} \mathrm{SO}_{3} \mathrm{H}(9.5 \mu \mathrm{L}, 0.1 \mathrm{mmol})$ in toluene. Isolated by flash column chromatography (Hexane: EtOAc 48:2) to afford $\mathbf{7 h}$ as a mixture of regioisomers in the ratio of 1.5:1 as a colourless oil in $61 \%$ yield, $86 \mathrm{mg}$.

${ }^{1}$ H NMR (400 MHz, $\left.\mathbf{C D C l}_{3}\right) \delta$ 7.26-7.07 (m, 3H), 7.03-6.97 (m, 2H), 6.93-6.81 (m, 1H), 6.79$6.72(\mathrm{~m}, 1 \mathrm{H}), 6.30(\mathrm{~d}, J=7.9 \mathrm{~Hz}, 1 \mathrm{H}), 5.76(\mathrm{dd}, J=29.8,18.8 \mathrm{~Hz}, 1 \mathrm{H}), 4.39-4.24(\mathrm{~m}, 4 \mathrm{H})$, 4.13-4.05 (m, 1H), $3.53(\mathrm{~s}, 1 \mathrm{H}), 2.38(\mathrm{~d}, J=5.9 \mathrm{~Hz}, 1 \mathrm{H}), 2.32(\mathrm{~s}, 3 \mathrm{H}), 2.28(\mathrm{~s}, 3 \mathrm{H}), 2.10(\mathrm{~s}, 3 \mathrm{H})$, $2.01(\mathrm{dd}, J=12.0,7.7 \mathrm{~Hz}, 1 \mathrm{H}) .{ }^{13} \mathbf{C}$ NMR (101 $\left.\mathbf{~ M H z}, \mathbf{C D C l}_{3}\right)$ of major isomer $\delta 171.0,169.5$, 149.0, 143.8, 137.5, 136.2, 135.0, 131.1, 129.7, 129.1, 128.7, 127.1, 121.3, 120.7, 69.4, 65.9, 65.1, 41.3, 37.0, 35.4, 21.1, 21.1, 21.0. ${ }^{\mathbf{1 3}} \mathbf{C}$ NMR (101 $\mathbf{M H z}, \mathbf{C D C l}_{\mathbf{3}}$ ) of minor isomer $\delta$ 171.0, $169.5,149.1,142.8,138.9,136.3,135.9,130.7,130.3,129.7,128.8,125.1,124.5,119.3,69.7$, 65.6, 64.8, 40.9, 35.7. HRMS (ESI+): $\mathrm{m} / \mathrm{z}$ calcd. for $\mathrm{C}_{25} \mathrm{H}_{26} \mathrm{NaO}_{4}(\mathrm{M}+\mathrm{Na})^{+}$: 413.1729; found 413.1789 .

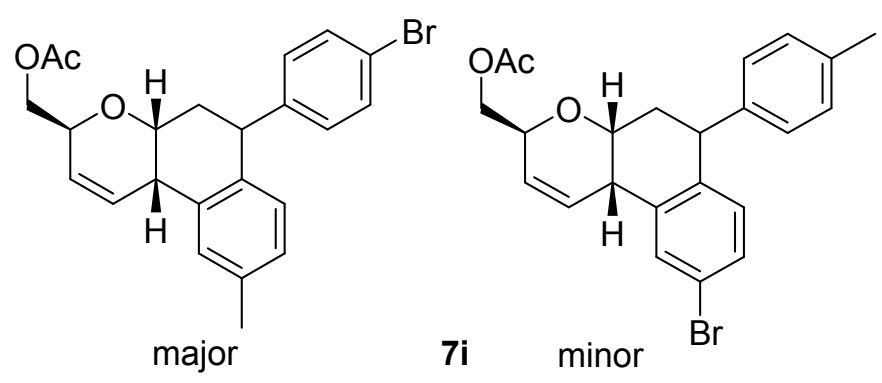

Prepared by general procedure B using tri- $O$-acetyl-D-glucal $(100 \mathrm{mg}, 0.36 \mathrm{mmol})$, 4bromostyrene $(47.05 \mu \mathrm{L}, 0.36 \mathrm{mmol})$ and $\mathrm{CF}_{3} \mathrm{SO}_{3} \mathrm{H}(9.5 \mu \mathrm{L}, 0.1 \mathrm{mmol})$ in toluene. Isolated by flash column chromatography (Hexane: EtOAc 49:1) to afford 7i as a mixture of regioisomers in the ratio of 1.01:1 as a colourless oil in $63 \%$ yield, $96 \mathrm{mg}$.

${ }^{1}$ H NMR (400 MHz, $\left.\mathbf{C D C l}_{3}\right) \delta 7.45-7.33(\mathrm{~m}, 2 \mathrm{H}), 7.17-7.09(\mathrm{~m}, 2 \mathrm{H}), 6.98(\mathrm{~d}, J=8.3 \mathrm{~Hz}, 2 \mathrm{H})$, $6.89(\mathrm{~d}, J=7.8 \mathrm{~Hz}, 1 \mathrm{H}$, minor isomer), $6.70(\mathrm{t}, J=8.6 \mathrm{~Hz}, 1 \mathrm{H}), 6.38-6.25(\mathrm{~m}, 1 \mathrm{H}), 5.82-5.68$ $(\mathrm{m}, 1 \mathrm{H}), 4.39-4.25(\mathrm{~m}, 4 \mathrm{H}), 4.13-4.03(\mathrm{~m}, 1 \mathrm{H}), 3.52(\mathrm{~s}, 1 \mathrm{H}), 2.37(\mathrm{t}, J=6.4 \mathrm{~Hz}, 1 \mathrm{H}), 2.32(\mathrm{~d}$, 
$J=4.3 \mathrm{~Hz}, 3 \mathrm{H}), 2.11(\mathrm{~s}, 3 \mathrm{H}), 2.02-1.94(\mathrm{~m}, 1 \mathrm{H}) .{ }^{13} \mathbf{C}$ NMR (126 MHz, CDCl $\left.\mathbf{3}\right) \delta 171.0$, 145.4, 139.9, 136.3, 134.8, 131.4, 130.6, 130.2, 129.2, 128.8, 128.7, 124.6, 120.0, 69.6, 65.7, 64.9, 41.2, 36.8, 35.5, 21.1, 21.0. ${ }^{13} \mathbf{C}$ NMR (126 $\mathbf{~ M H z , ~} \mathbf{C D C l}_{3}$ ) for minor isomer $\delta 170.9,142.5$, $137.9,137.5,136.0,131.6,131.0,129.9,129.6,129.1,127.2,125.4,120.2,69.9,65.4,64.7,40.9$, 36.9, 35.8, 21.0, 21.0. HRMS (ESI+): m/z calcd. for $\mathrm{C}_{23} \mathrm{H}_{23} \mathrm{BrNaO}_{3}(\mathrm{M}+\mathrm{Na})^{+}$: 449.0728; found 449.0717 .

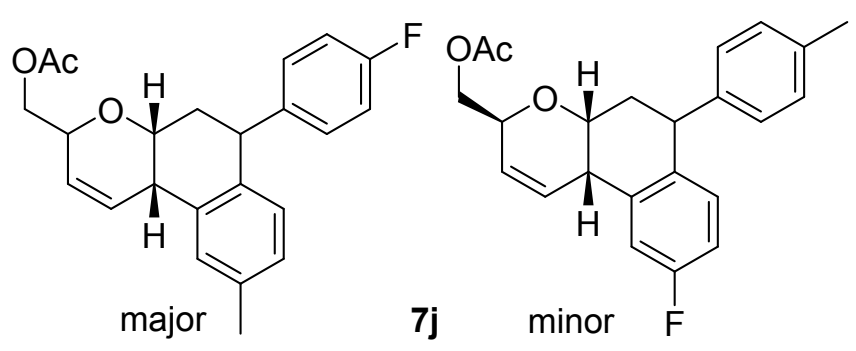

Prepared by general procedure B using tri- $O$-acetyl-D-glucal (100 mg, $0.36 \mathrm{mmol})$, 4fluorostyrene $(43.05 \mu \mathrm{L}, 0.36 \mathrm{mmol})$ and $\mathrm{CF}_{3} \mathrm{SO}_{3} \mathrm{H}(9.5 \mu \mathrm{L}, 0.1 \mathrm{mmol})$ in toluene. Isolated by flash column chromatography (Hexane: EtOAc 49:1) to afford $\mathbf{7 j}$ as a mixture of regioisomers in the ratio of $1.16: 1$ as a colourless oil in $59 \%$ yield, $78 \mathrm{mg}$.

${ }^{1}$ H NMR (400 MHz, CDCl $) \delta$ 7.16-7.07 (m, 3H), 7.05-6.98 (m, 2H), 6.95-6.73 (m, 2H), 6.39$6.31(\mathrm{~m}, 1 \mathrm{H}), 5.86-5.72(\mathrm{~m}, 1 \mathrm{H}), 4.44-4.29(\mathrm{~m}, 4 \mathrm{H}), 4.15-4.10(\mathrm{~m}, 1 \mathrm{H}), 3.58(\mathrm{~d}, J=4.7 \mathrm{~Hz}$, $1 \mathrm{H}), 3.45(\mathrm{~d}, J=5.2 \mathrm{~Hz}, 1 \mathrm{H}), 2.43(\mathrm{dd}, J=12.7,5.9 \mathrm{~Hz}, 1 \mathrm{H}), 2.36(\mathrm{~d}, J=3.3 \mathrm{~Hz}, 3 \mathrm{H}), 2.14(\mathrm{~s}$, $3 \mathrm{H}), 2.05(\mathrm{dd}, J=7.7,5.1 \mathrm{~Hz}, 1 \mathrm{H}) .{ }^{13} \mathbf{C}$ NMR (101 MHz, $\left.\mathbf{C D C l}_{3}\right) \delta 170.9,162.6(\mathrm{~d}, J=4.2 \mathrm{~Hz})$, $142.9,142.0$ (d, $J=3.2 \mathrm{~Hz}), 136.2,135.2,130.25$ (d, $J=5.8 \mathrm{~Hz}), 130.1,129.1,128.64,127.1$, $115.1(\mathrm{~d}, J=21.1 \mathrm{~Hz}), 114.3(\mathrm{~d}, J=21.0 \mathrm{~Hz}), 69.5,65.9,65.0,41.0,37.0,35.5,21.0,20.9 .{ }^{13} \mathbf{C}$ NMR (101 MHz, $\left.\mathbf{C D C l}_{3}\right)$ of minor isomer $\delta 170.9,160.2(\mathrm{~d}, J=4.0 \mathrm{~Hz}), 144.6,139.6(\mathrm{~d}, J=$ $6.8 \mathrm{~Hz}), 137.5,135.9,131.3(\mathrm{~d}, J=8.0 \mathrm{~Hz}), 131.0,129.6,128.8,125.3,124.6,113.3(\mathrm{~d}, J=21.1$ $\mathrm{Hz}), 69.8,65.62,64.8,40.8,37.1,35.7$. HRMS (ESI+): $\mathrm{m} / \mathrm{z}$ calcd. for $\mathrm{C}_{23} \mathrm{H}_{23} \mathrm{FNaO}_{3}(\mathrm{M}+\mathrm{Na})^{+}$: 389.1529; found 389.1568 .

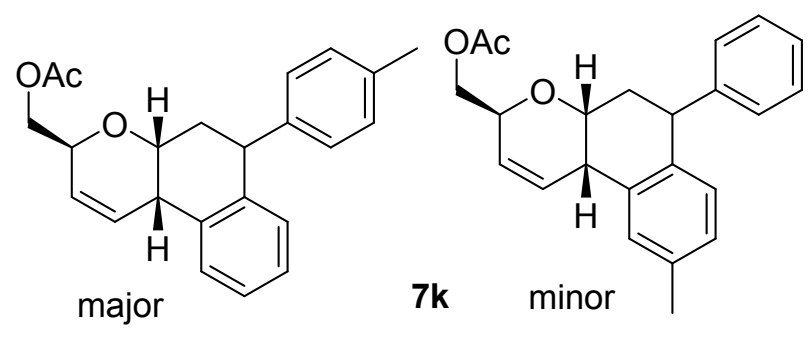


Prepared by general procedure B using tri- $O$-acetyl-D-glucal (100 mg, $0.36 \mathrm{mmol})$, styrene (41.6 $\mu \mathrm{L}, 0.36 \mathrm{mmol})$ and $\mathrm{CF}_{3} \mathrm{SO}_{3} \mathrm{H}(9.5 \mu \mathrm{L}, 0.1 \mathrm{mmol})$ in toluene. Isolated by flash column chromatography (Hexane: EtOAc 49:1) to afford $\mathbf{7 k}$ as a mixture of regioisomers in the ratio of $1.4: 1$ as a colourless oil in $66 \%$ yield, $82 \mathrm{mg}$.

${ }^{1} \mathbf{H}$ NMR (400 MHz, $\left.\mathbf{C D C l}_{3}\right) \delta 7.28$ (dd, $J=7.4,4.7 \mathrm{~Hz}, 2 \mathrm{H}$; minor isomer), 7.27-7.21 (m, 2H), $7.20-7.14(\mathrm{~m}, 2 \mathrm{H}), 7.11$ (s, 2H; minor isomer), $7.08(\mathrm{~d}, J=8.0 \mathrm{~Hz}, 2 \mathrm{H}), 7.04(\mathrm{~d}, J=7.3 \mathrm{~Hz}, 1 \mathrm{H}$; minor isomer), $6.97(\mathrm{~d}, J=8.0 \mathrm{~Hz}, 1 \mathrm{H}), 6.86(\mathrm{t}, J=9.6 \mathrm{~Hz}, 1 \mathrm{H}), 6.73(\mathrm{~d}, J=7.9 \mathrm{~Hz}, 1 \mathrm{H}$; minor isomer). $6.34-6.26(\mathrm{~m}, 1 \mathrm{H}), 5.78-5.62(\mathrm{~m}, 1 \mathrm{H}), 4.38-4.31(\mathrm{~m}, 3 \mathrm{H}), 4.30-4.24(\mathrm{~m}, 1 \mathrm{H}), 4.09$ $(\mathrm{dd}, J=11.5,3.3 \mathrm{~Hz}, 1 \mathrm{H}), 3.55(\mathrm{~d}, J=11.2 \mathrm{~Hz}, 1 \mathrm{H}), 2.43-2.36(\mathrm{~m}, 1 \mathrm{H}), 2.34(\mathrm{~s}, 1 \mathrm{H}), 2.31(\mathrm{~s}$, 3H), 2.09 (s, 3H), $2.04(\mathrm{dd}, J=11.6,9.9 \mathrm{~Hz}, 1 \mathrm{H}) .{ }^{13} \mathbf{C}$ NMR (101 MHz, CDCl $\mathbf{~}_{3} \delta 171.0,146.3$, $138.6,137.6,135.7,131.2,129.8,129.0,128.8,128.7,128.2,126.5,126.1,124.6,69.3,66.0$, 65.1, 41.9, 37.1, 35.3, 21.0. ${ }^{13} \mathbf{C}$ NMR (101 $\left.\mathbf{~ M H z , ~} \mathbf{C D C l}_{3}\right)$ of minor isomer $\delta$ 143.1, 137.7, 136.1, 135.3, 131.1, 129.7, 129.0, 128.8, 128.3, 127.1, 126.2, 125.3, 124.5, 66.0, 65.1, 41.8, 37.1, 21.1. HRMS (ESI+): $\mathrm{m} / \mathrm{z}$ calcd. for $\mathrm{C}_{23} \mathrm{H}_{24} \mathrm{NaO}_{3}(\mathrm{M}+\mathrm{Na})^{+}: 371.1623$; found 371.1668.

\section{$K_{H} / K_{D}$ Calcualation}

\section{With Respect to Product 7aa}<smiles>C=Cc1ccccc1</smiles>

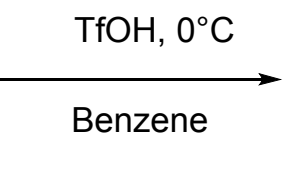

Product 7aa

\begin{tabular}{|c|c|}
\hline Time(min) & Product 7aa \\
\hline 2 & 14.26 \\
\hline 30 & 25.71 \\
\hline 60 & 29.44 \\
\hline 90 & 44.92 \\
\hline 120 & 51.37 \\
\hline
\end{tabular}<smiles>CC(=O)OCC1C=CC=CC=C1</smiles>

7 aa 
With Respect to Product $7 \mathrm{aa}-\mathrm{D}_{6}$
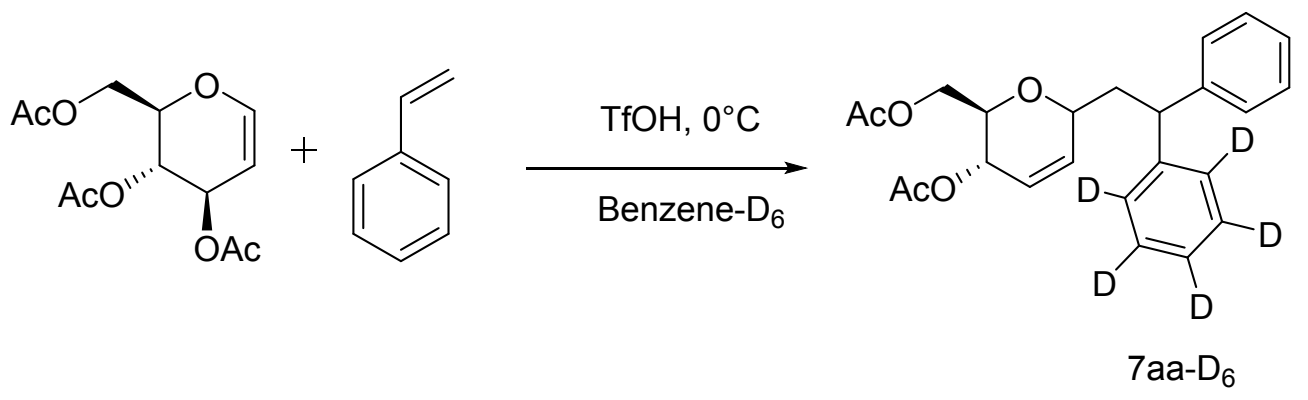

\begin{tabular}{|c|c|}
\hline Time(min) & Product 7aa \\
\hline 2 & 12.92 \\
\hline 30 & 22.32 \\
\hline 60 & 25.16 \\
\hline 90 & 28.99 \\
\hline 120 & 35.68 \\
\hline
\end{tabular}

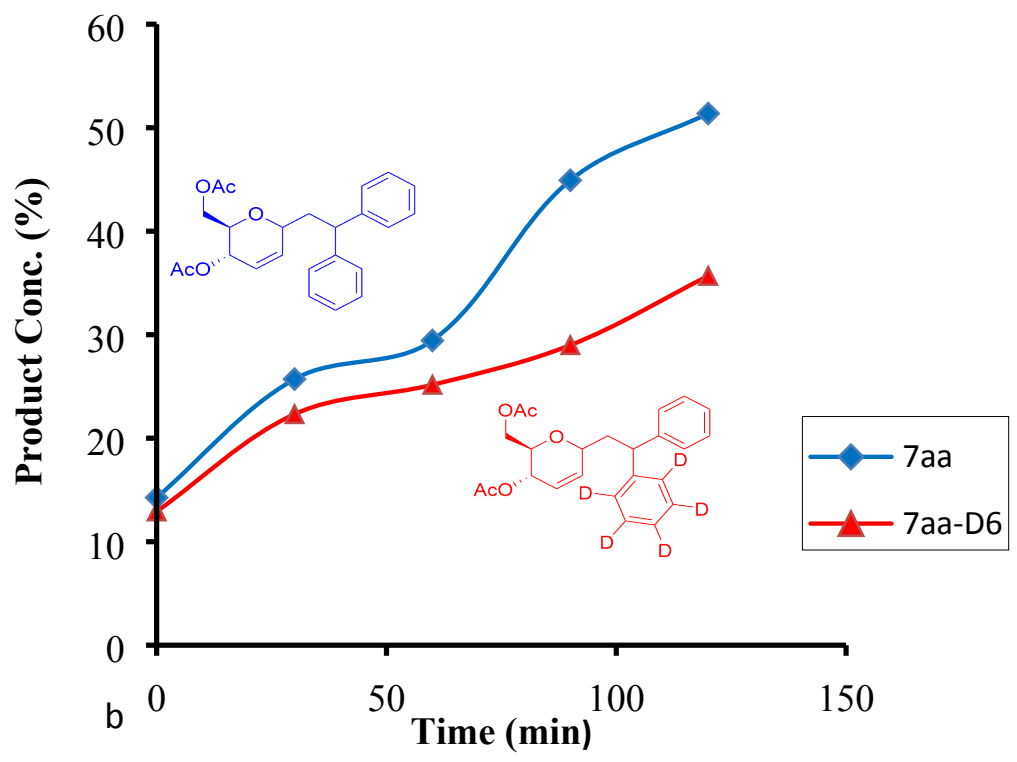

For Standard Product (7aa), intial rate,

$$
\begin{aligned}
& \text { Rate } 1=k_{H}[\mathrm{~A}]^{\mathrm{x}}[\mathrm{B}]^{\mathrm{y}} \\
& 0.124=k_{H}[\mathrm{~A}]^{\mathrm{x}}[\mathrm{B}]^{\mathrm{y}}
\end{aligned}
$$


For Deutrated Product $\left(7 \mathrm{aa}-\mathrm{D}_{6}\right)$, intial rate,

$$
\begin{aligned}
& \text { Rate } 2=k_{D}[\mathrm{~A}]^{\mathrm{x}}[\mathrm{B}]^{\mathrm{y}} \\
& 0.094=k_{D}[\mathrm{~A}]^{\mathrm{x}}[\mathrm{B}]^{\mathrm{y}}
\end{aligned}
$$

Hence from Rate1/Rate2

$$
\begin{gathered}
0.124 / 0.094=\boldsymbol{k}_{H} / \boldsymbol{k}_{\boldsymbol{D}} \\
\boldsymbol{k}_{\boldsymbol{H}} / \boldsymbol{k}_{\boldsymbol{D}}=\mathbf{1 . 3 1}
\end{gathered}
$$




\section{$\underline{\text { Spectral data }}$}

\section{$\underline{1}$ H NMR (400 MHz, $\mathrm{CDCl}_{3}$ ) of compound 2}
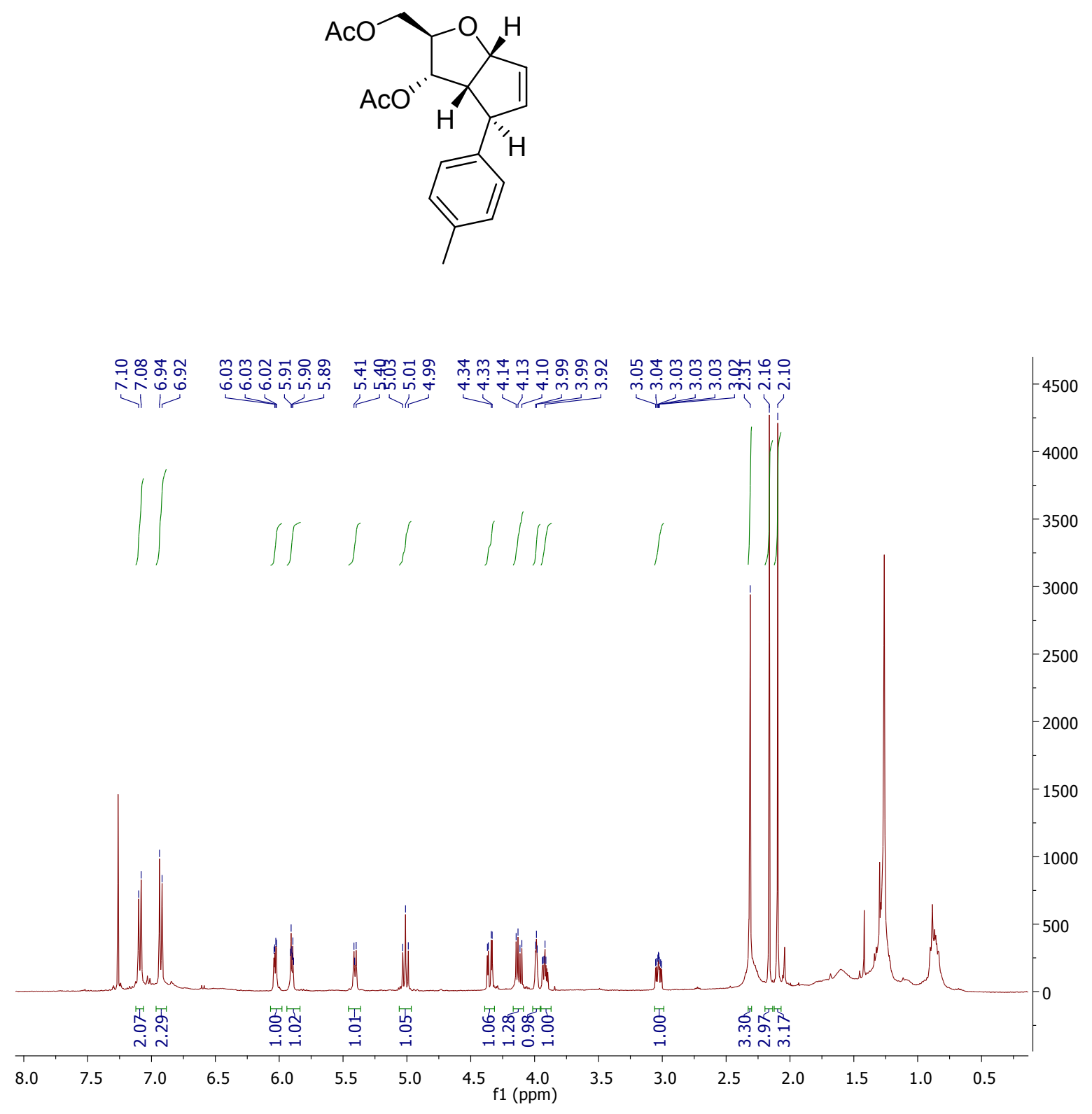
${ }^{13} \mathrm{C}$ NMR (101 MHz, $\left.\mathrm{CDCl} \underline{3}_{3}\right)$ of compound 2<smiles>CC(=O)OC[C@H]1O[C@H]2C=C[C@H](c3ccc(C)cc3)[C@H]2[C@H]1OC(C)=O</smiles>

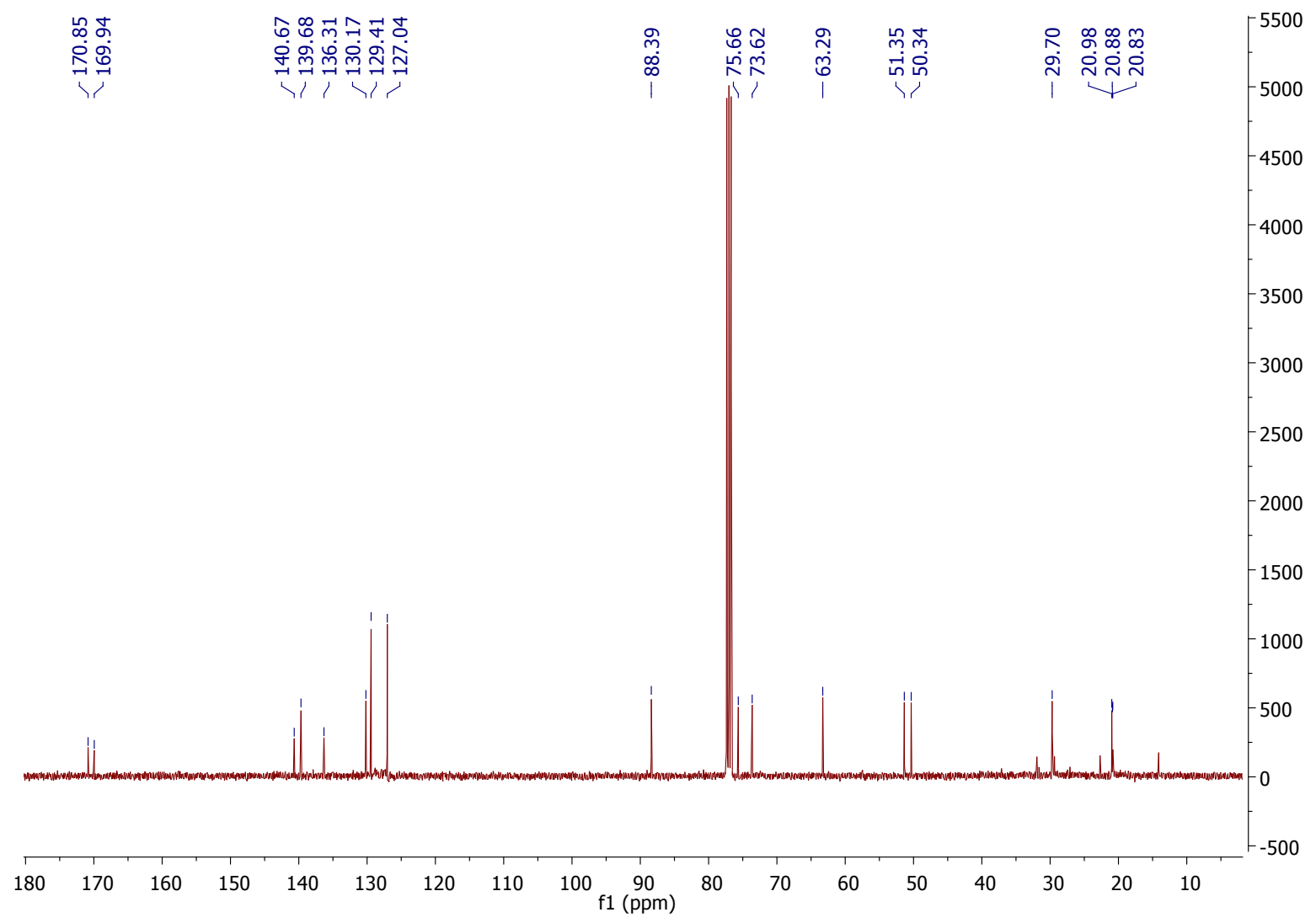


$\underline{\left.{ }^{1} \mathrm{H} \text { NMR (400 MHz, } \mathrm{CDCl}_{3}\right) \text { of compound 2a }}$
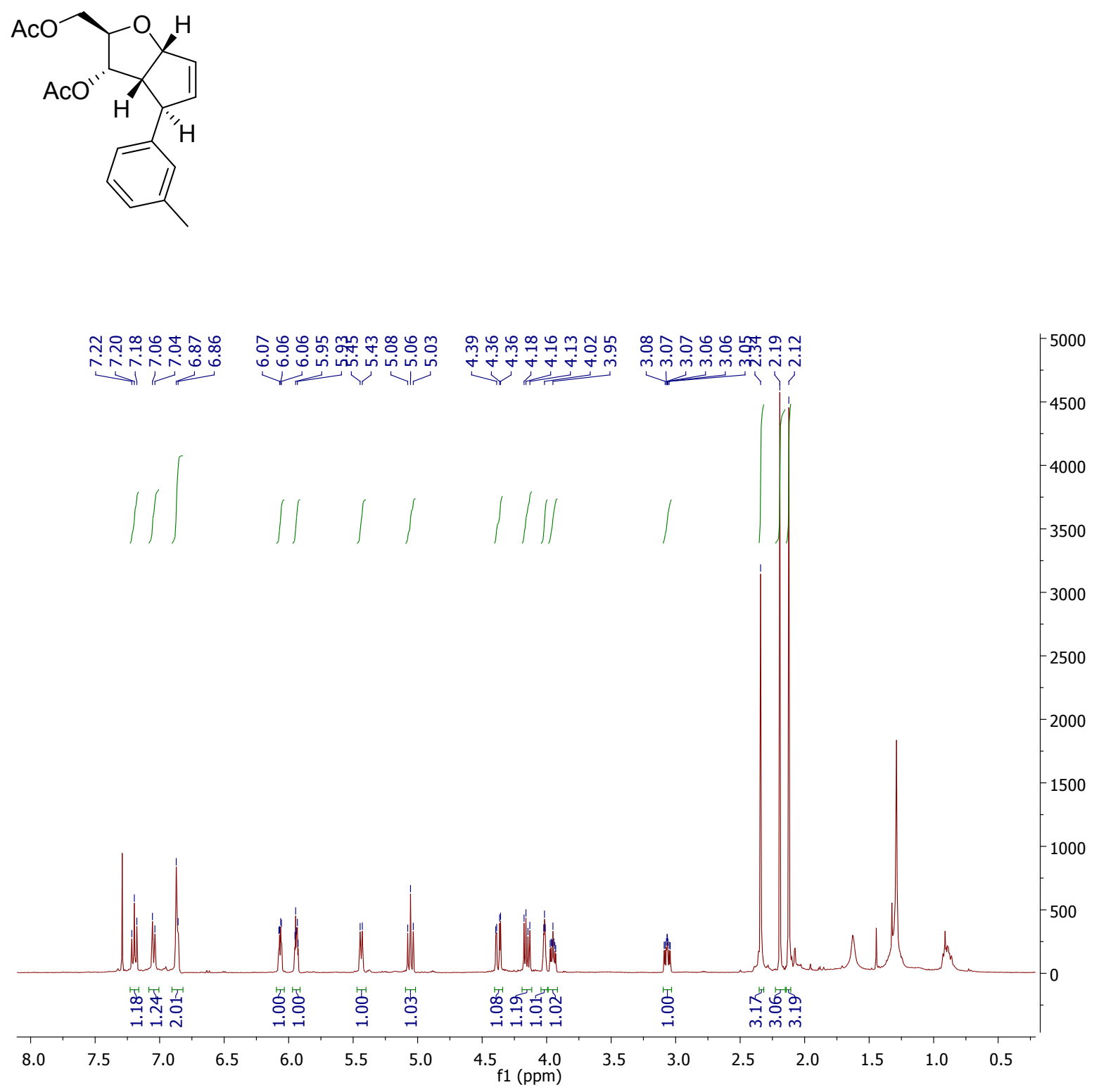


\section{$\underline{{ }^{13} \mathrm{C} \text { NMR (101 MHz, } \mathrm{CDCl}_{3} \text { ) of compound 2a }}$}

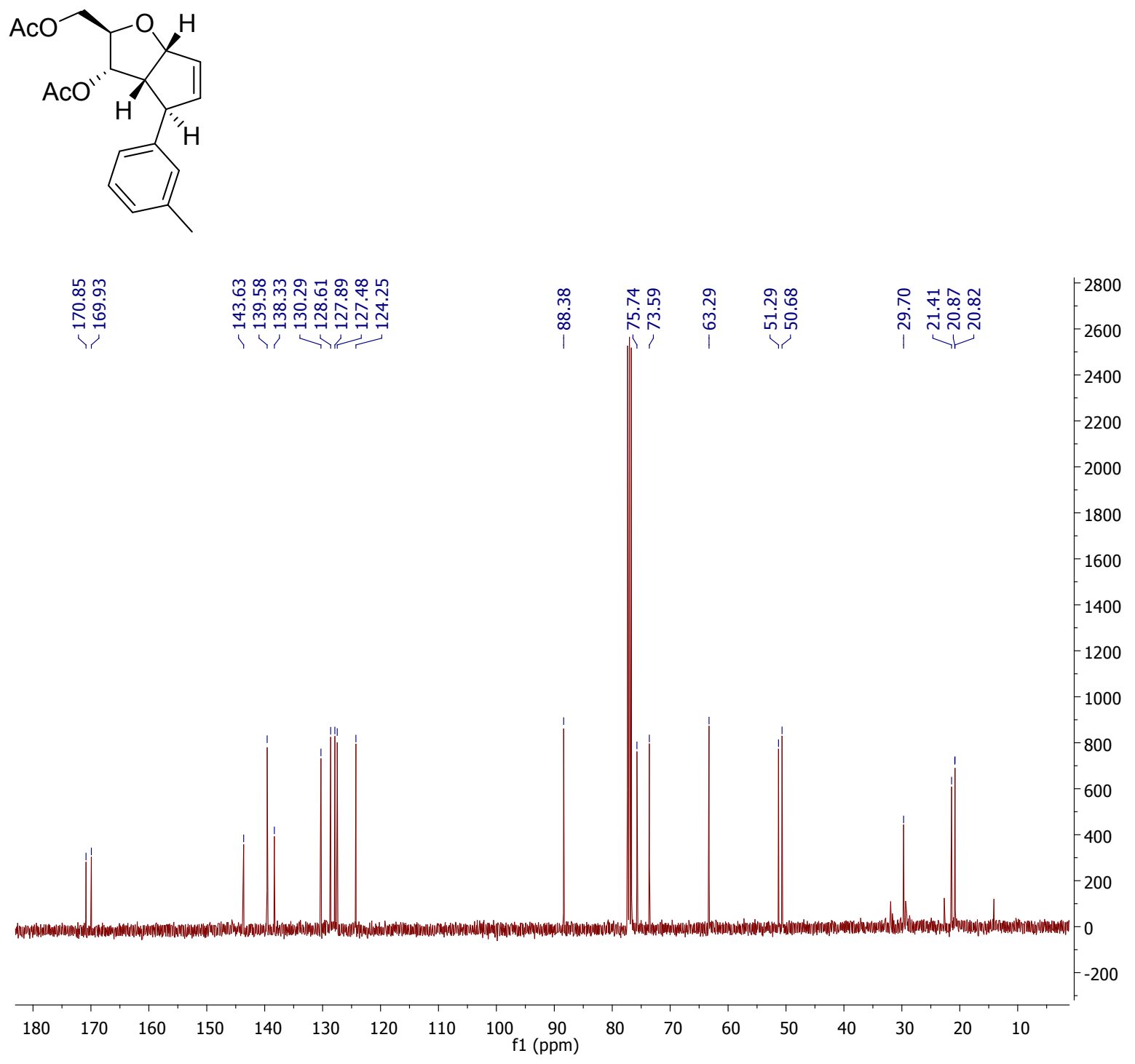




\section{$\underline{1 \mathrm{H} \text { NMR (400 MHz, } \mathrm{CDCl}_{3} \text { ) of compound } 2 \mathrm{~b}}$}

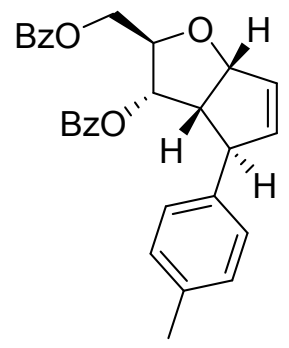

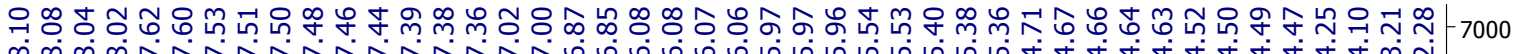

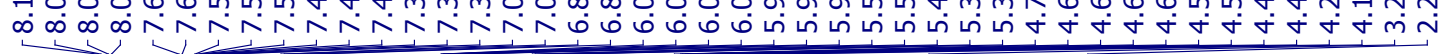

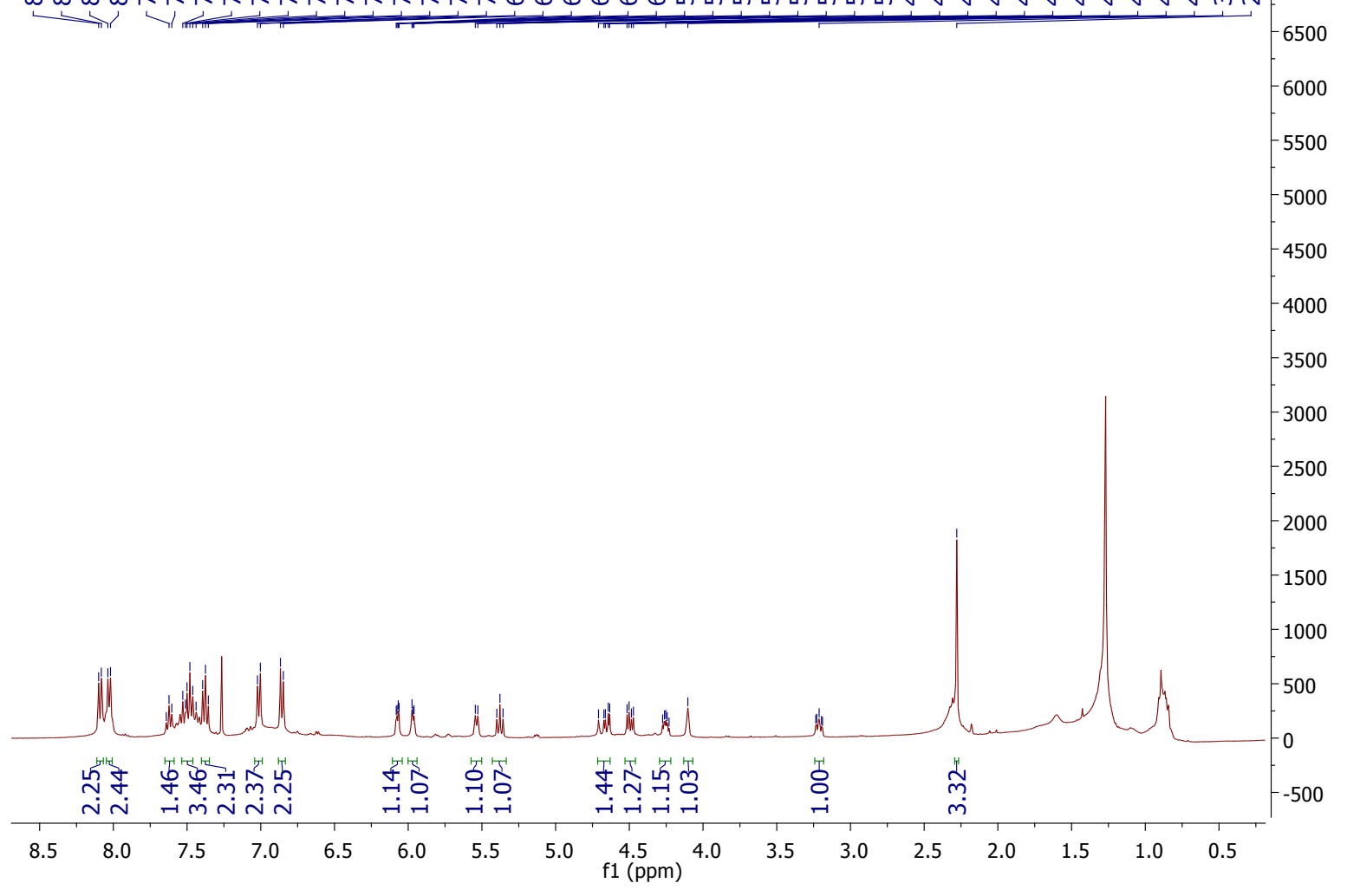


${ }^{13} \mathrm{C}$ NMR (101 MHz, $\mathrm{CDCl}_{3}$ ) of compound $2 \mathrm{~b}$

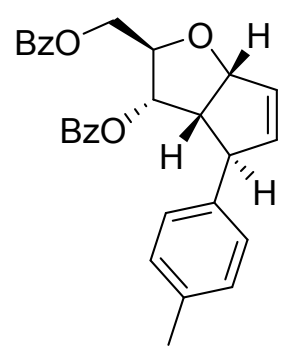

mo

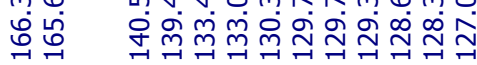

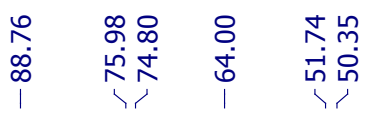

in

$-6500$

6000

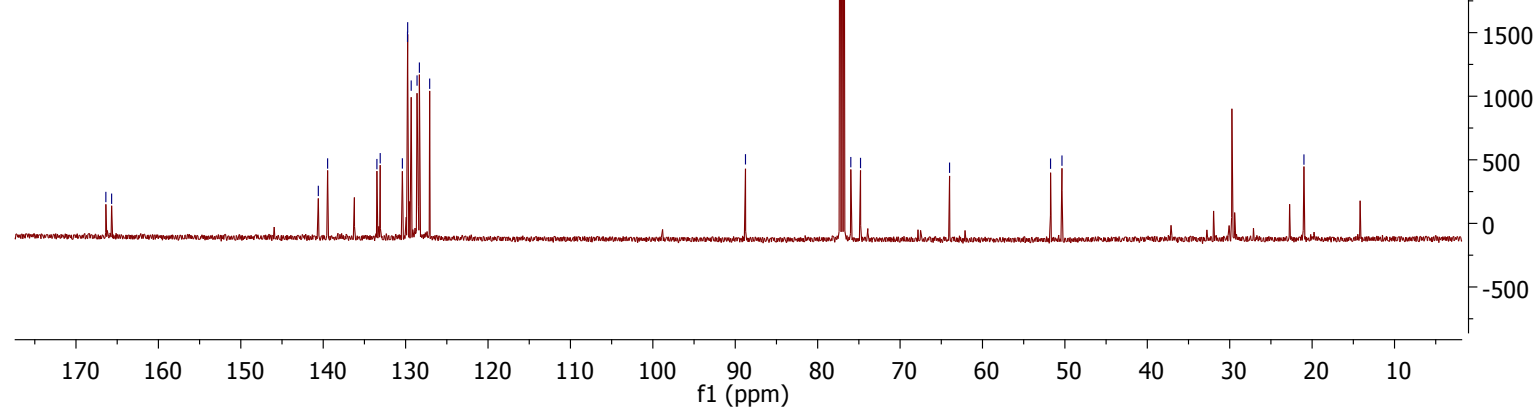




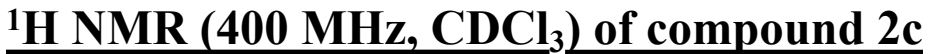
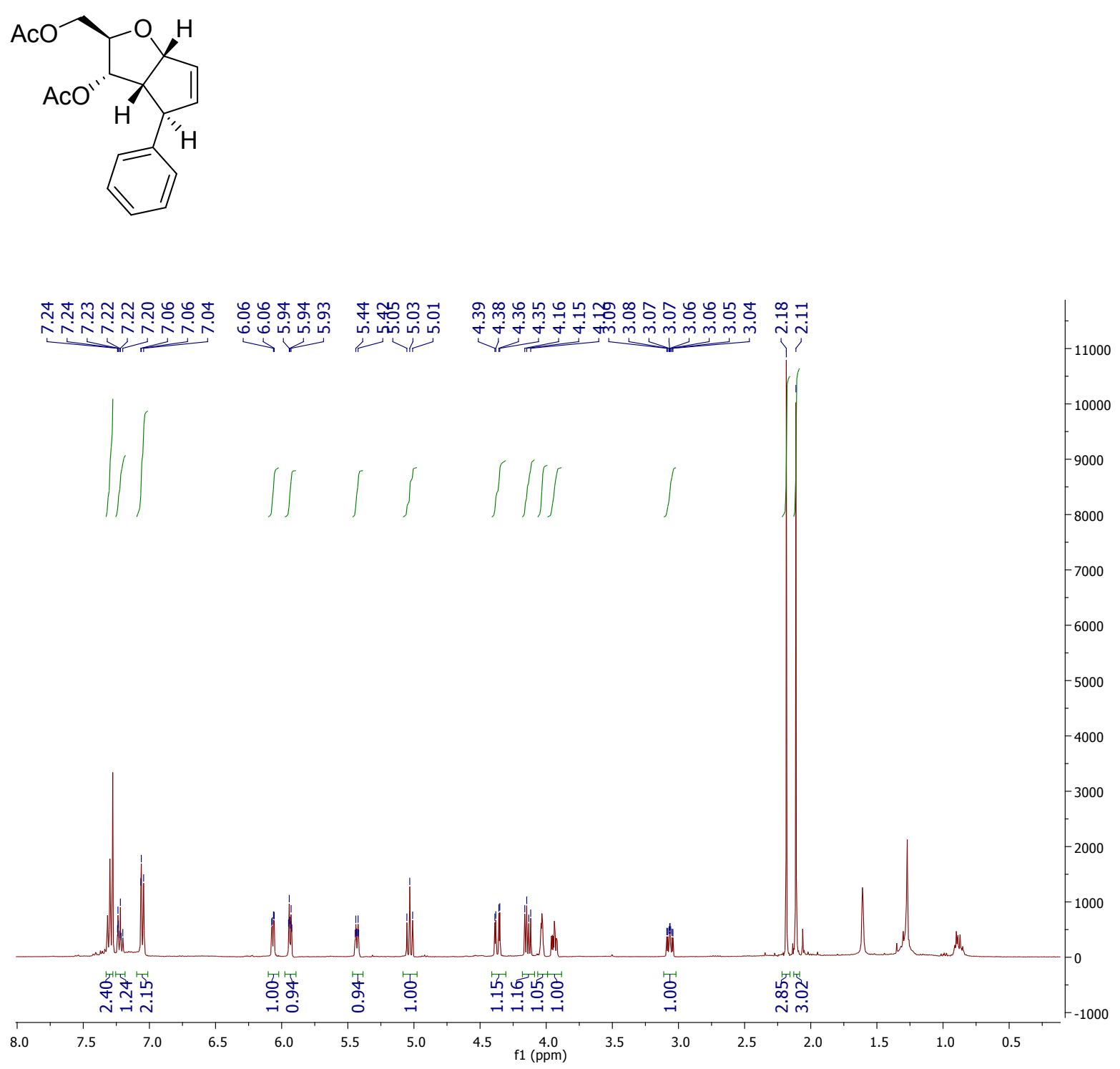
${ }^{13} \mathrm{C}$ NMR (101 MHz, $\left.\mathrm{CDCl}_{3}\right)$ of compound $2 \mathrm{c}$
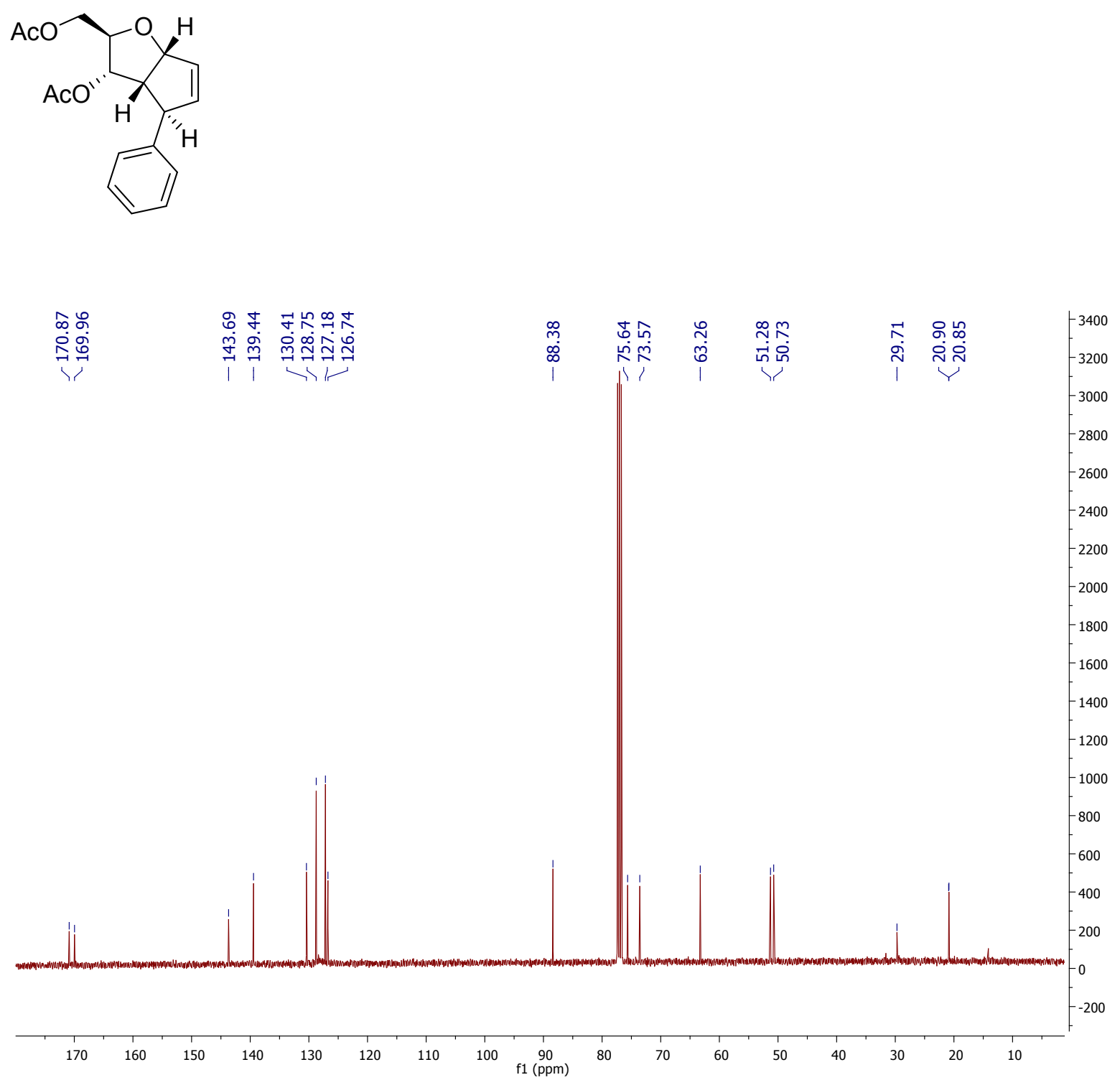


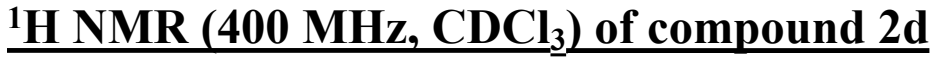
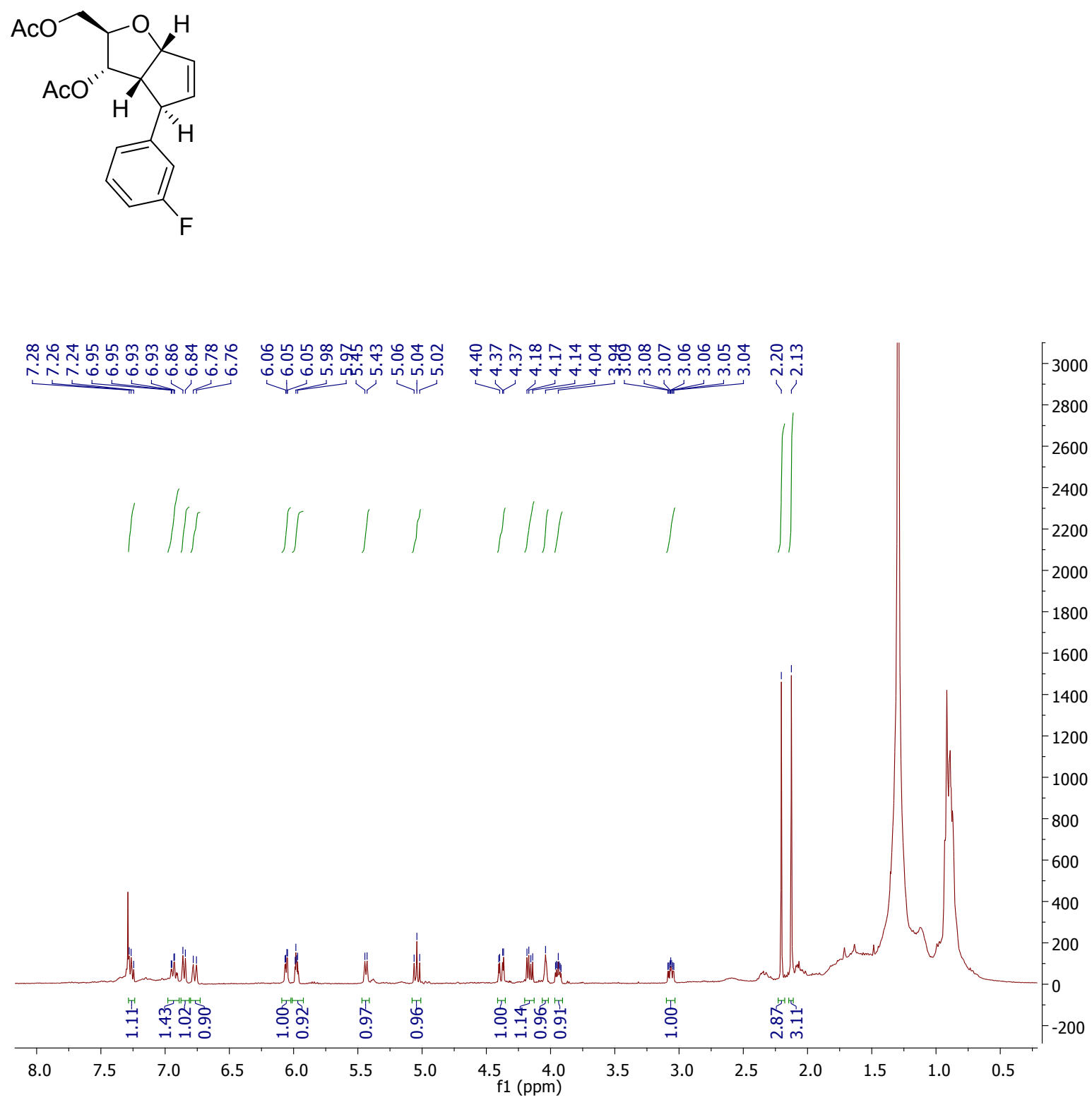


\section{${ }^{13} \mathrm{C}$ NMR (101 MHz, $\mathrm{CDCl}_{3}$ ) of compound 2d}

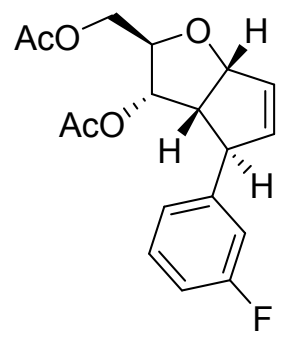

ळक्ष

윙

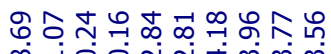

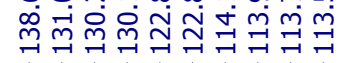

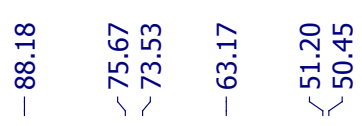

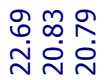

$-3800$

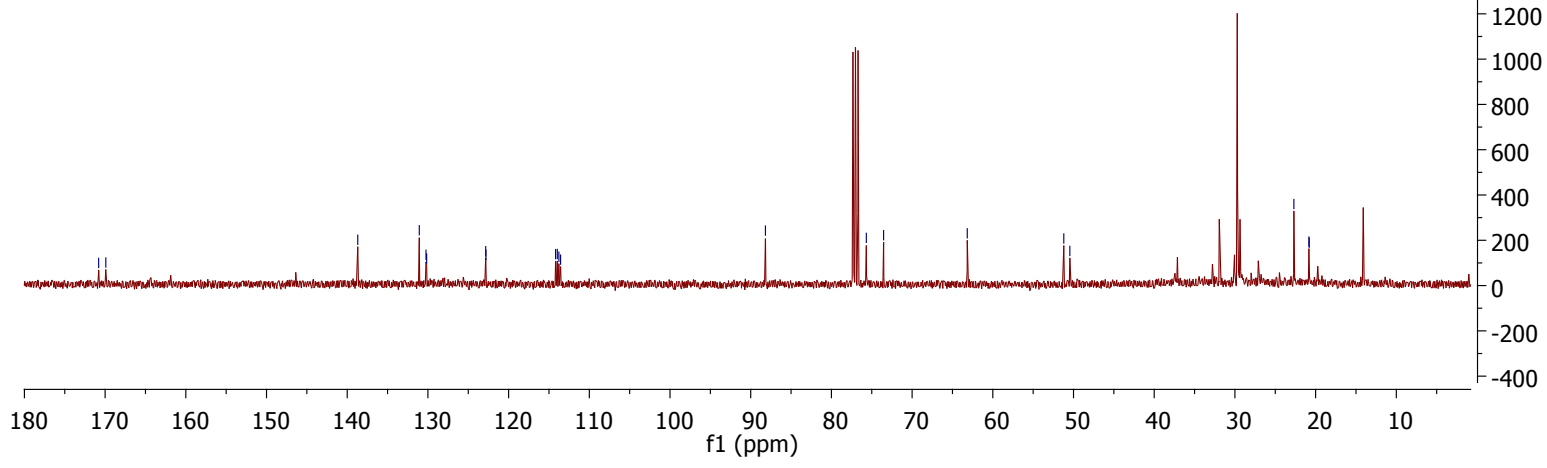




\section{$\underline{\left.{ }^{1} \mathrm{H} \text { NMR (400 MHz, } \mathrm{CDCl}_{3}\right) \text { of compound } 2 \mathrm{e}}$}

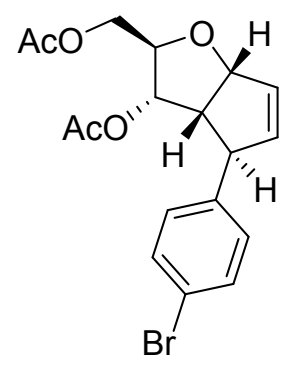

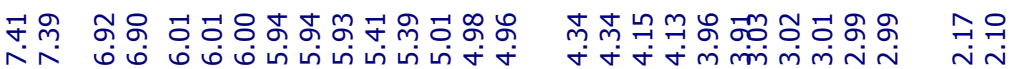

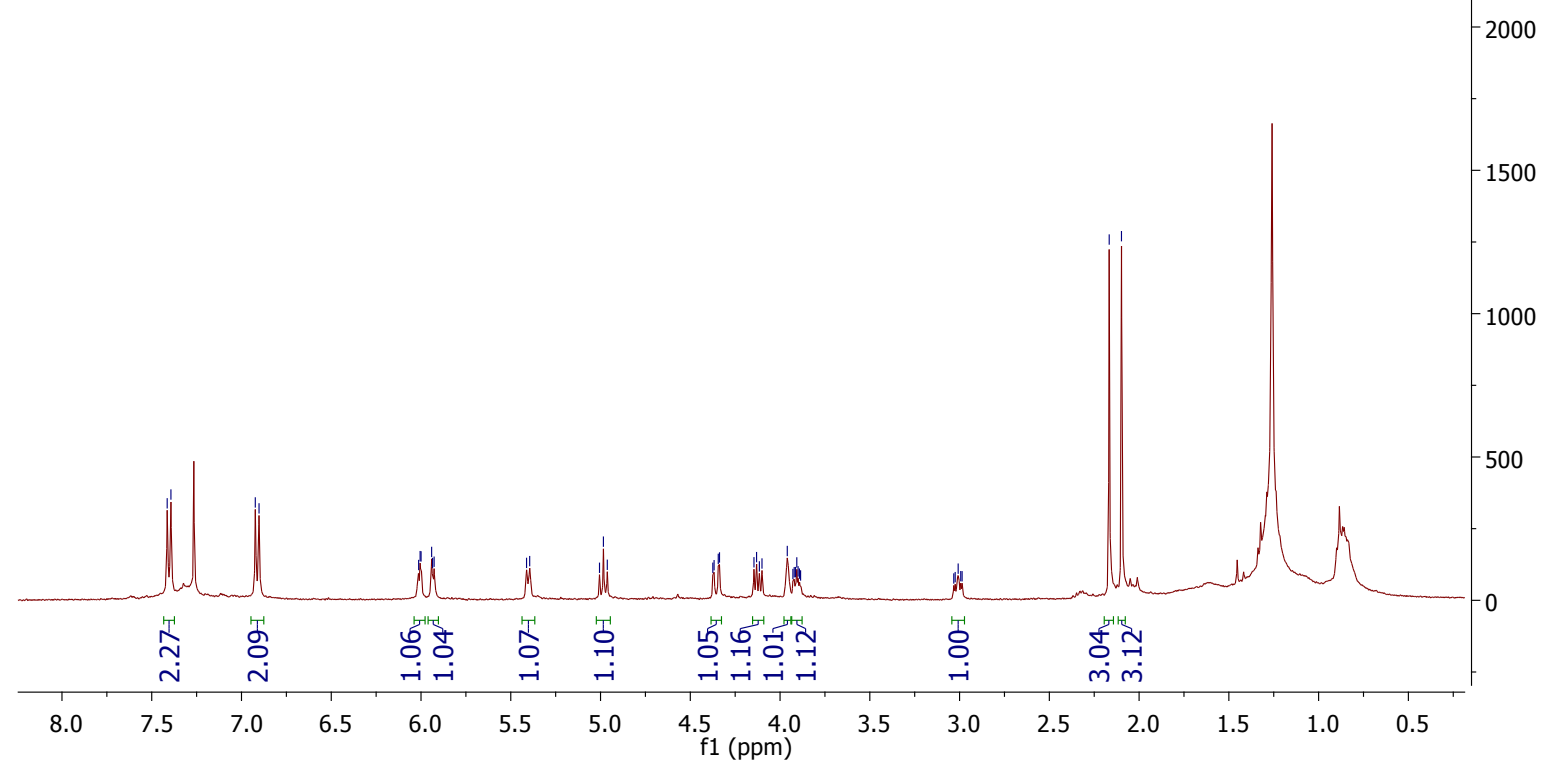




\section{${ }^{13} \mathrm{C}$ NMR (101 MHz, $\mathrm{CDCl}_{3}$ ) of compound 2e}
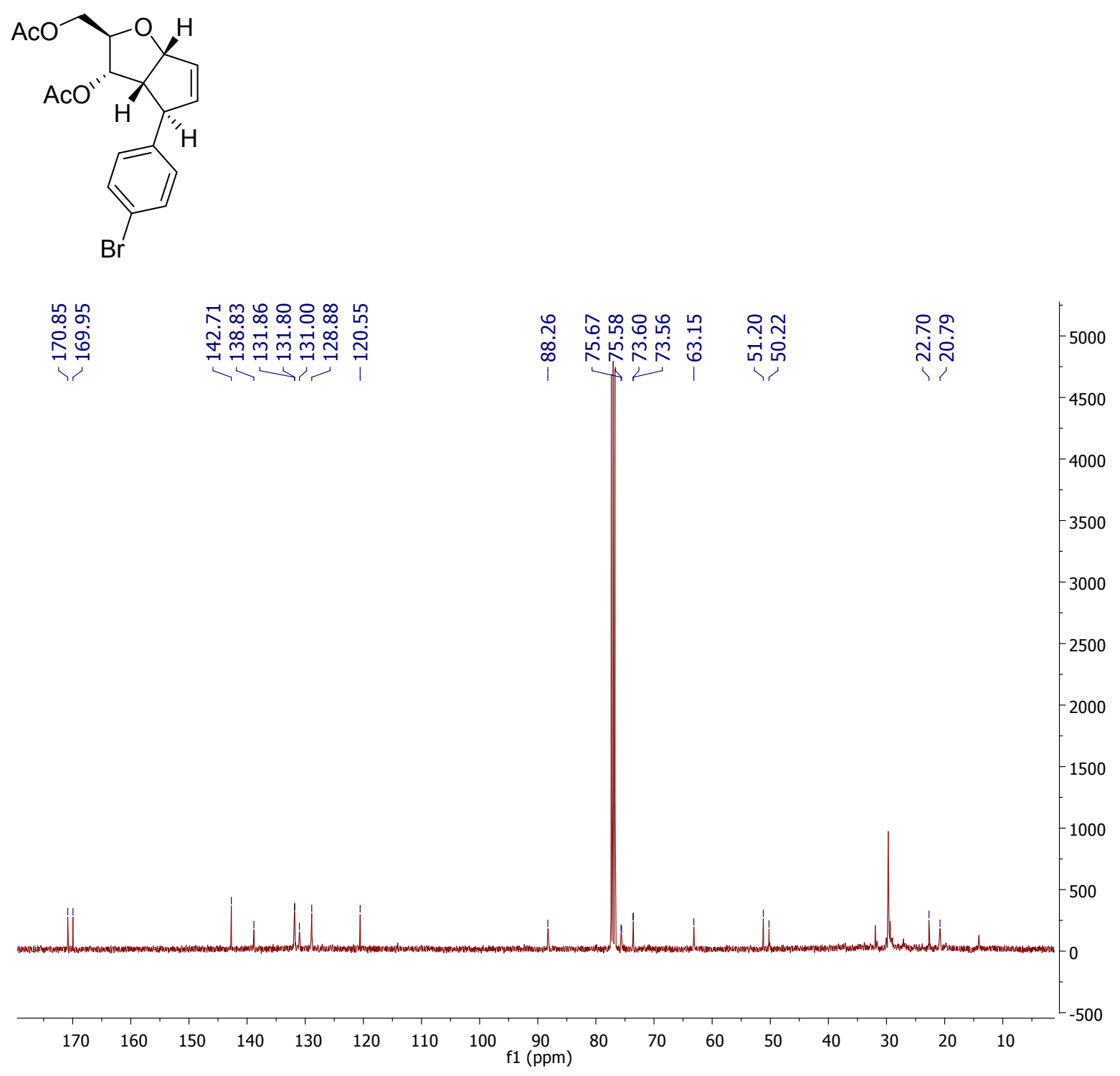


\section{$\underline{{ }^{1} \mathrm{H} \text { NMR }\left(400 \mathrm{MHz}, \mathrm{CDCl}_{3}\right) \text { of compound } 2 \mathrm{f}}$}<smiles>CC(=O)OC[C@H]1O[C@@H]2C=C[C@H](c3ccc(Cl)cc3)[C@H]2[C@H]1[14CH3]</smiles>

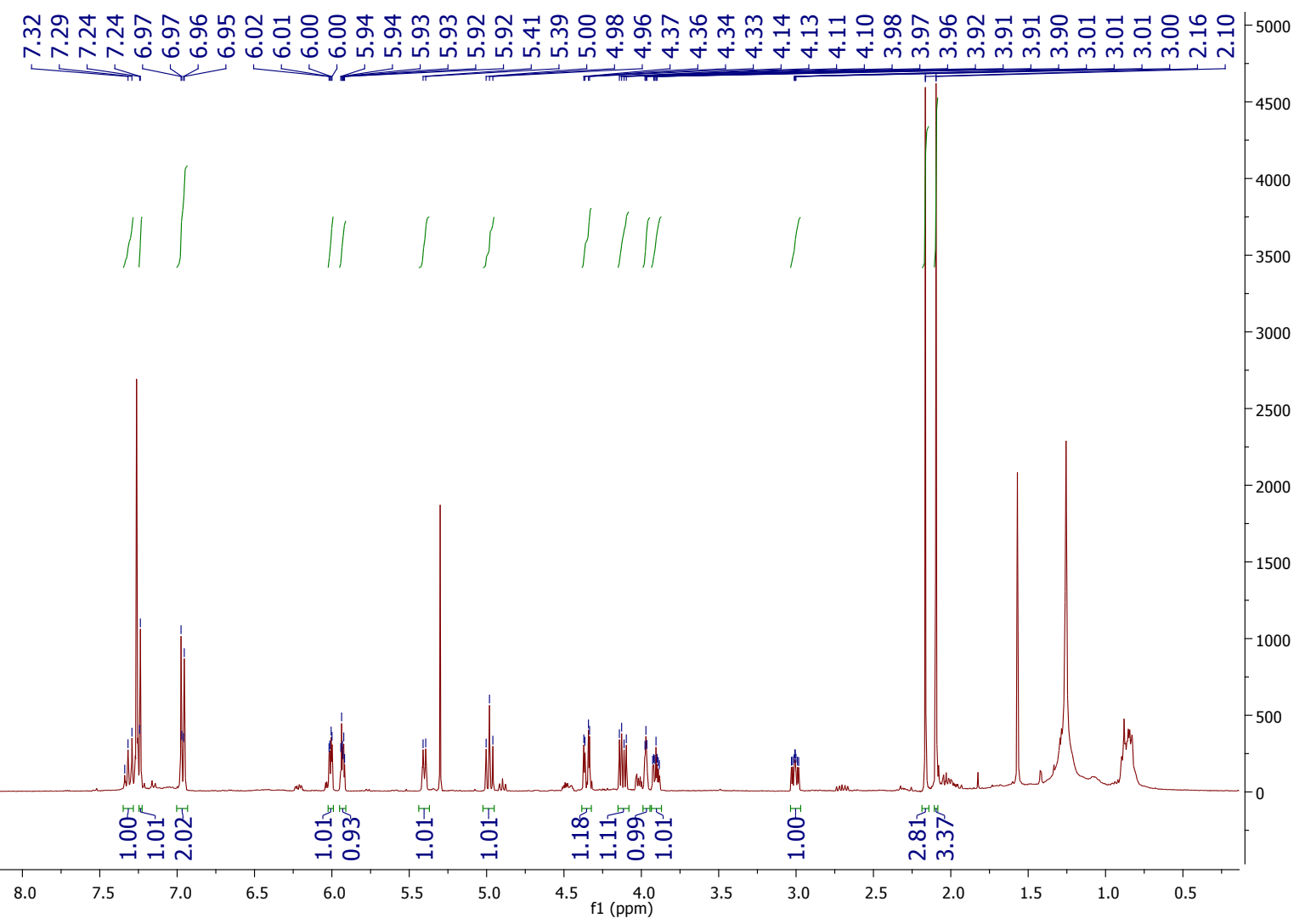




\section{${ }^{13} \mathrm{C}$ NMR (101 MHz, $\mathrm{CDCl}_{3}$ ) of compound $2 \mathrm{f}$}
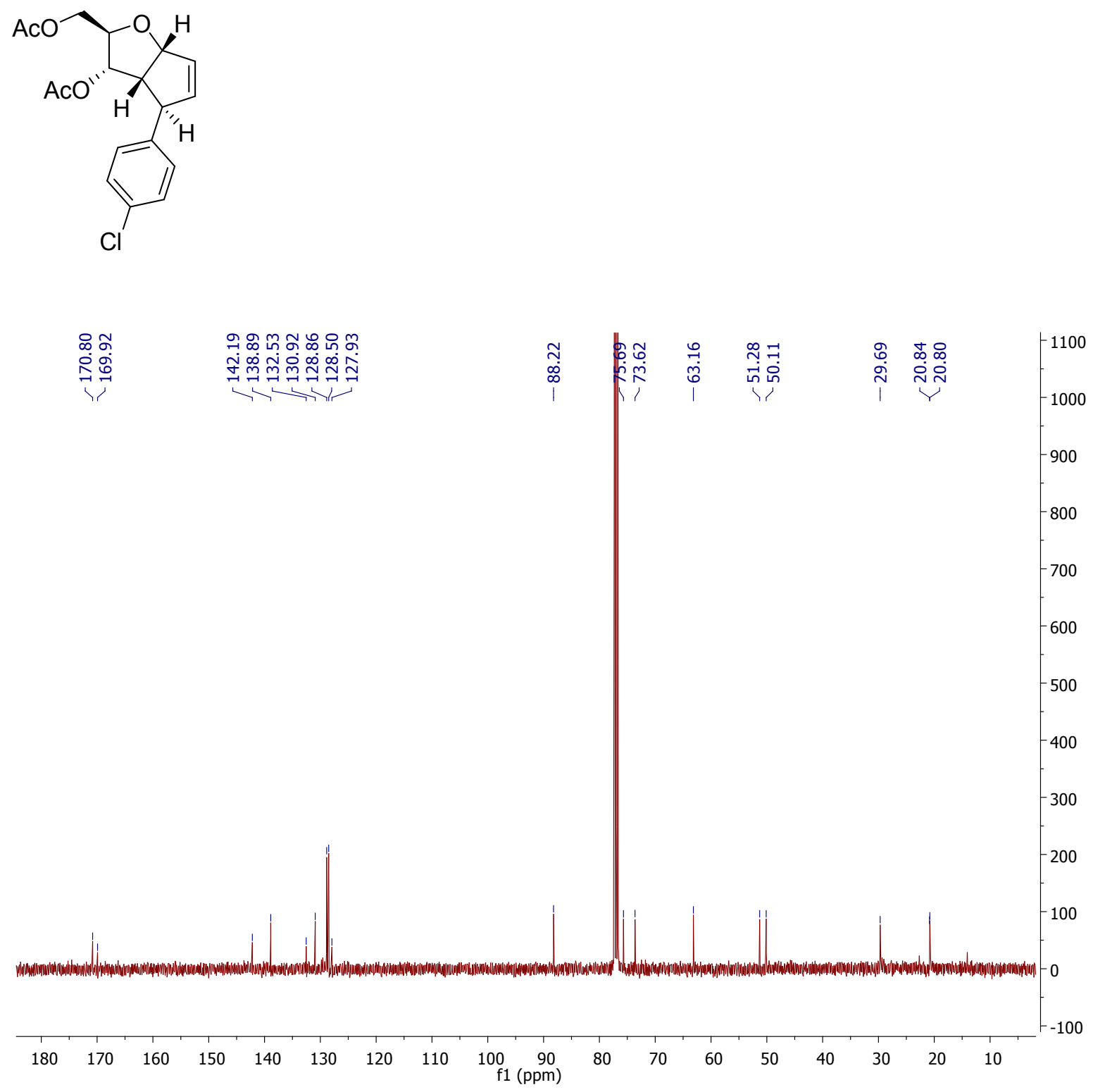


\section{$\underline{{ }^{1} \mathrm{H}}$ NMR (400 MHz, $\mathrm{CDCl}_{3}$ ) of compound $2 \mathrm{~g}$}
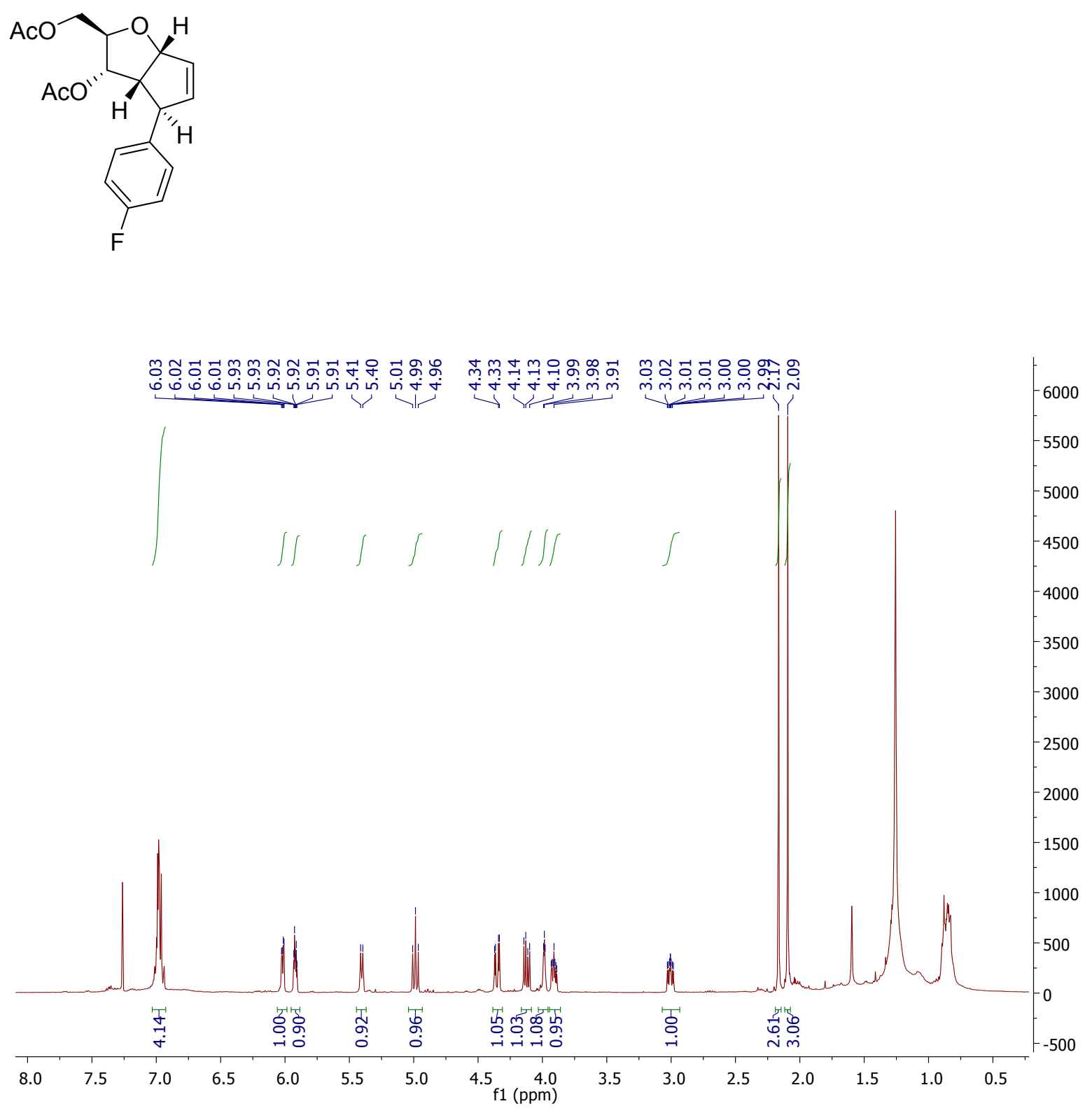


\section{${ }^{13} \mathrm{C}$ NMR (101 MHz, $\mathrm{CDCl}_{3}$ ) of compound $2 \mathrm{~g}$}
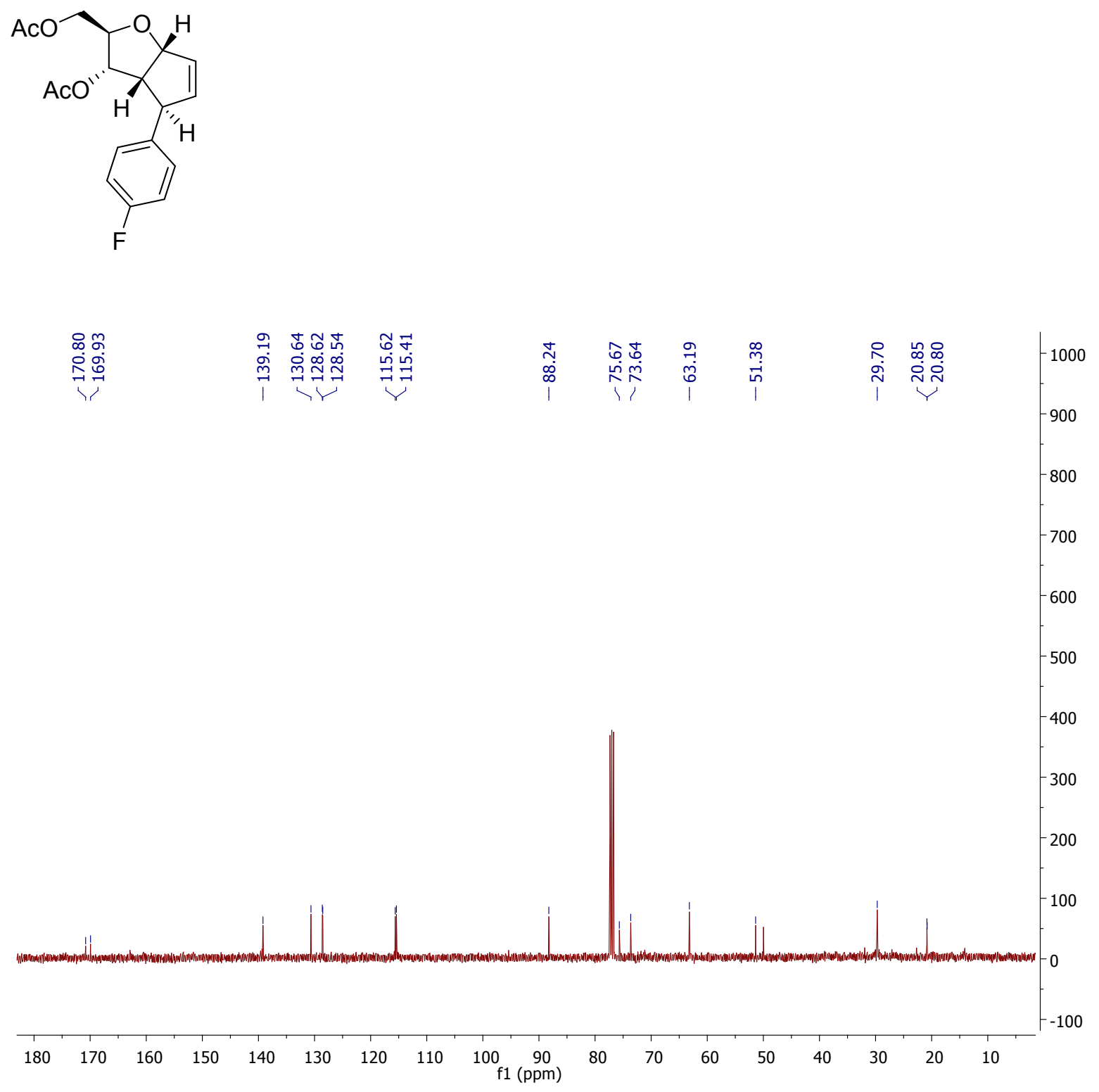


\section{$\underline{{ }^{1} \mathrm{H} \text { NMR (400 MHz, } \mathrm{CDCl}_{3} \text { ) of compound } 2 \mathrm{~h}}$}<smiles>CC(=O)OC[C@H]1O[C@@H]2C=C[C@H](c3ccccc3Cl)[C@H]2[C@H]1OC(C)=O</smiles>

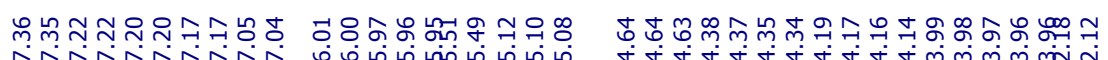

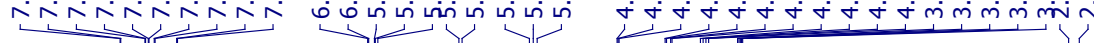
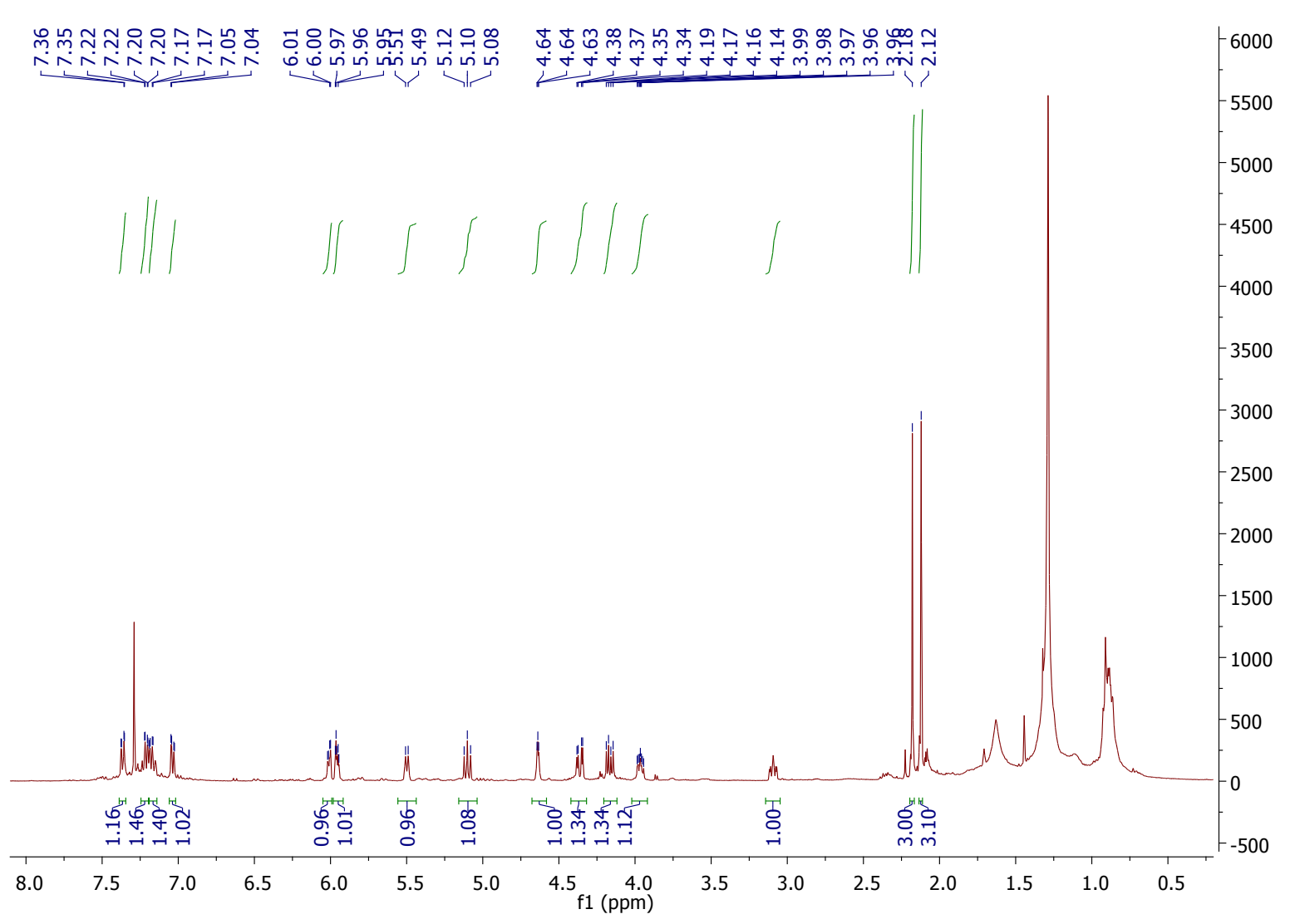
$\underline{{ }^{13} \mathrm{C} \text { NMR (101 MHz, } \mathrm{CDCl}_{3} \text { ) of compound } 2 \mathrm{~h}}$

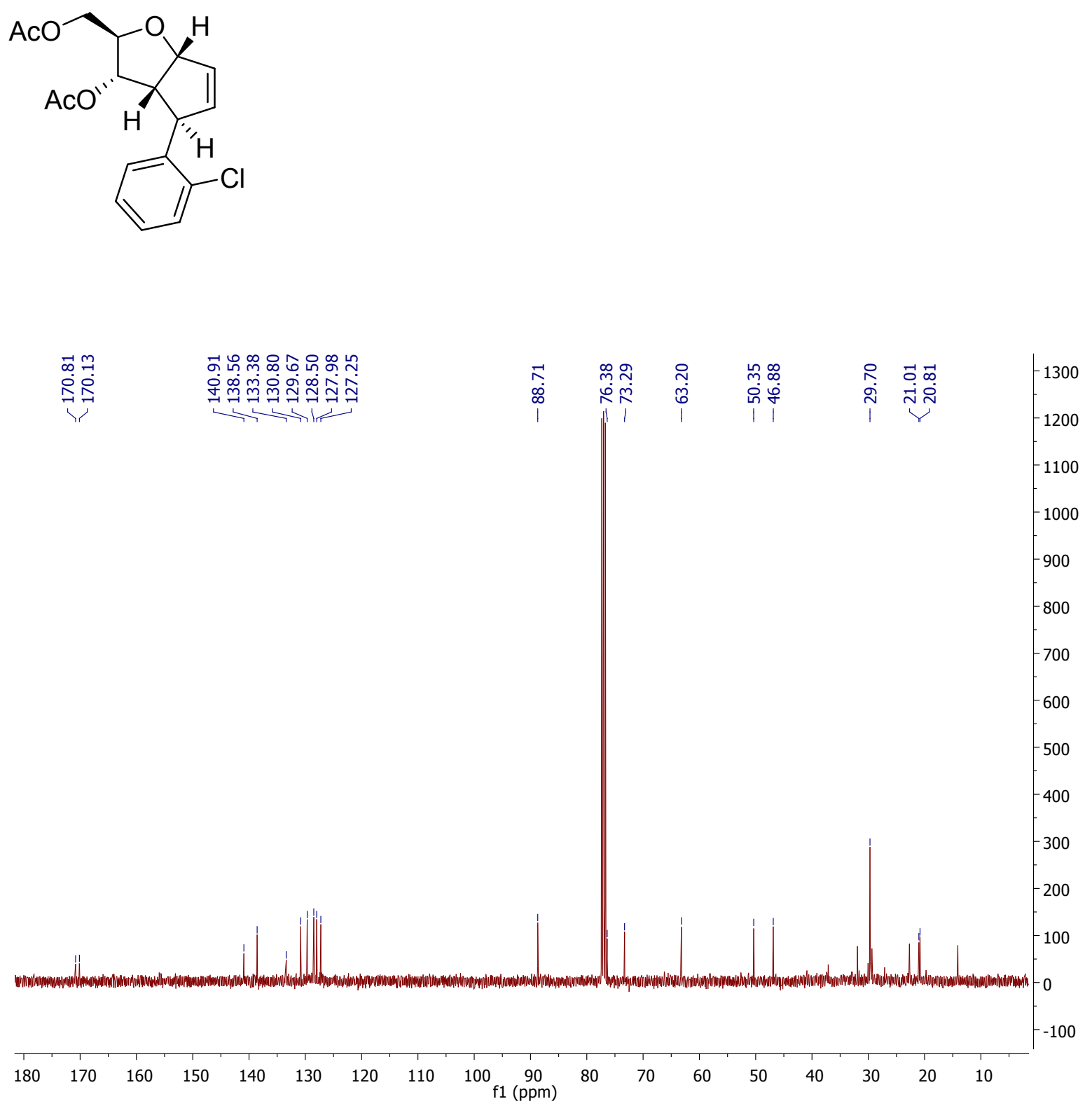




\section{$\underline{\left.{ }^{1} \mathrm{H} \text { NMR (400 MHz, } \mathrm{CDCl}_{3}\right) \text { of compound } 2 \mathrm{i}}$}
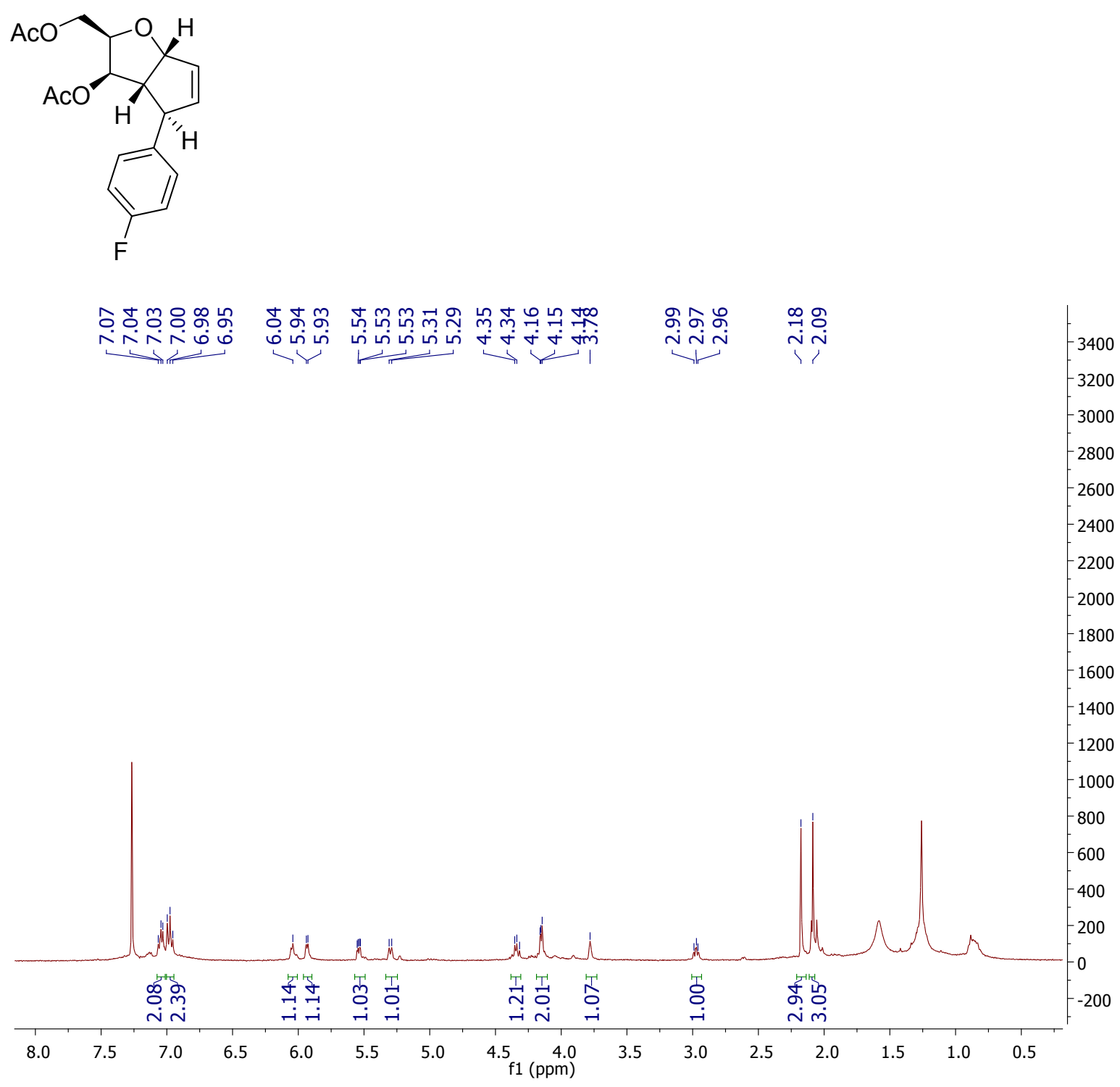


\section{${ }^{13} \mathrm{C}$ NMR (101 MHz, $\mathrm{CDCl}_{3}$ ) of compound $2 \mathrm{i}$}

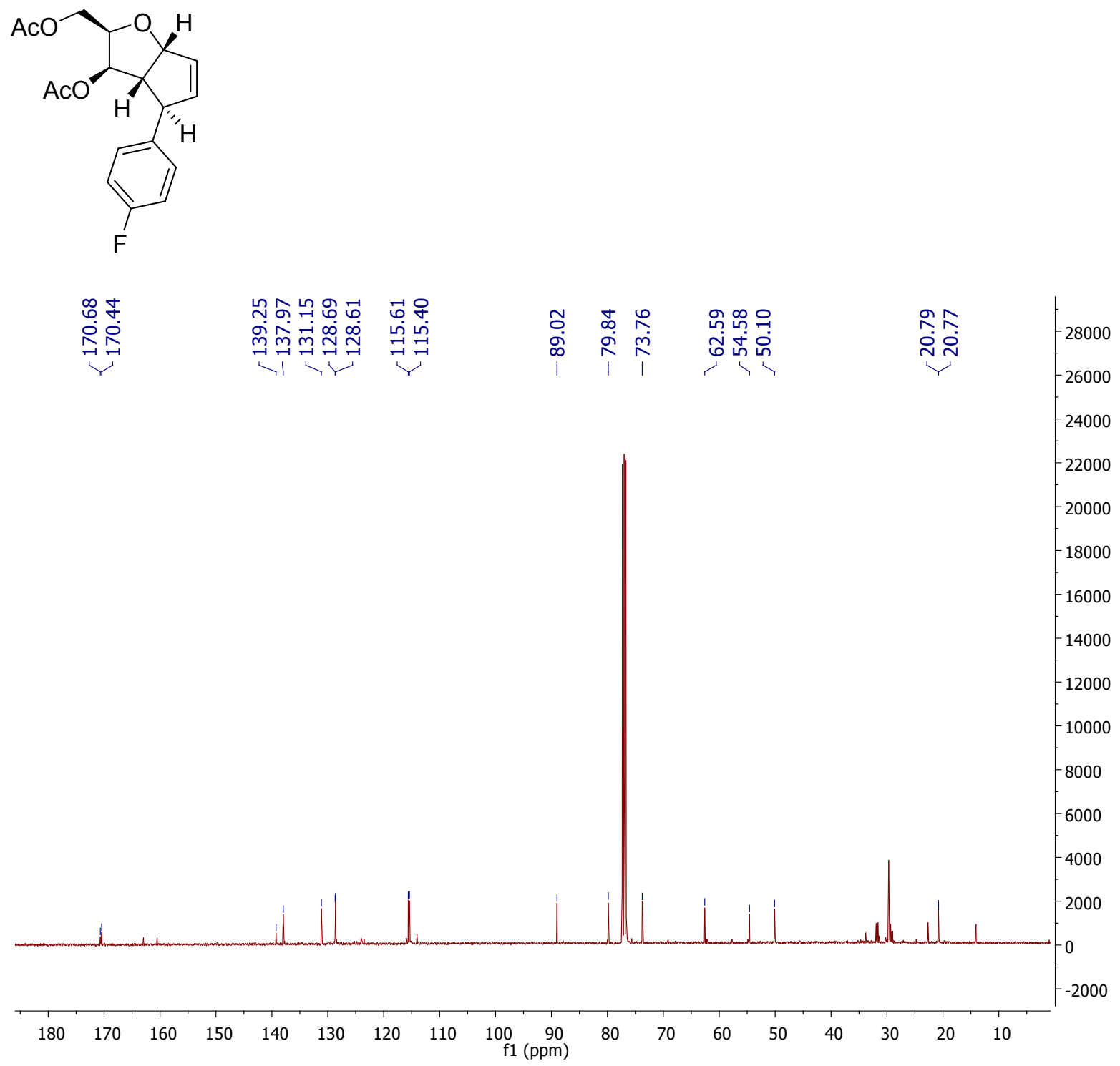




\section{$\underline{{ }^{1} \mathrm{H} \text { NMR }(400 \mathrm{MHz}, \mathrm{CDCl}} \underline{3}$ ) of compound $2 \mathrm{j}$}

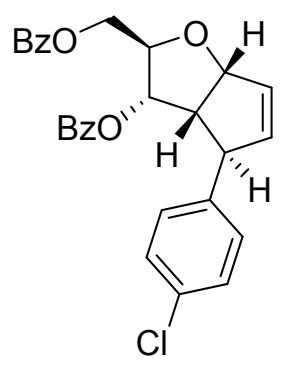

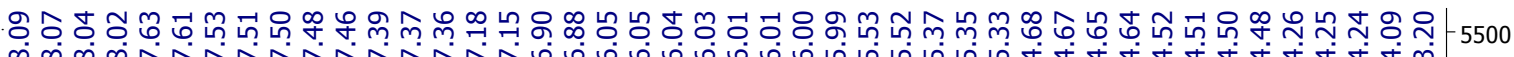

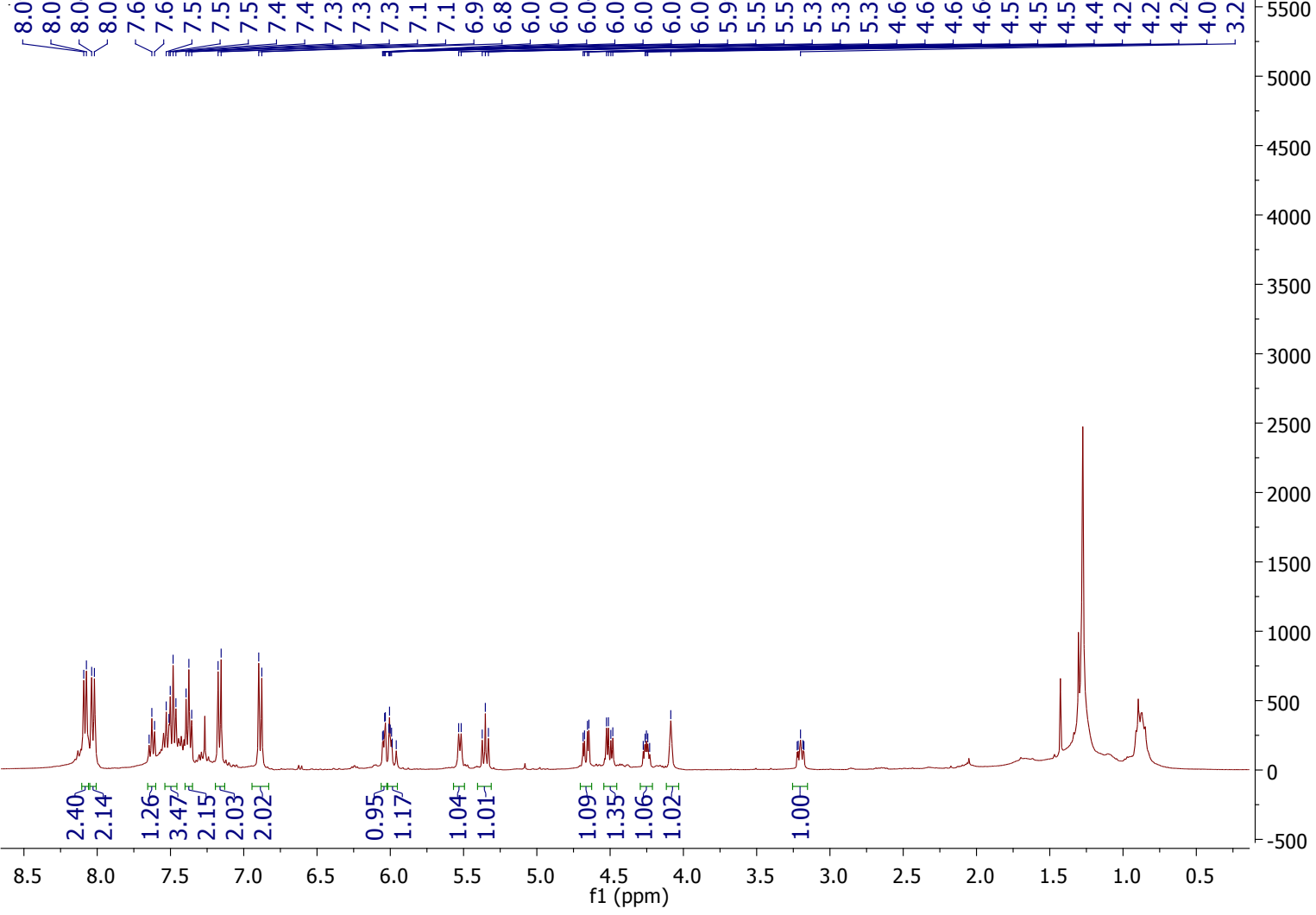




\section{${ }^{13} \mathrm{C}$ NMR (101 MHz, $\mathrm{CDCl}_{3}$ ) of compound $2 \mathrm{i}$}

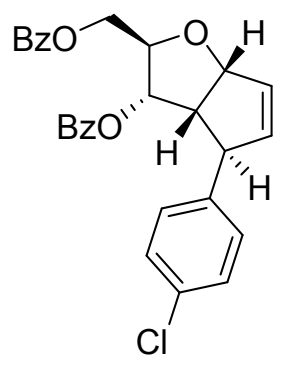

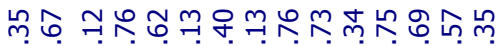

完它 孚

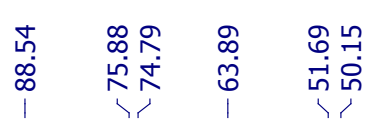

6000

5500

5000

4500

4000

3500

3000

2500

2000

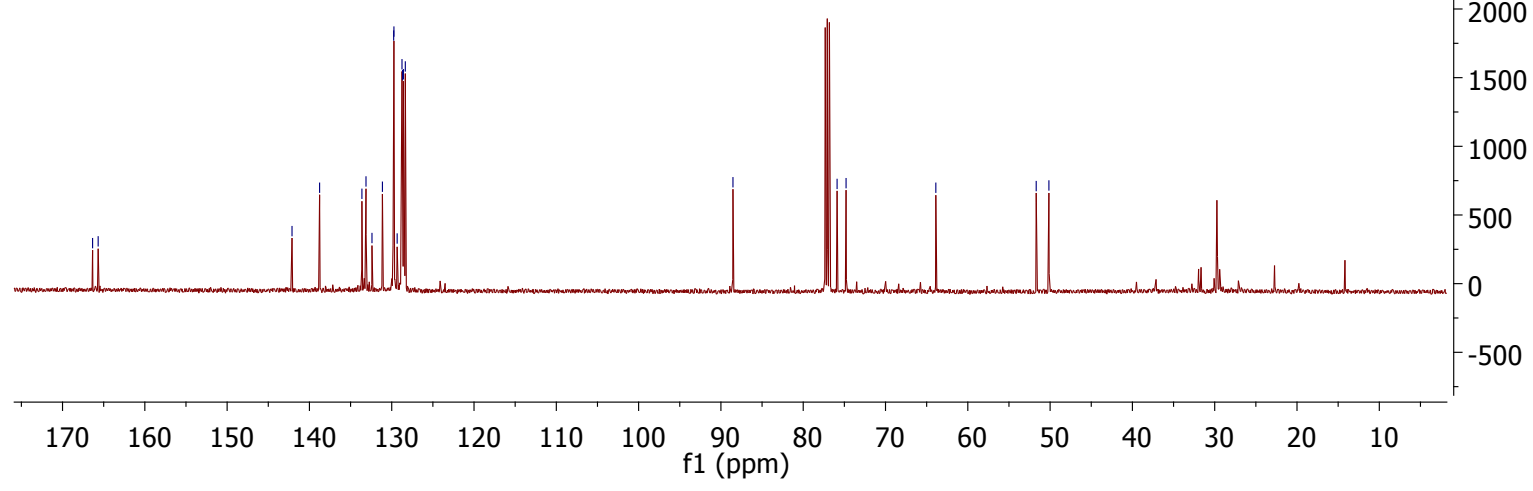




\section{$\underline{1 \mathrm{H} \text { NMR (400 MHz, } \mathrm{CDCl}_{3} \text { ) of compound } 2 \mathrm{k}}$}

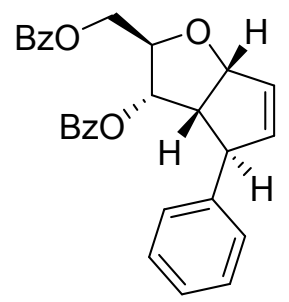

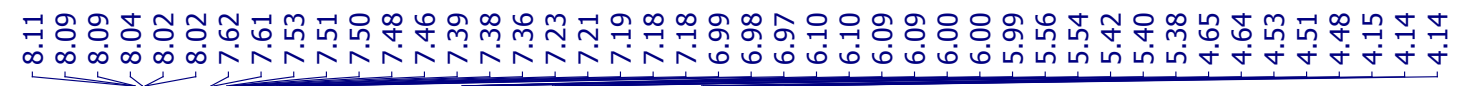

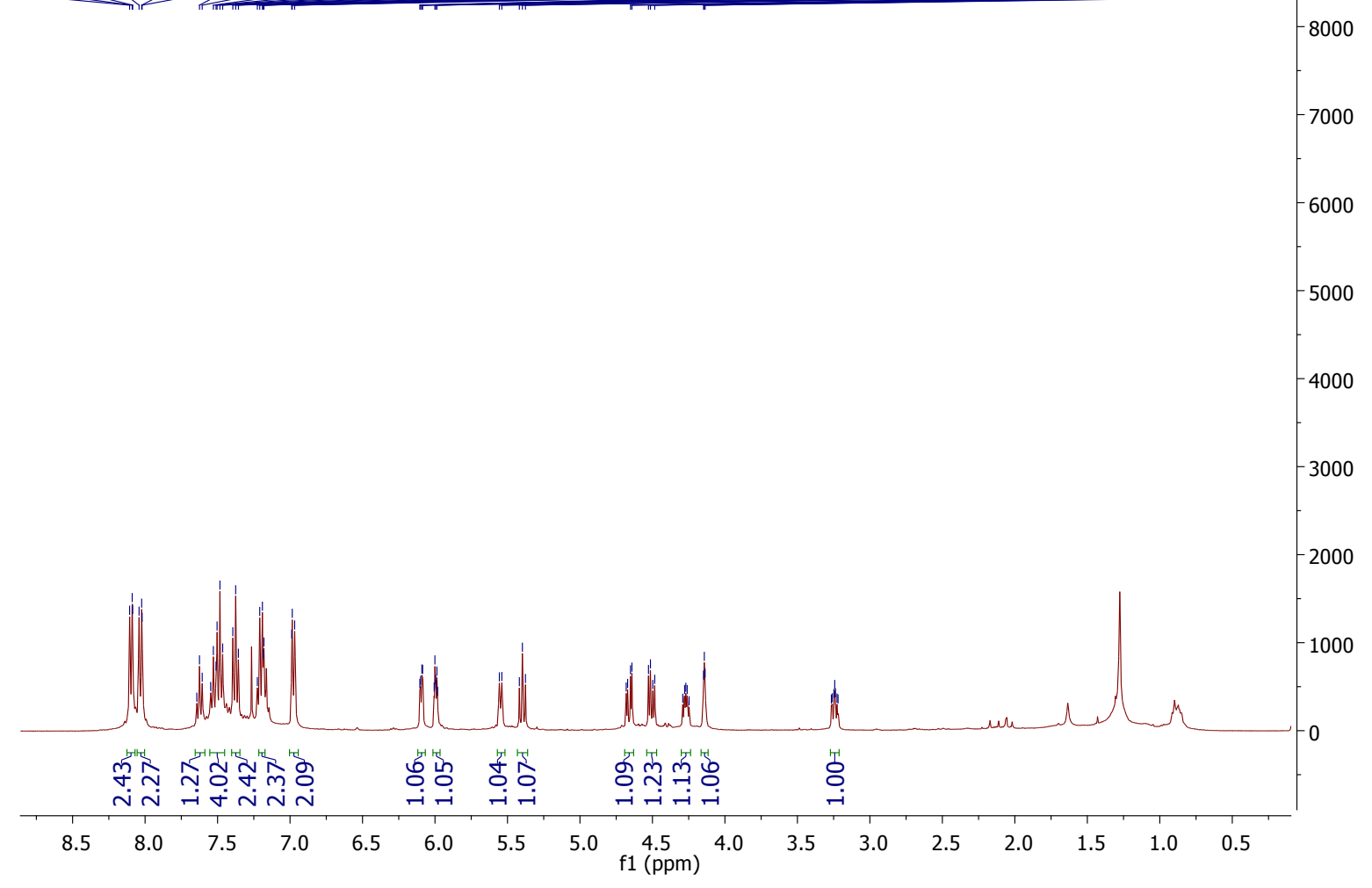




\section{$\underline{\left.{ }^{13} \mathrm{C} \text { NMR (101 MHz, } \mathrm{CDCl}_{3}\right) \text { of compound } 2 \mathrm{k}}$}

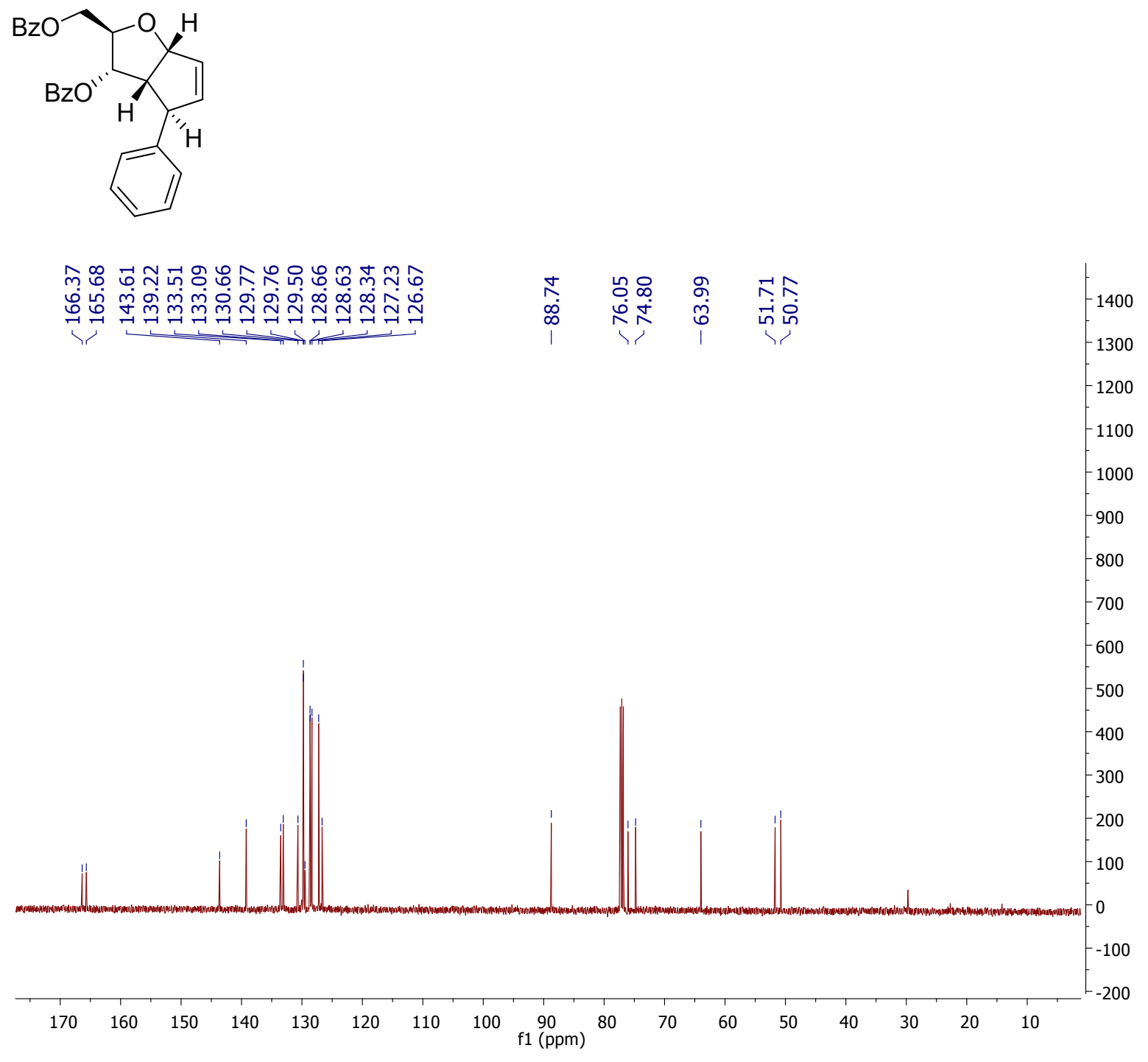


$\underline{1} \mathrm{H}$ NMR (400 MHz, $\mathrm{CDCl}_{3}$ ) of compound 2aa<smiles>CC(=O)OC[C@H]1O[C@@H]2C(O)[C@H](O)[C@@H](c3cccc(C)c3)[C@H]2[C@H]1OC(C)=O</smiles>

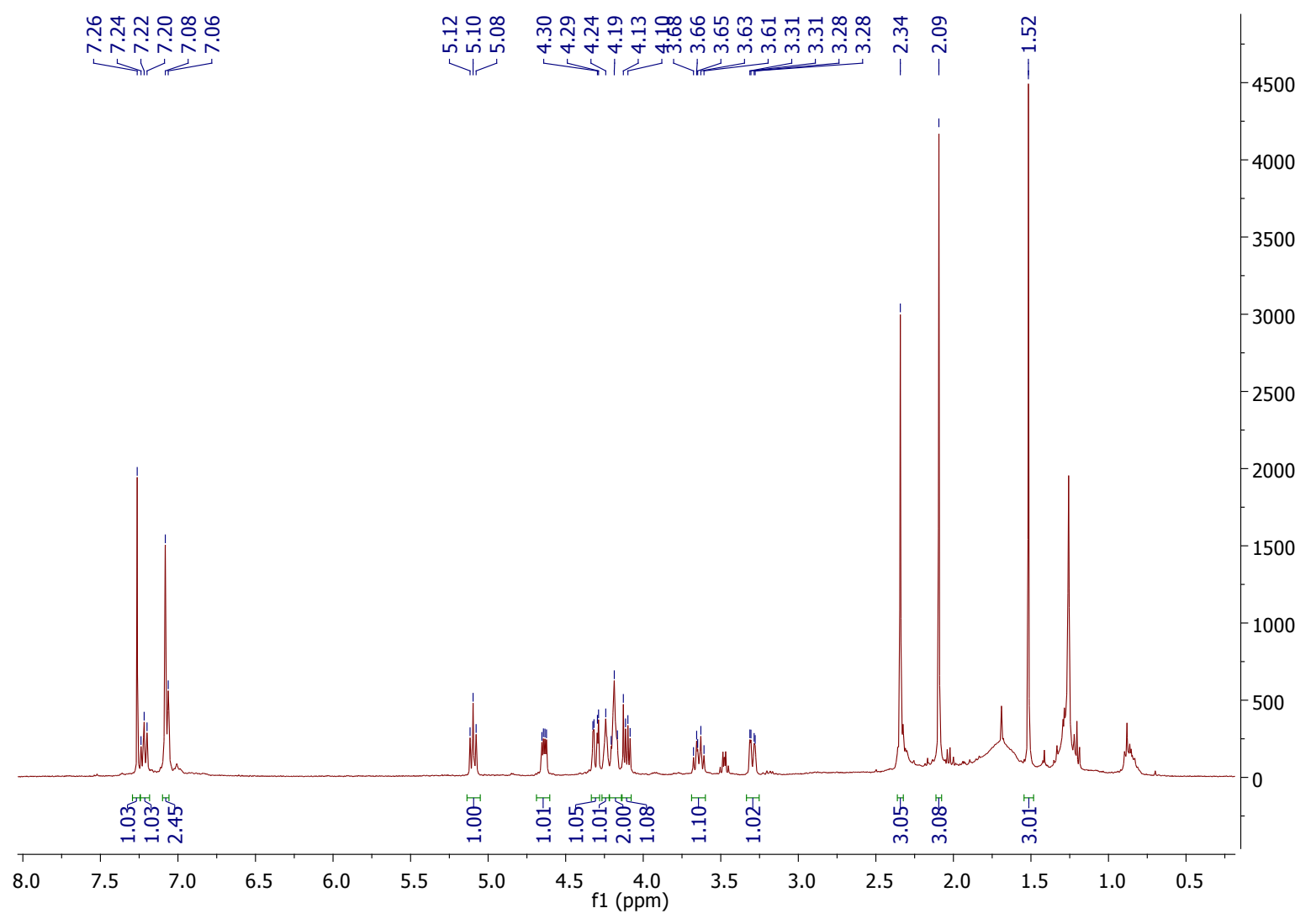


$\underline{{ }^{13} \mathrm{C} \text { NMR (101 MHz, } \mathrm{CDCl}_{3} \text { ) of compound 2aa }}$

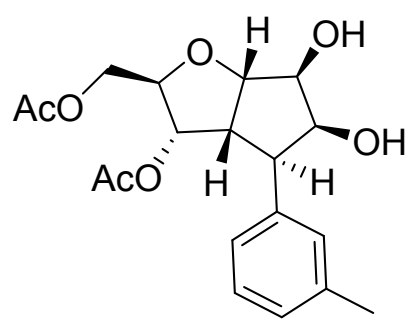

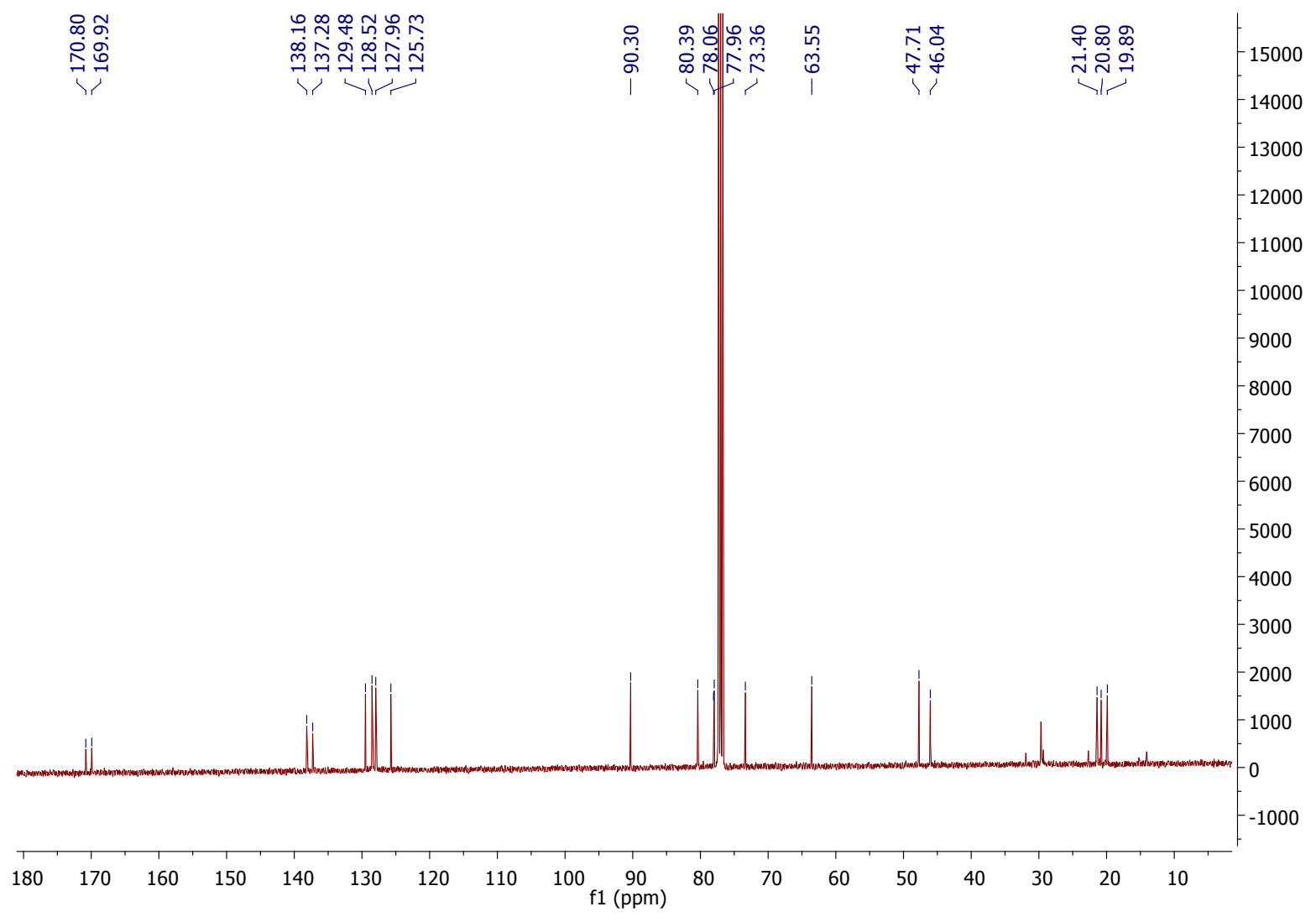




\section{$\underline{{ }^{1} \mathrm{H} \text { NMR (400 MHz, } \mathrm{CDCl}_{3} \text { ) of compound } 6}$}<smiles>CC(=O)OC[C@H]1OC(O)C=C[C@@H]1OC(C)=O</smiles>

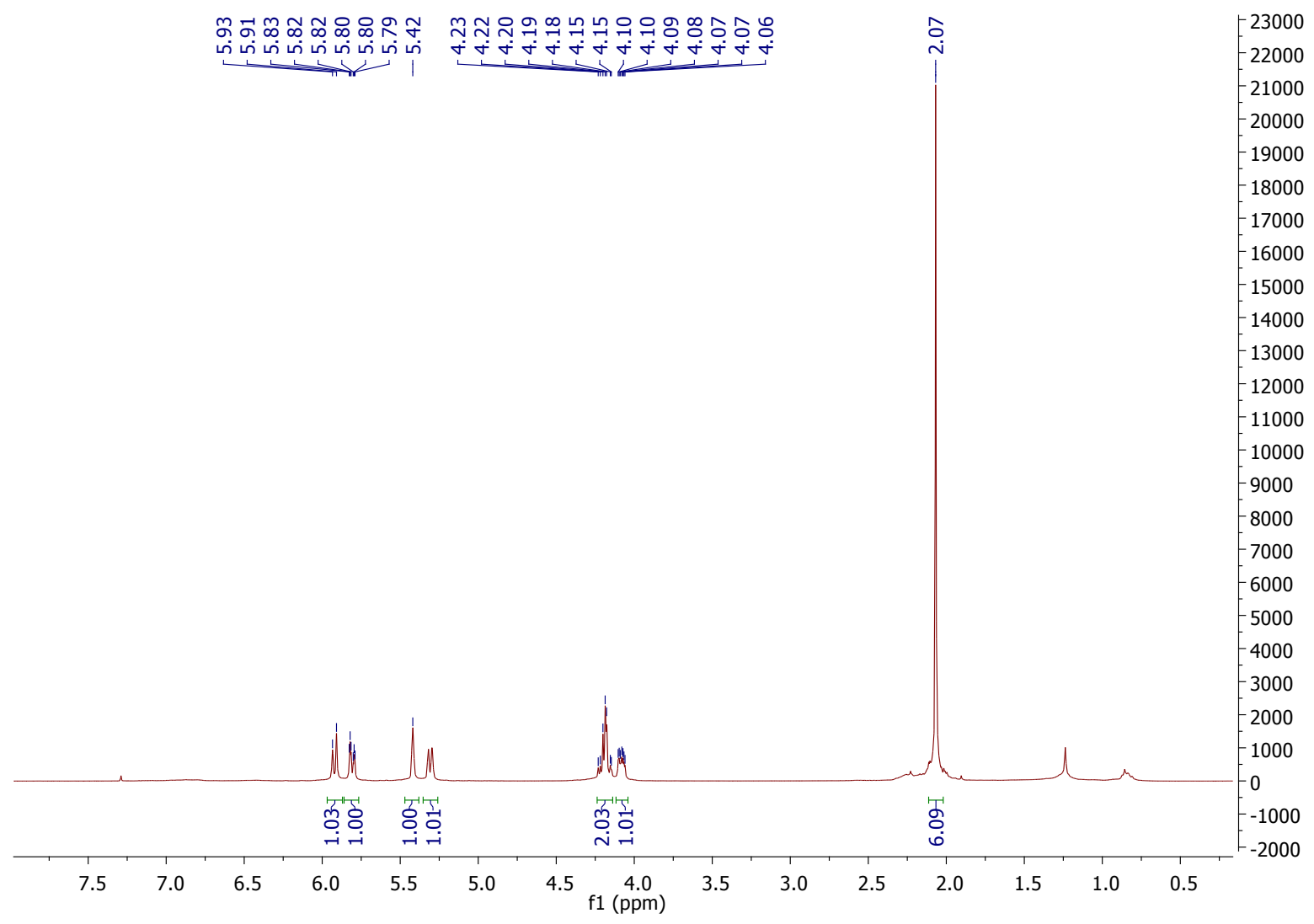




\section{${ }^{13} \mathrm{C}$ NMR (101 MHz, $\mathrm{CDCl}_{3}$ ) of compound 6}<smiles>CC(=O)OC[C@H]1OC(O)C=C[C@@H]1OC(C)=O</smiles>

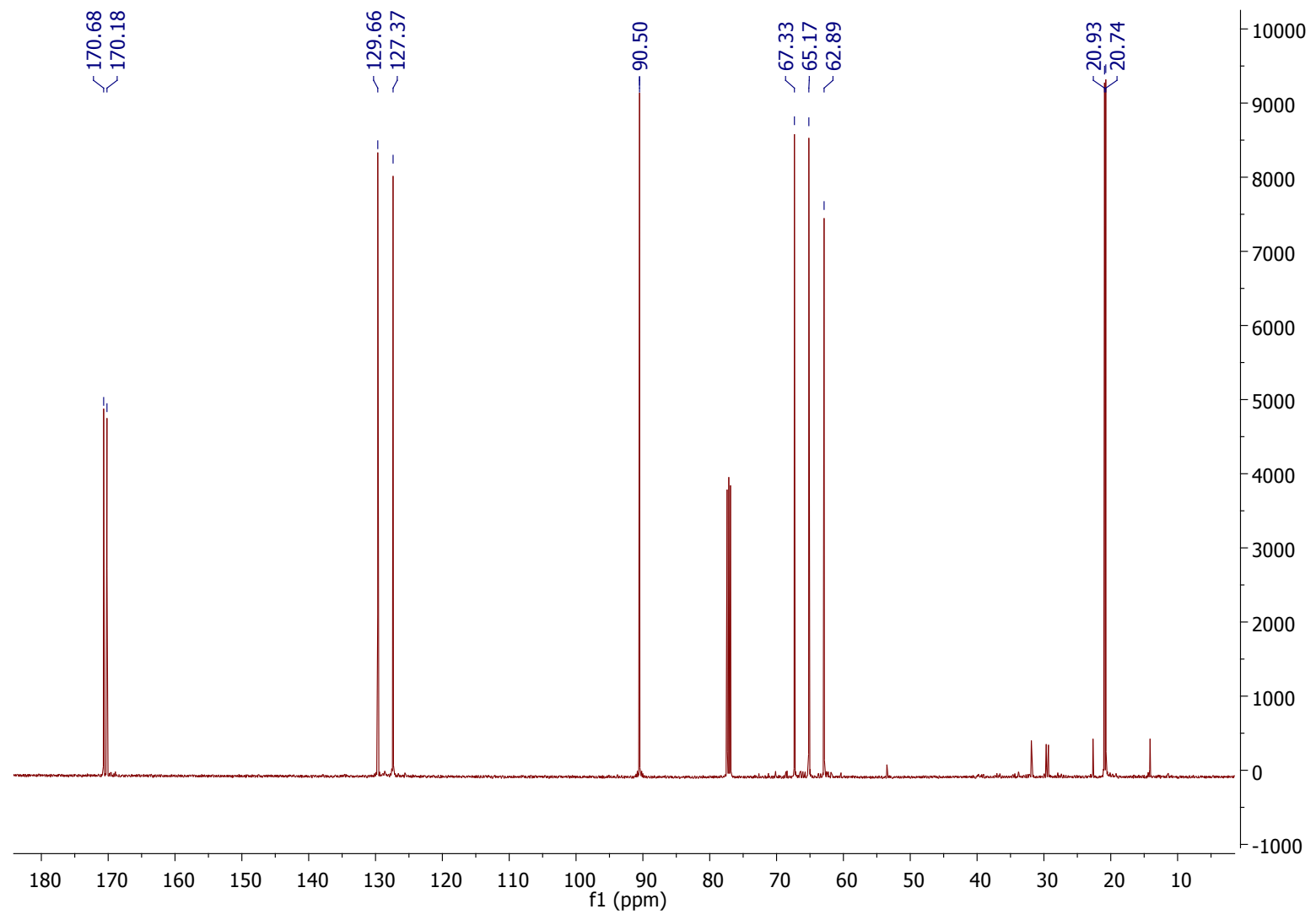


NOESY of 2:
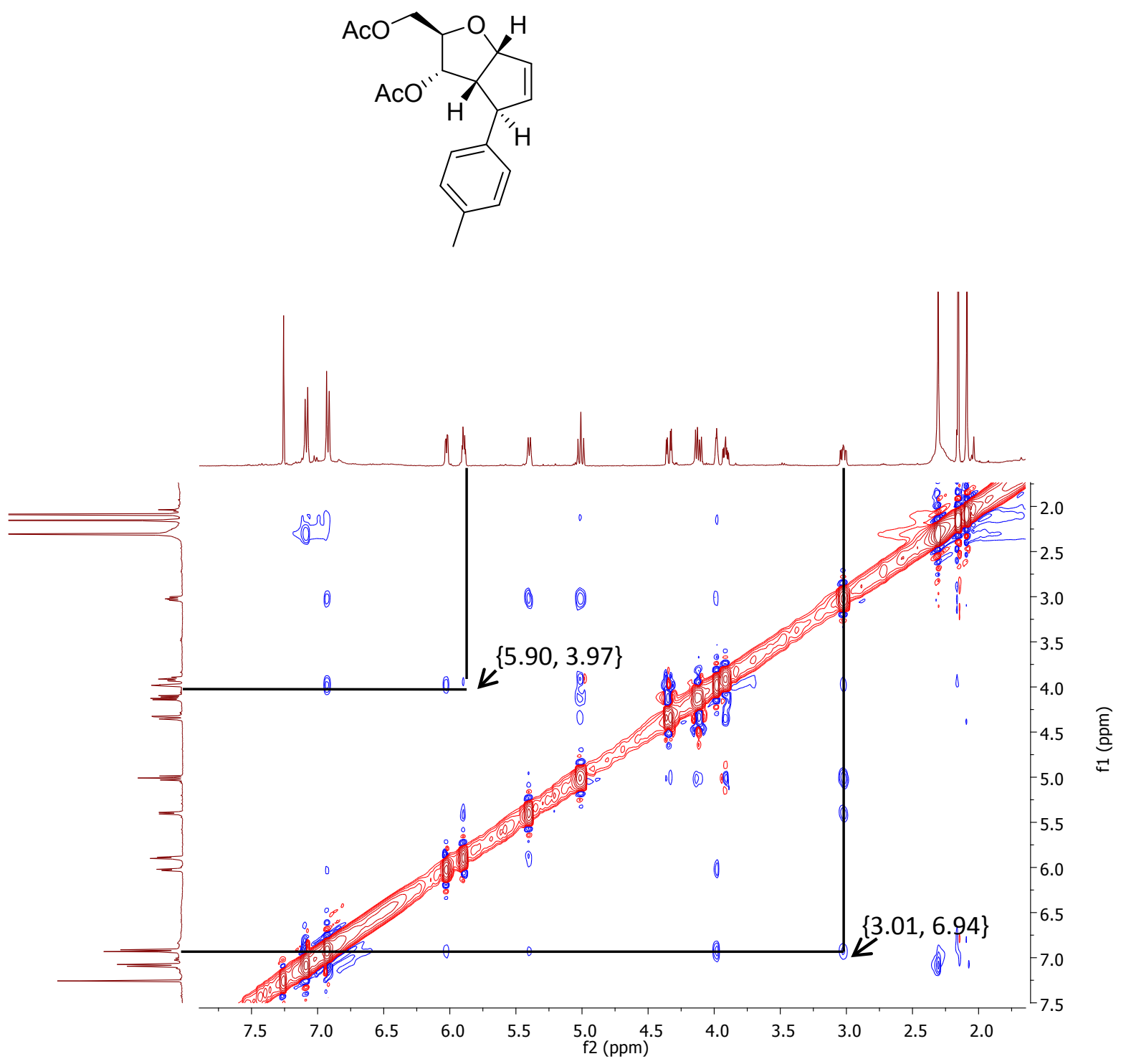

$\mathrm{H} 2-3.01, \mathrm{H} 6-5.90, \mathrm{H} 8-3.97, \mathrm{H} 13-6.94$ 


\section{COSY of 2:}

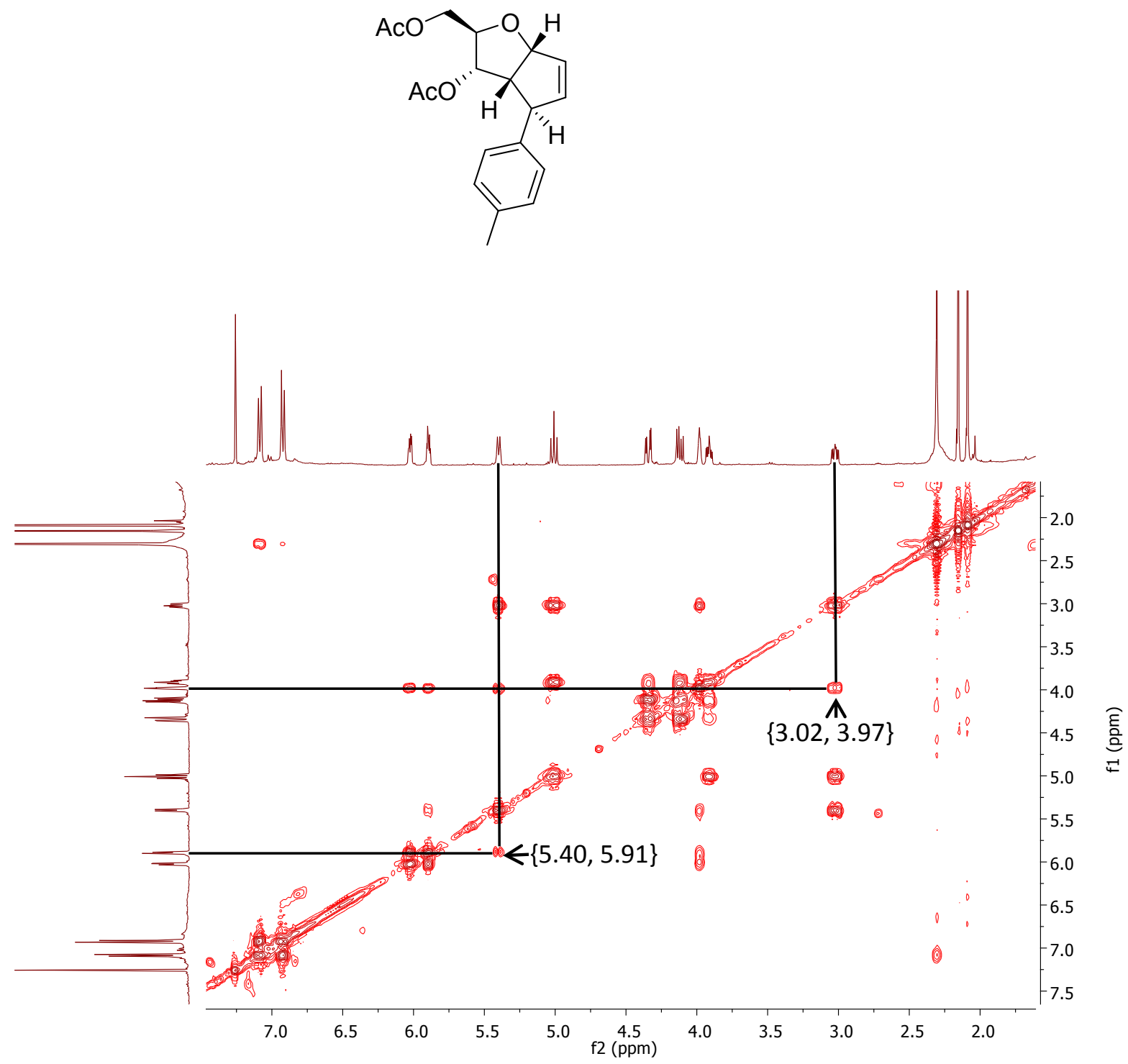

$\mathrm{H} 1-5.40, \mathrm{H} 2-3.02, \mathrm{H} 6-5.91, \mathrm{H} 8-3.97$ 
HSQC of 2:
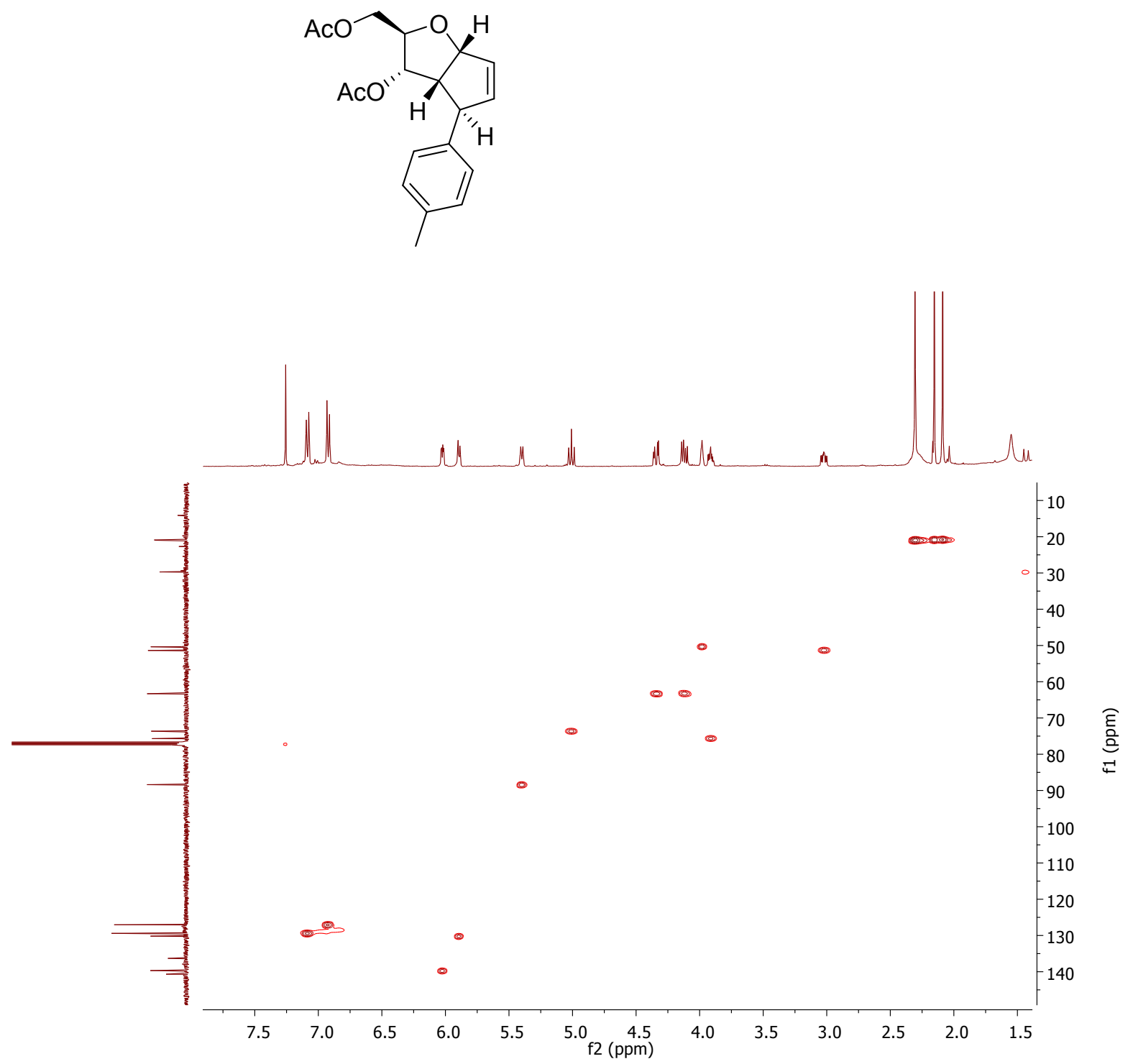
HMBC of 2:
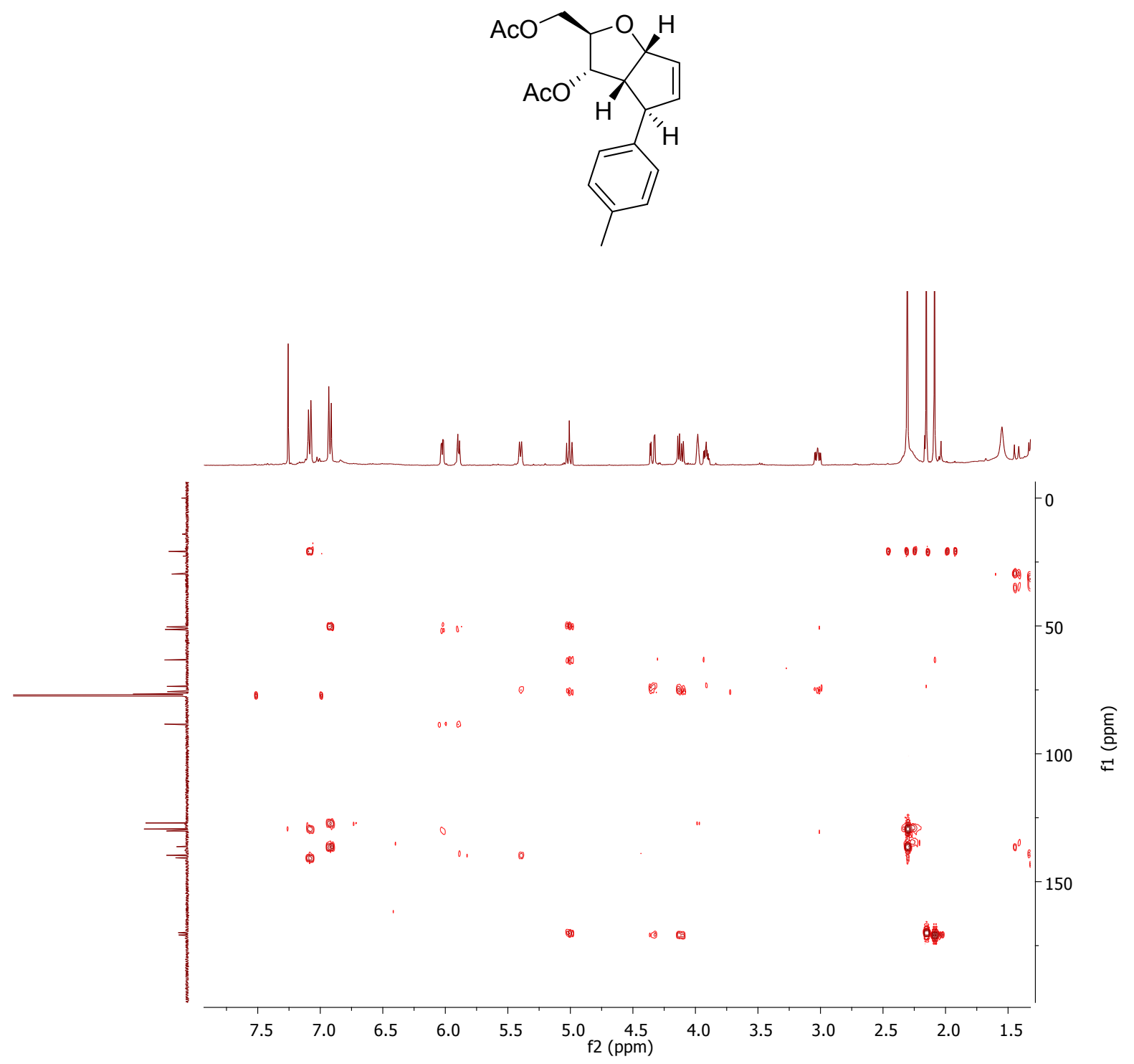
NOESY of 2aa;
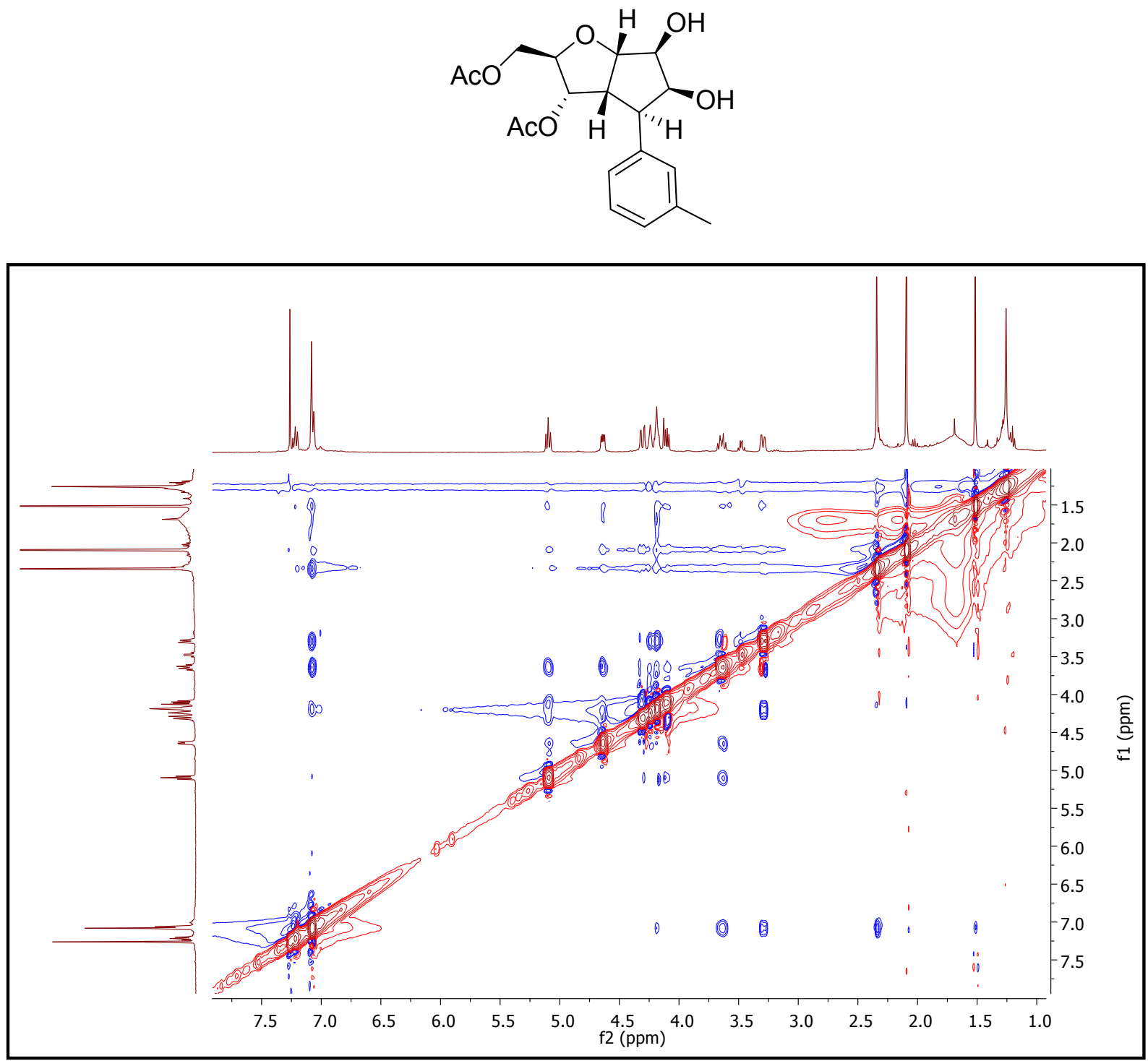
COSY of 2aa:
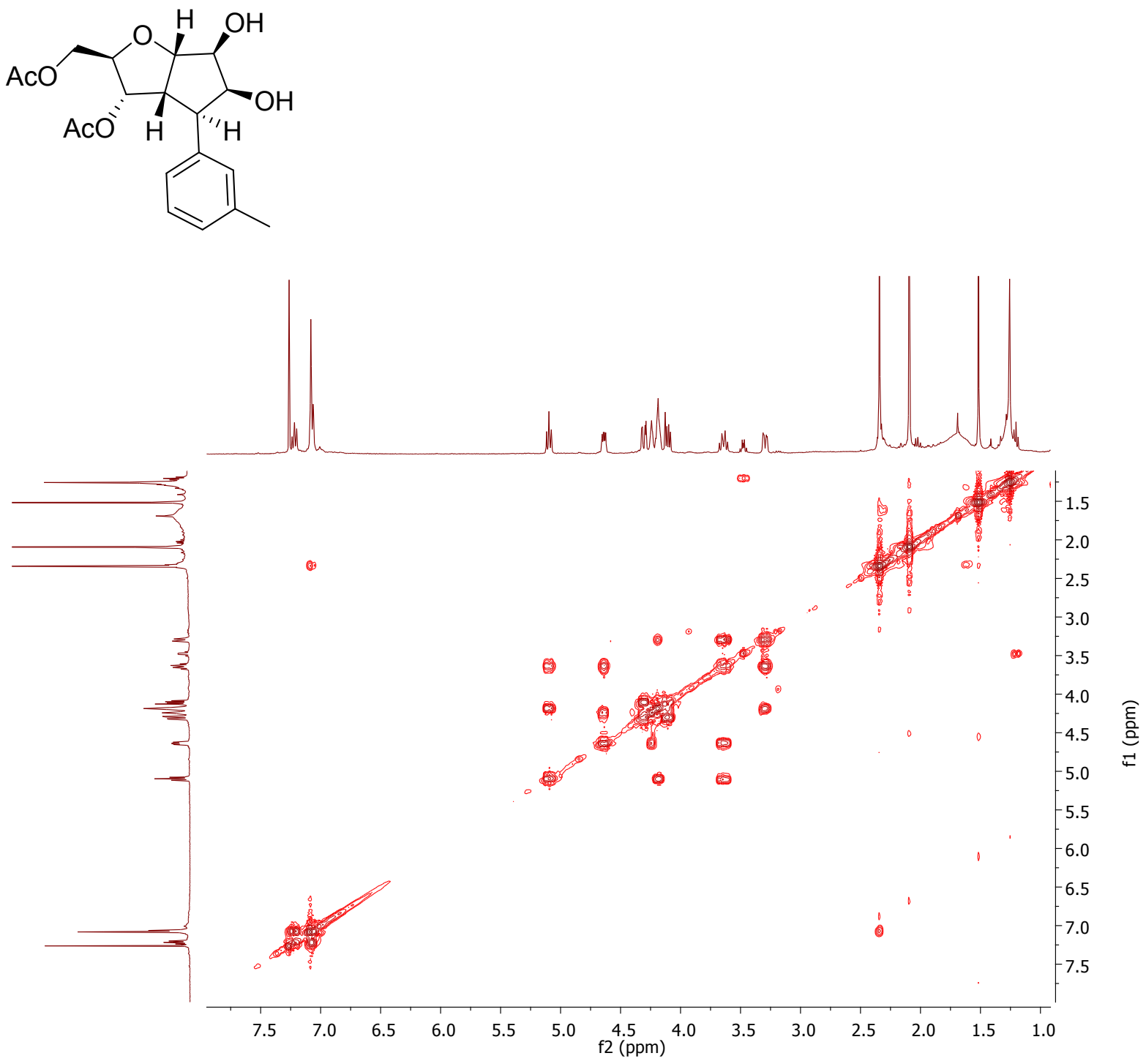
HSQC of 2aa:<smiles></smiles>

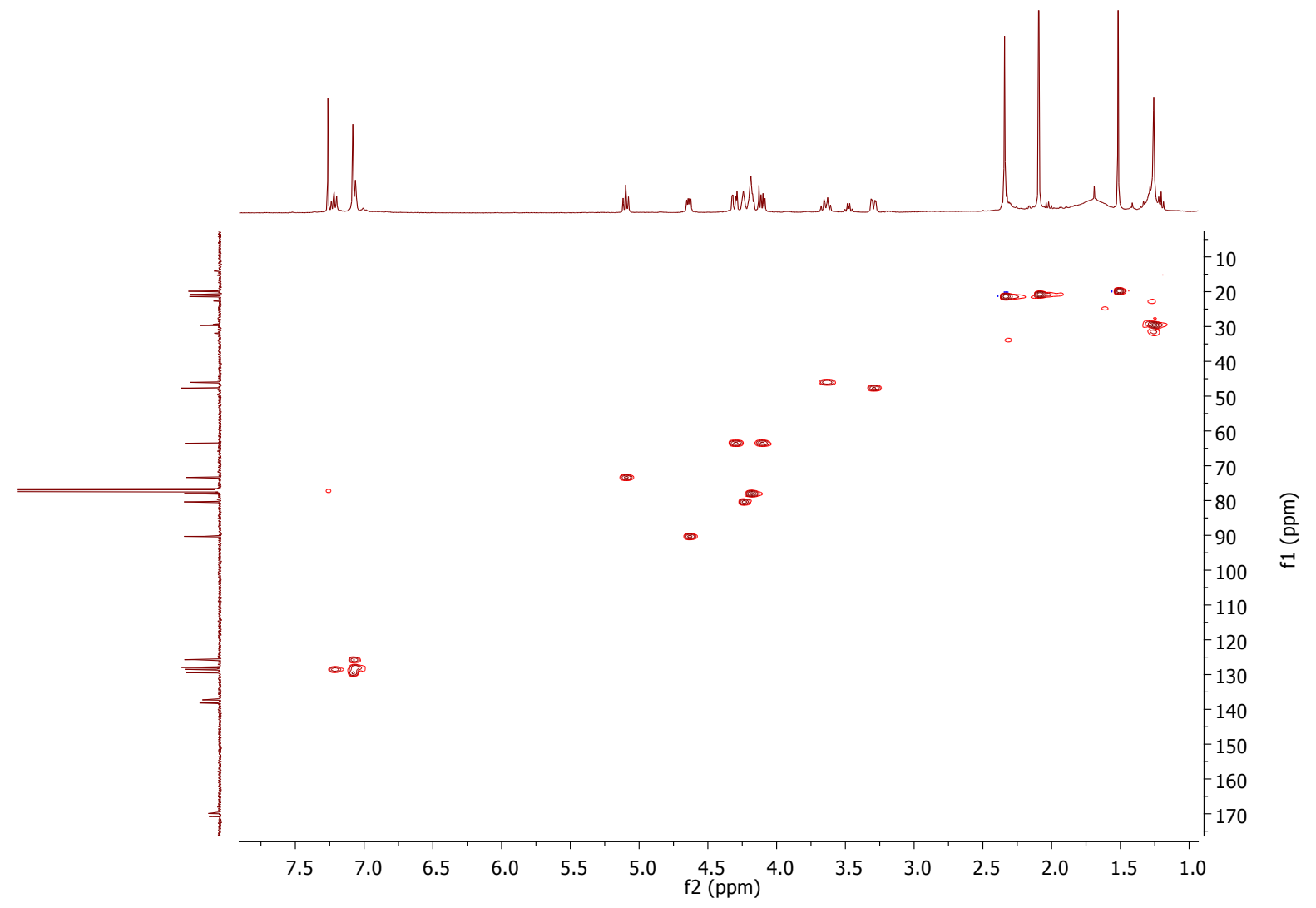


HMBC of 2aa:<smiles>CC(=O)OC[C@H]1O[C@@H]2[C@@H](O)C(O)[C@H](c3cccc(C)c3)[C@H]2[C@H]1OC(C)C</smiles>

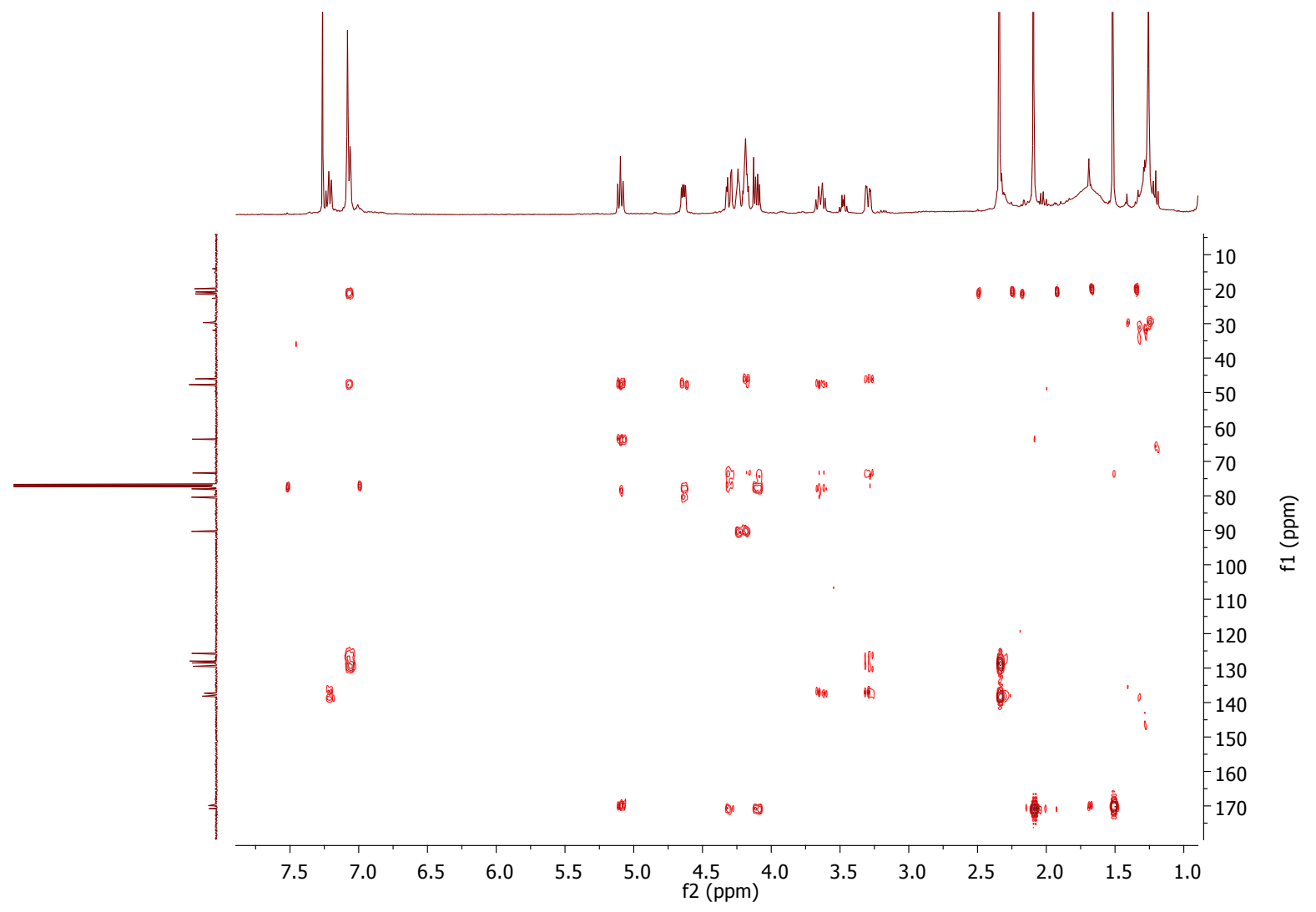




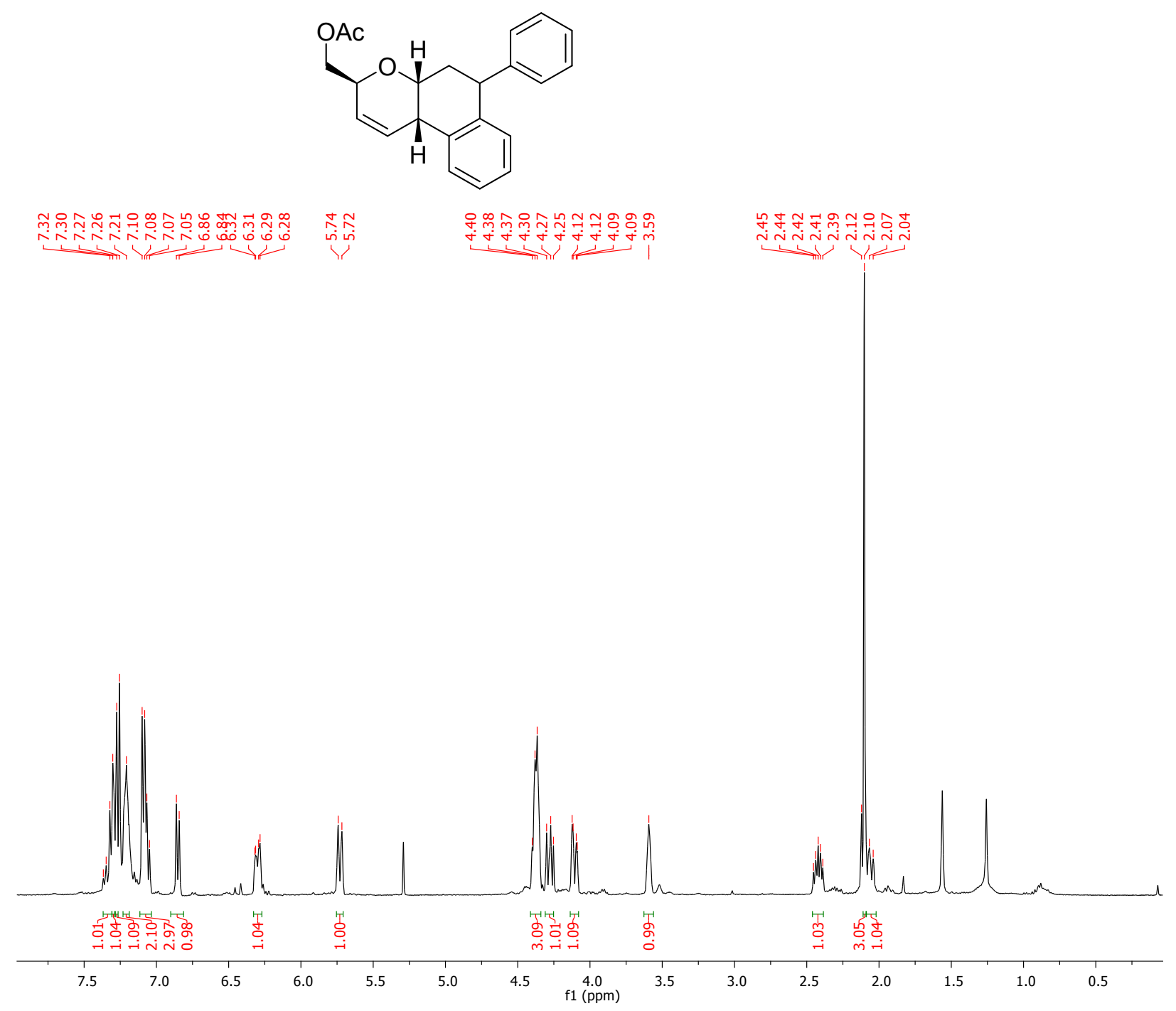


$\underline{{ }^{13} \mathrm{C} \text { NMR (101 MHz, } \mathrm{CDCl}_{3} \text { ) of compound } 7}$

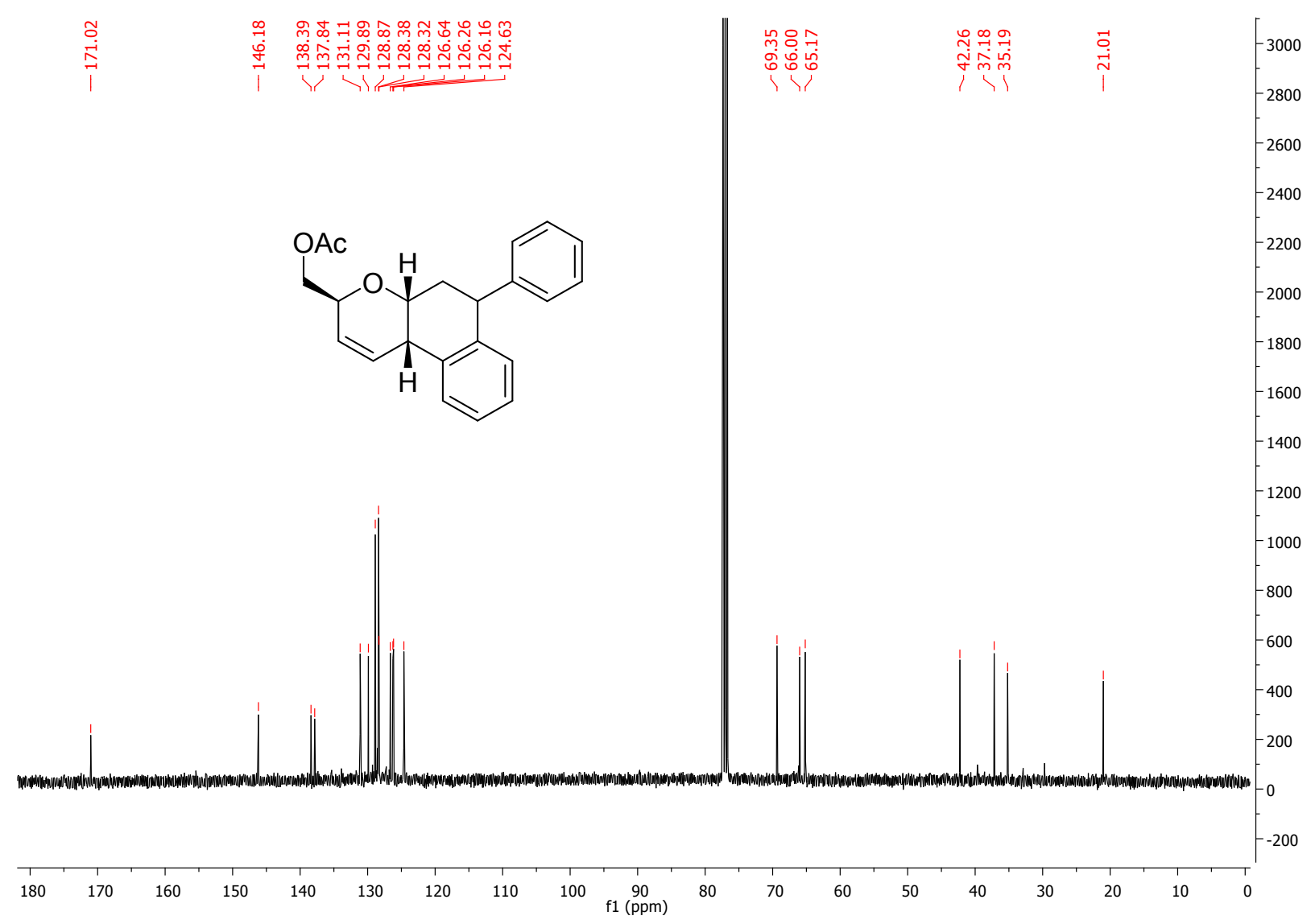




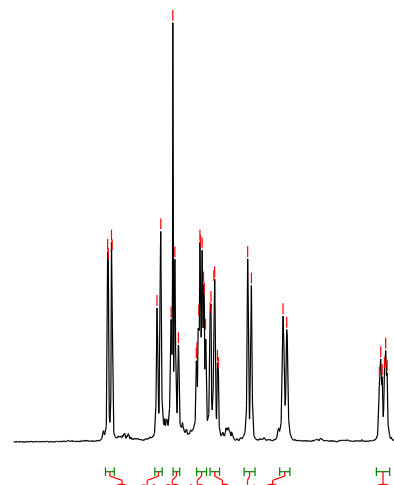<smiles>CC(=O)OCC1C=CC2CC(c3ccccc3Br)CC2O1</smiles>

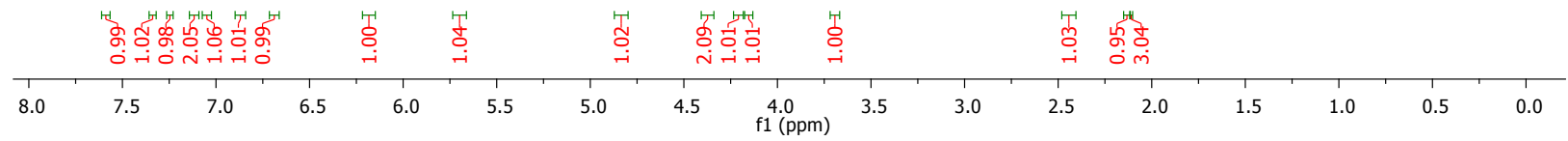


${ }^{13} \mathrm{C}$ NMR (101 MHz, $\mathrm{CDCl}_{3}$ ) of compound 7a

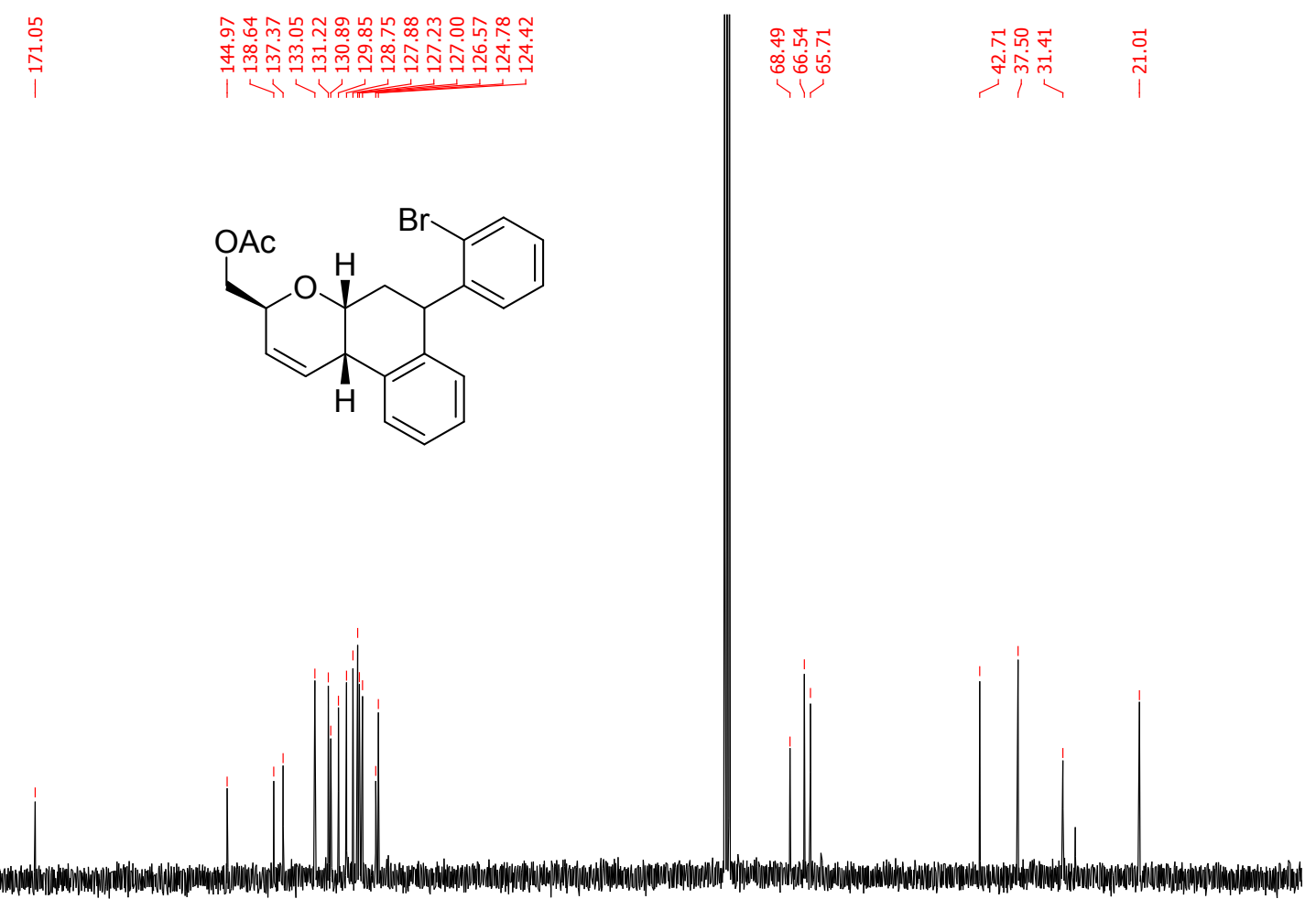

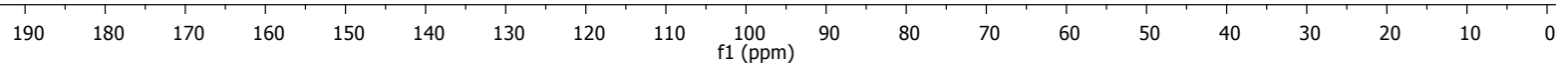




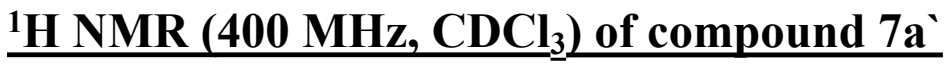

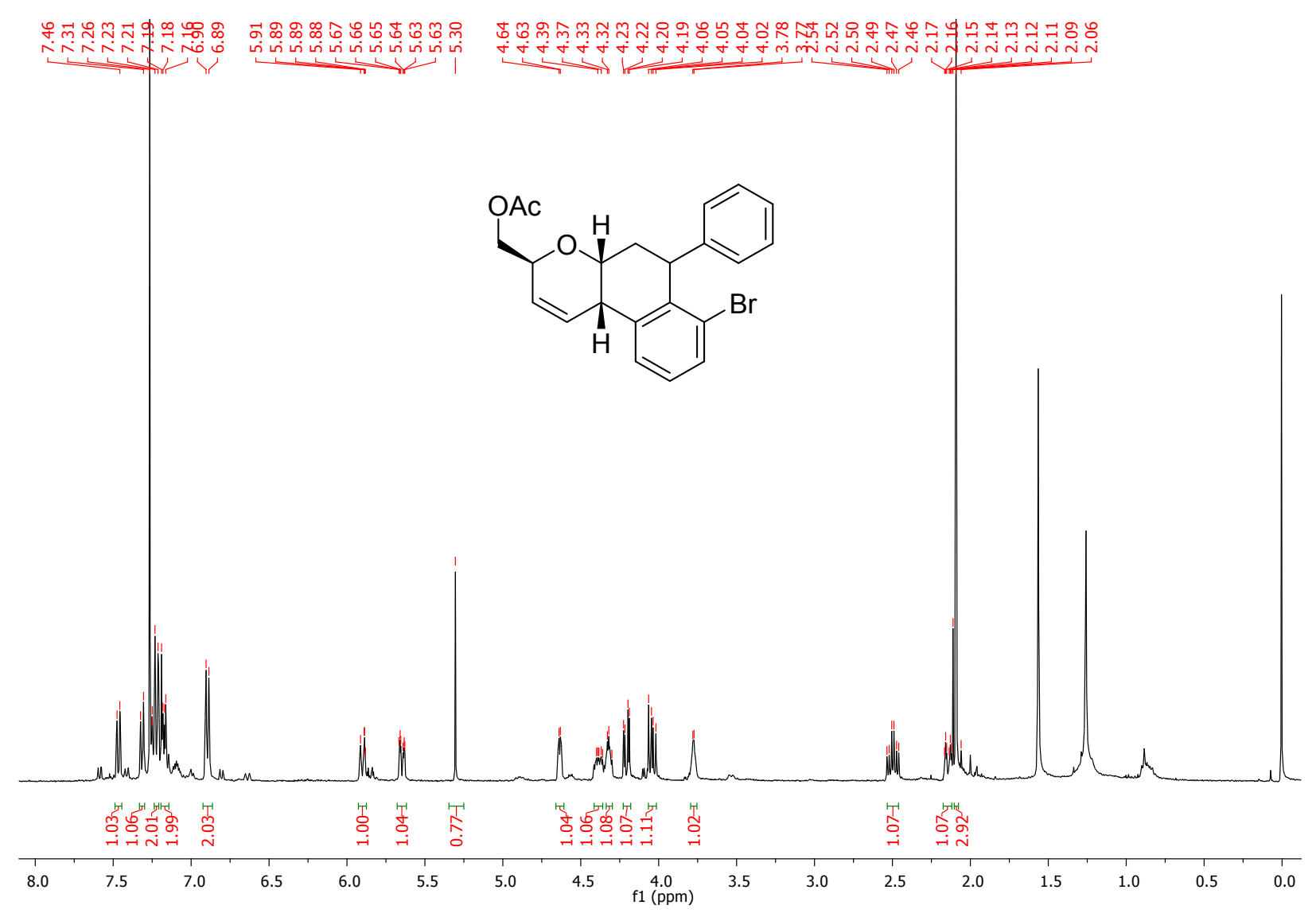


${ }^{13} \mathrm{C}$ NMR (101 MHz, $\mathrm{CDCl}_{3}$ ) of compound $7 \mathrm{a}^{\text {' }}$
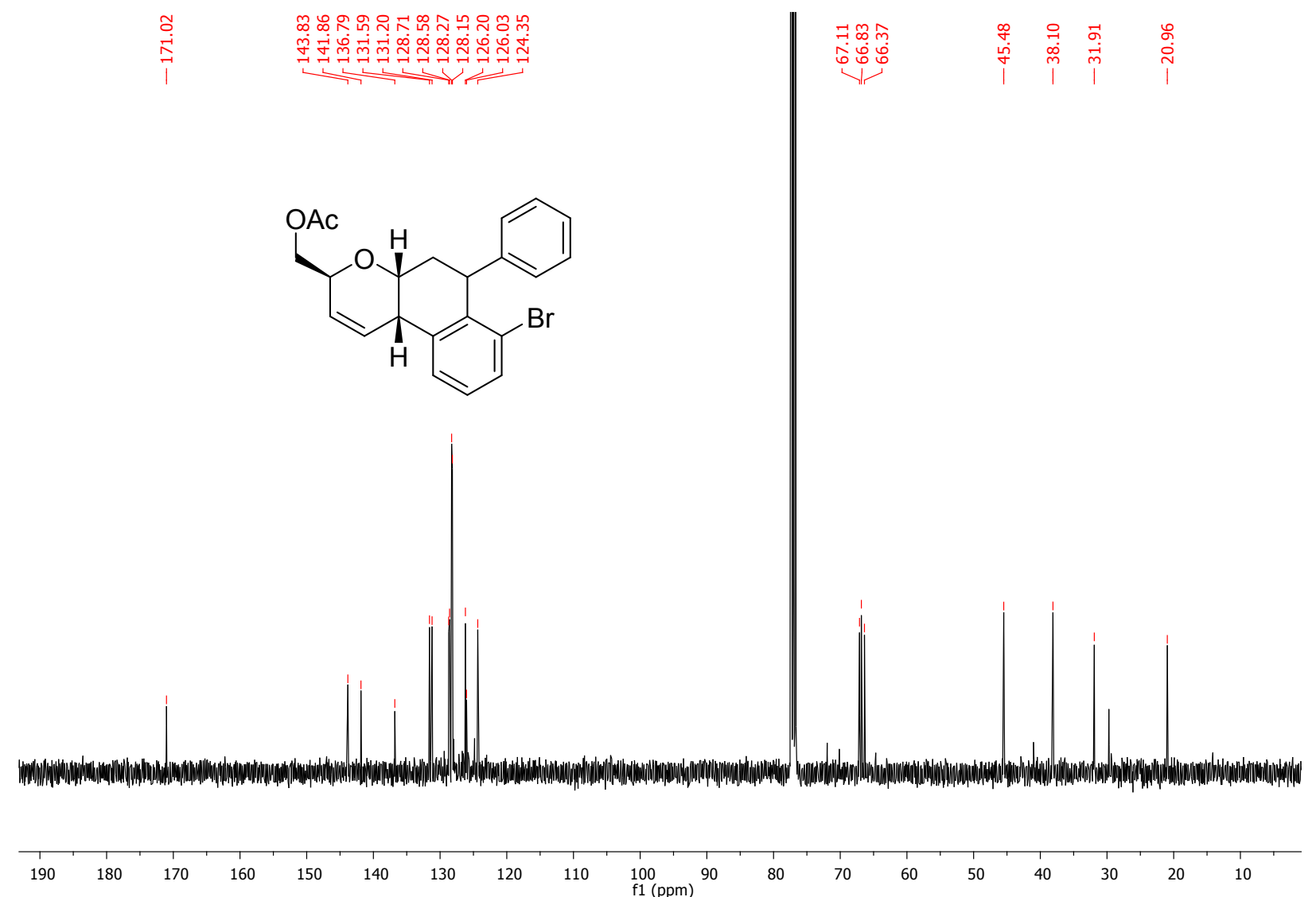


\section{$\underline{\left.{ }^{1} \mathrm{H} \text { NMR (400 MHz, } \mathrm{CDCl}_{3}\right) \text { of compound } 7 \mathrm{~b}}$}

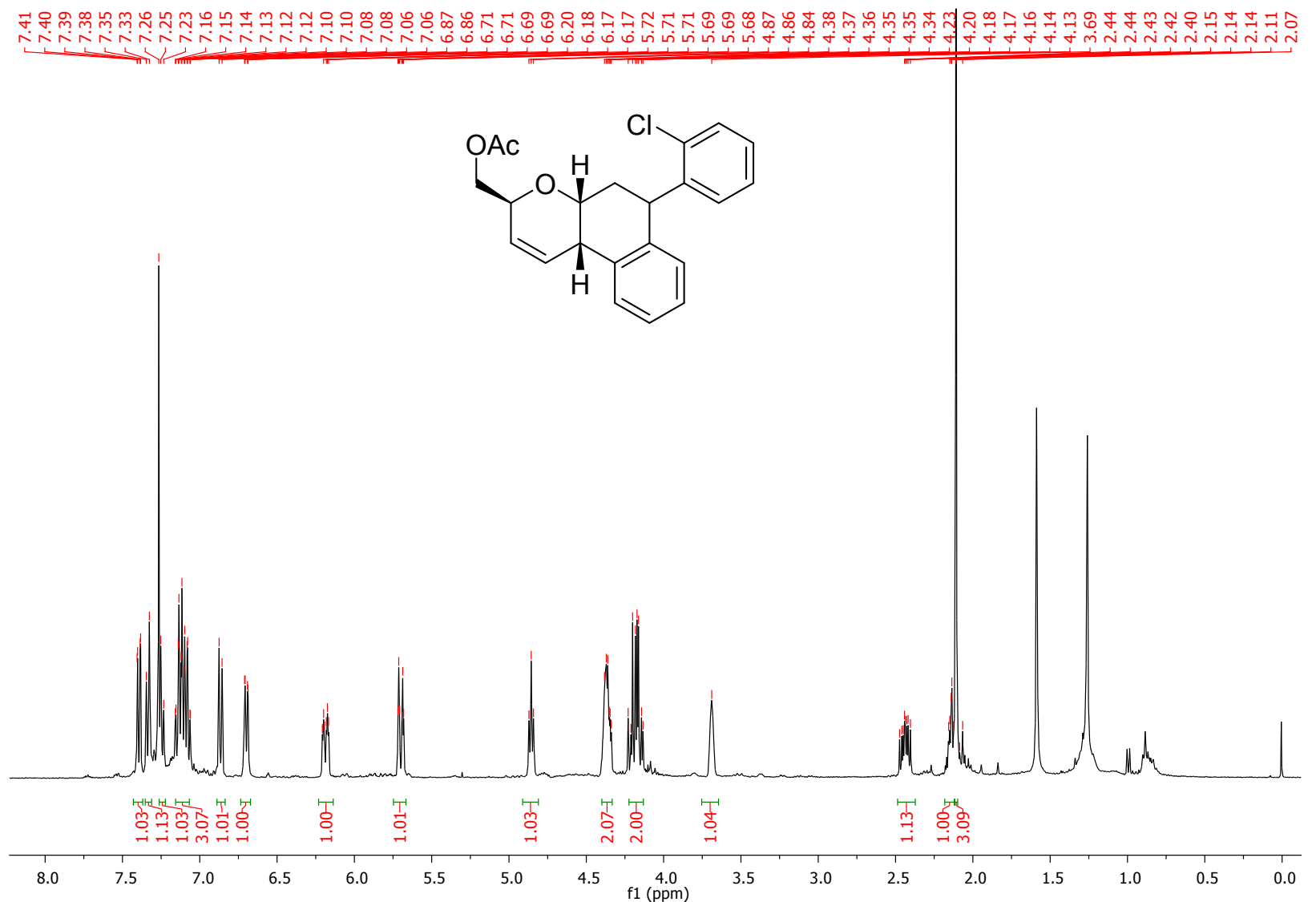


${ }^{13} \mathrm{C}$ NMR (101 MHz, $\left.\mathrm{CDCl}_{3}\right)$ of compound $7 \mathrm{~b}$

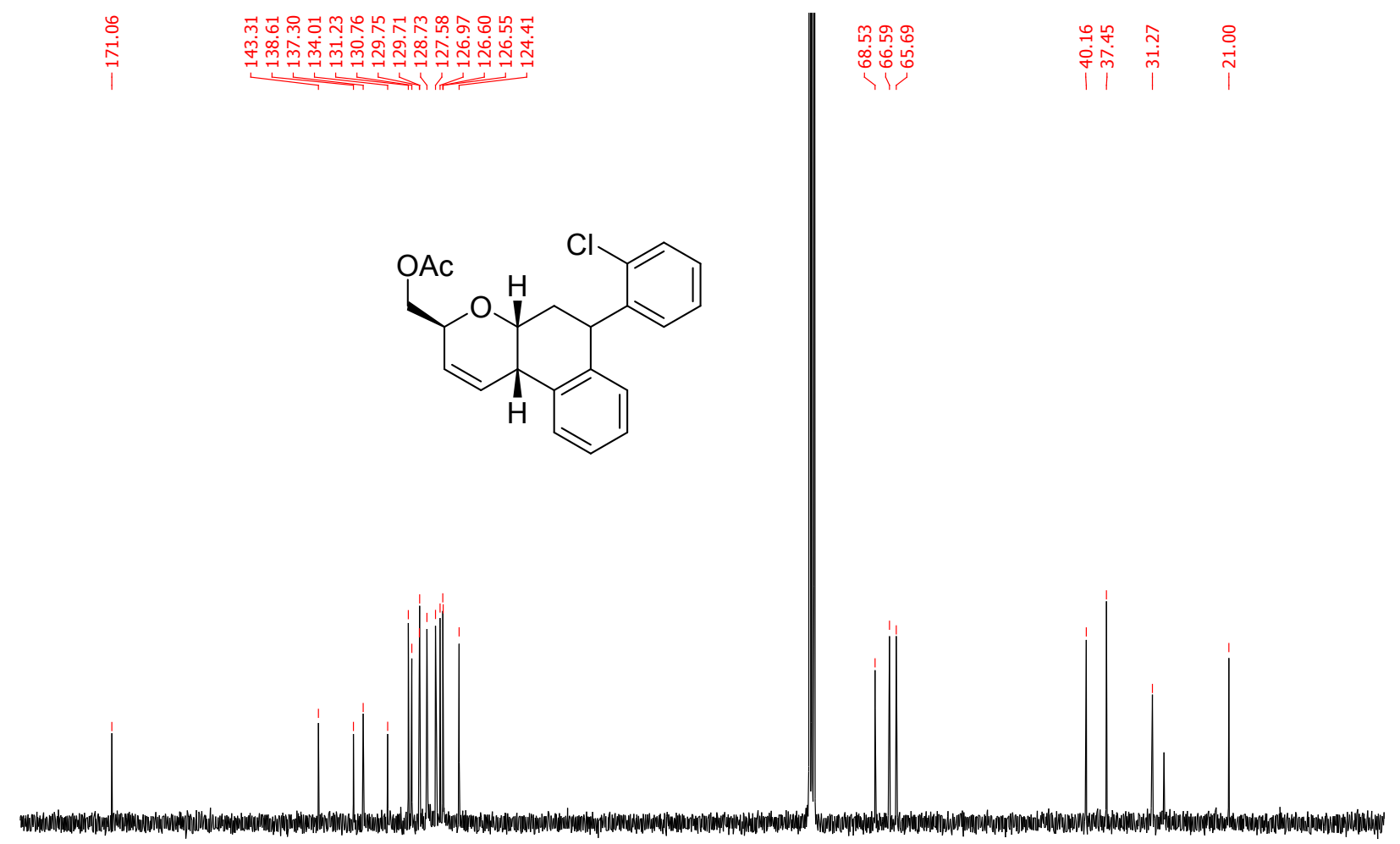

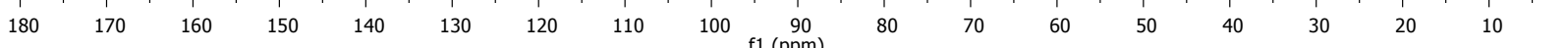




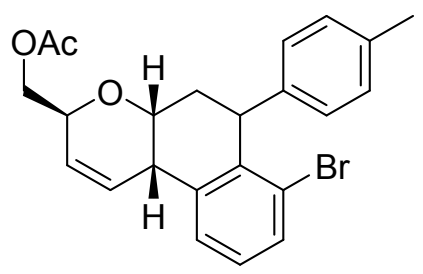

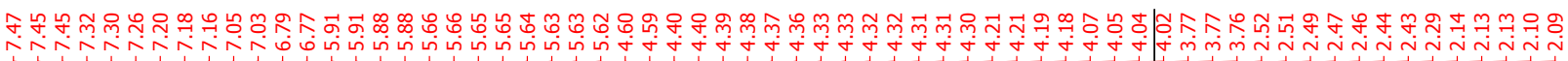

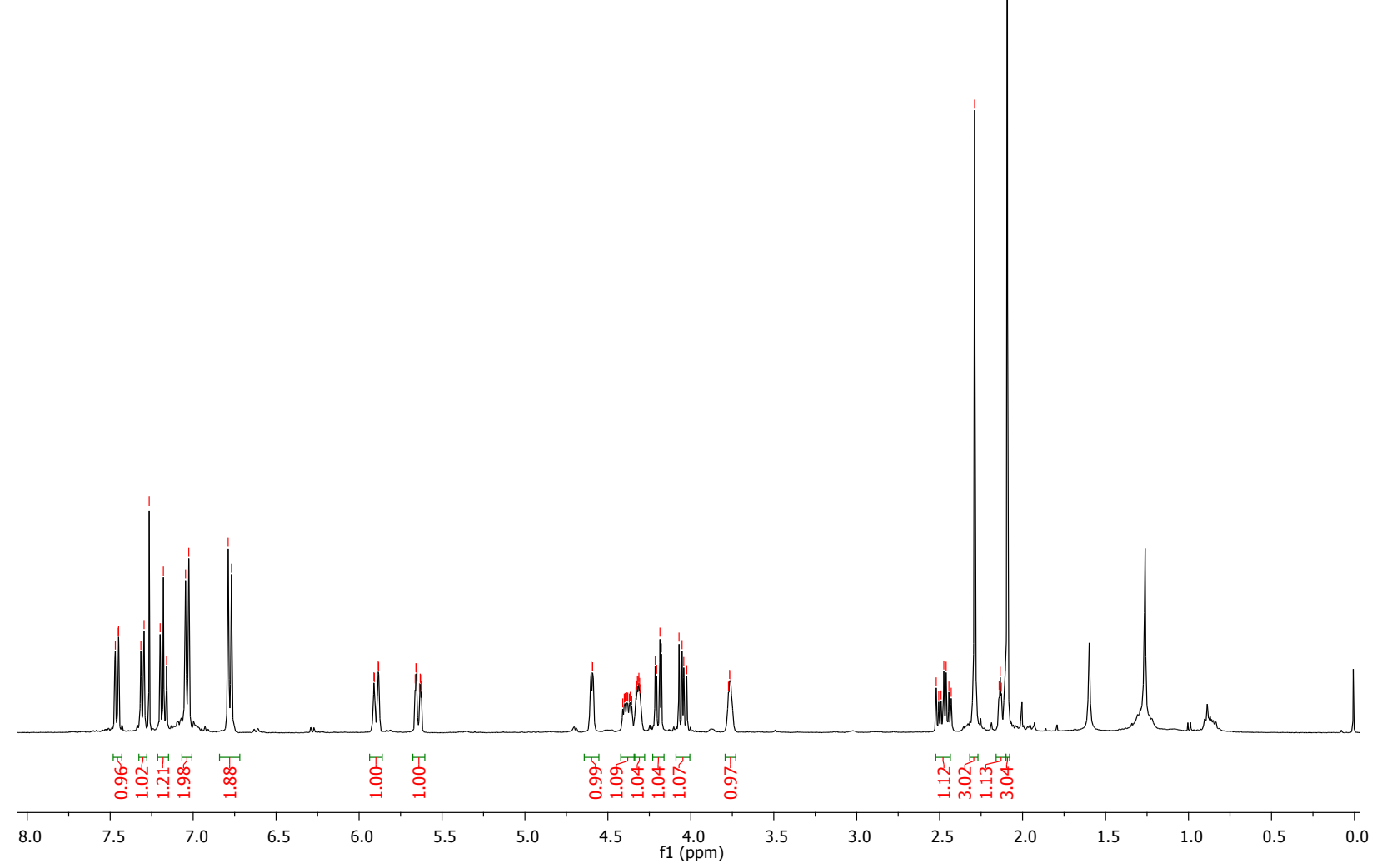




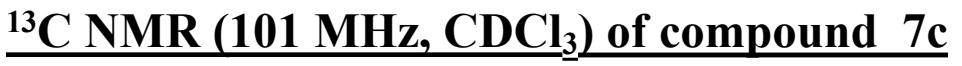<smiles>CC(=O)CC1C=C[C@H]2c3cccc(Br)c3C(c3ccc(C)cc3)C[C@H]2O1</smiles>

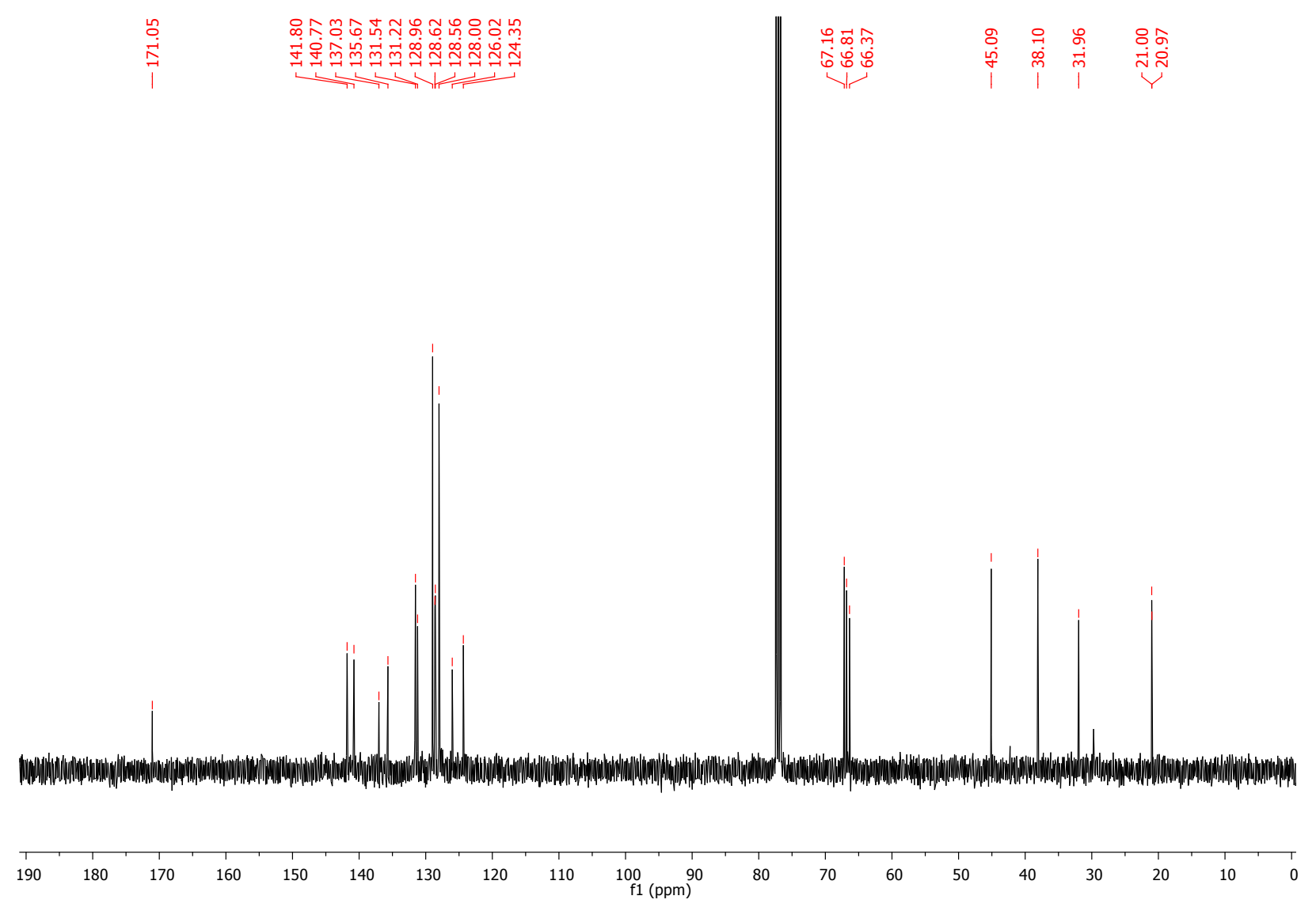

$\underline{{ }^{1} \mathrm{H} N M R}$ (400 MHz, $\mathrm{CDCl}_{3}$ ) of compound 7d 

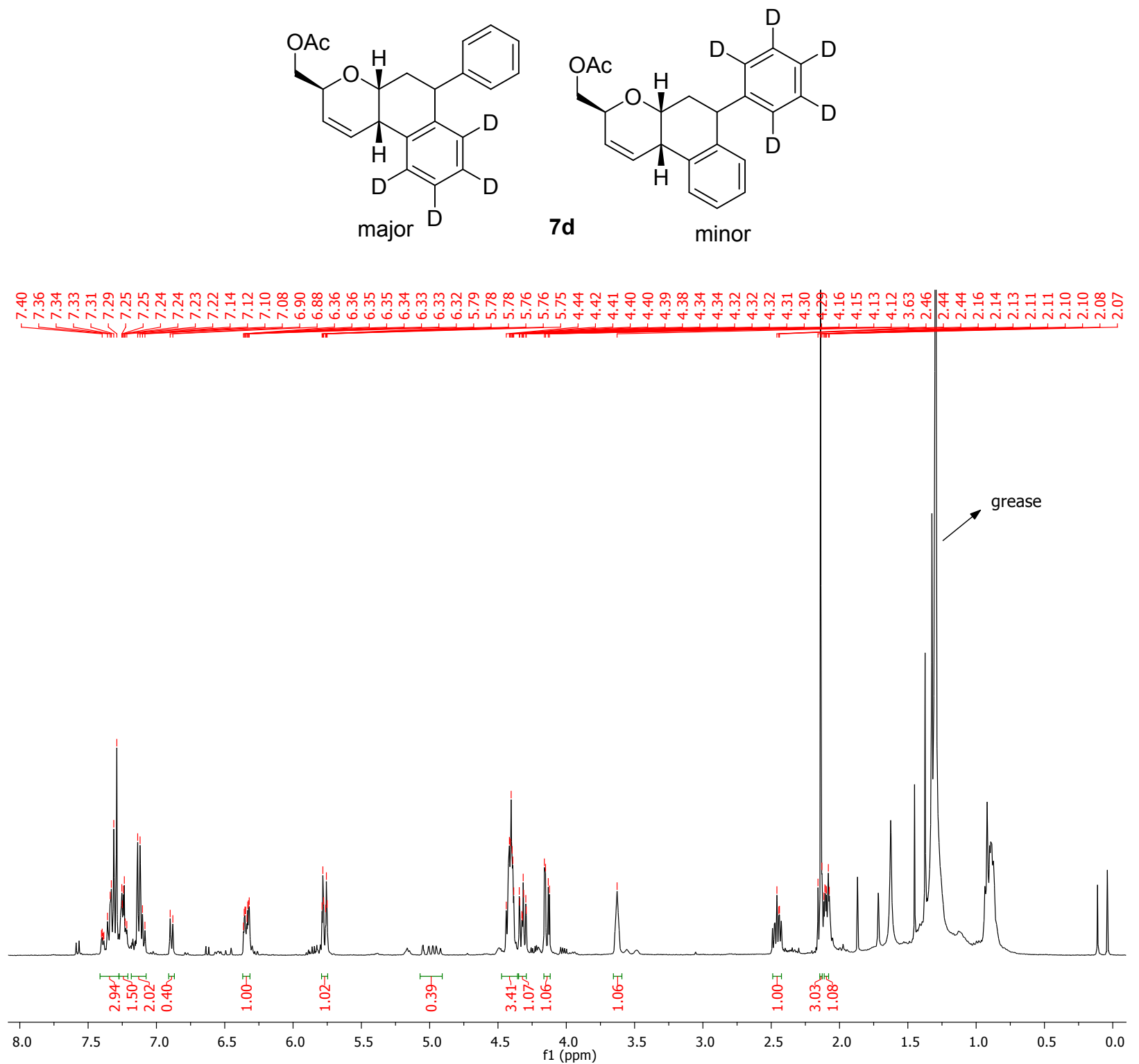

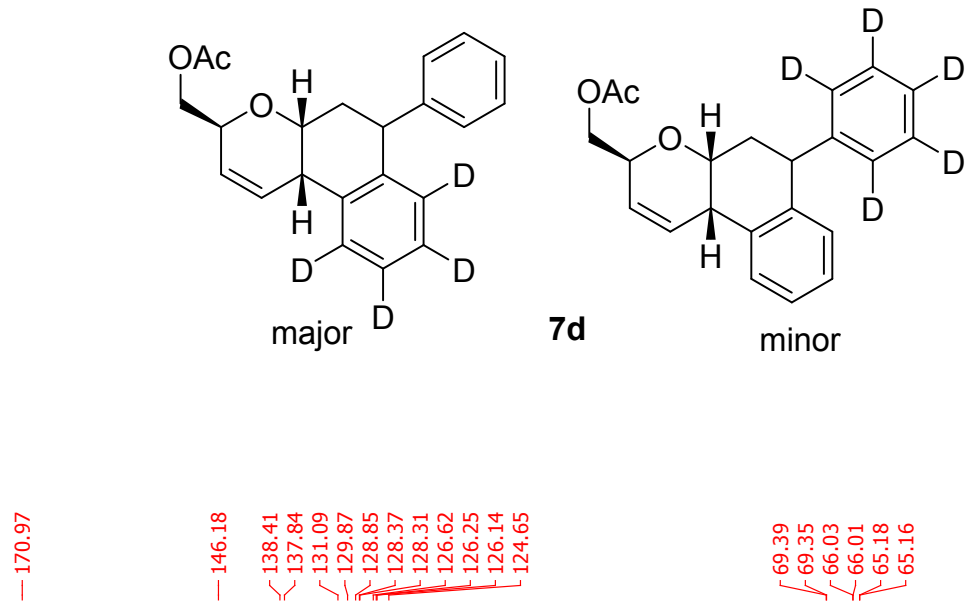

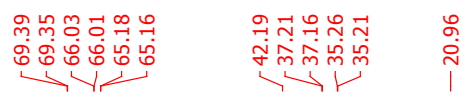

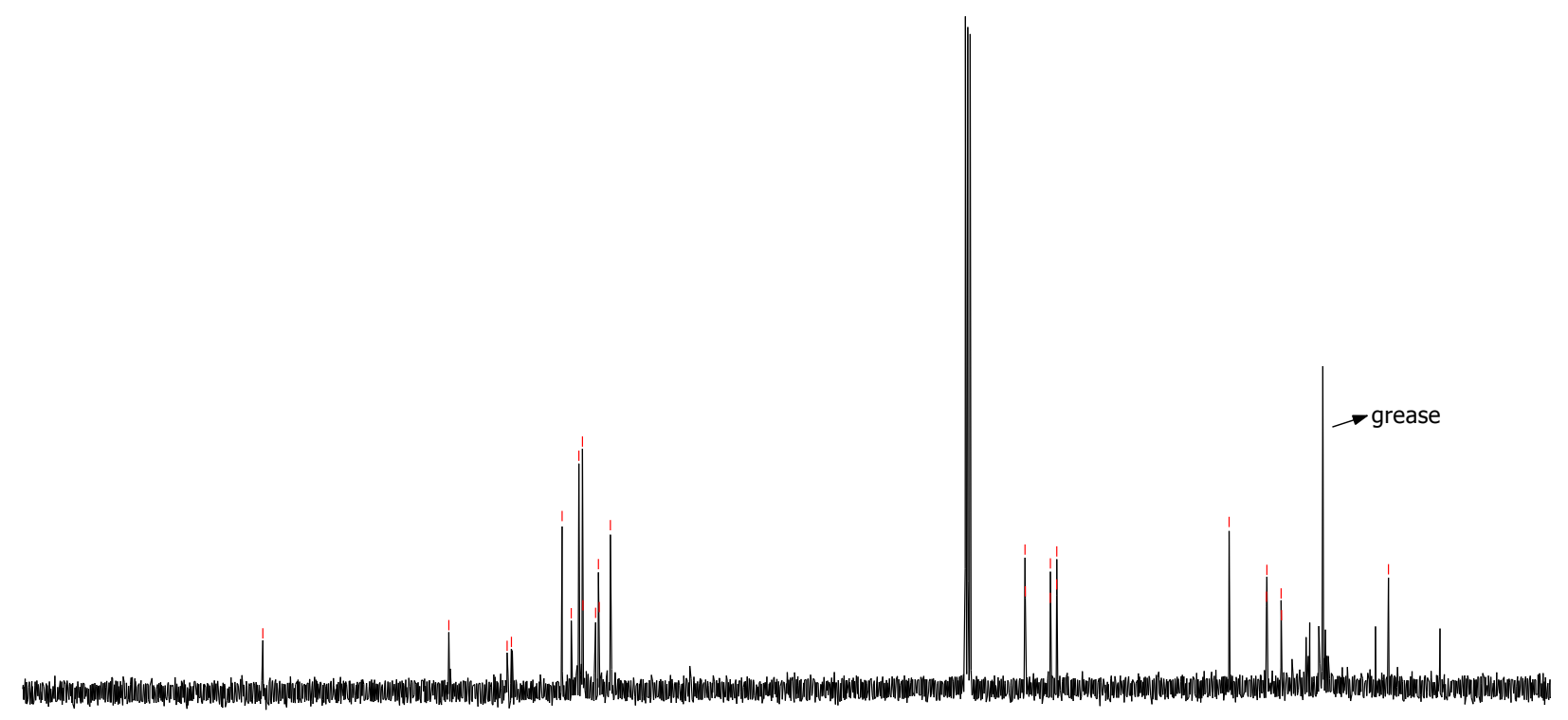

$\begin{array}{lllllllllllllllllllll}200 & 190 & 180 & 170 & 160 & 150 & 140 & 130 & 120 & 110 & \begin{array}{c}100 \\ (100)\end{array} & 90 & 80 & 70 & 60 & 50 & 40 & 30 & 20 & 10 & 0\end{array}$ 
$\underline{{ }^{1} \mathrm{H} \text { NMR (400 MHz, } \mathrm{CDCl}_{3} \text { ) of compound } 7 \mathrm{e}}$

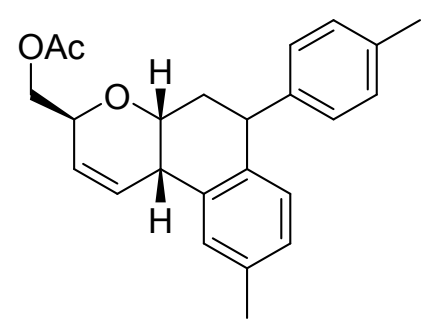

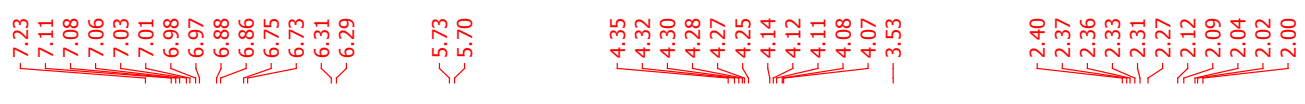

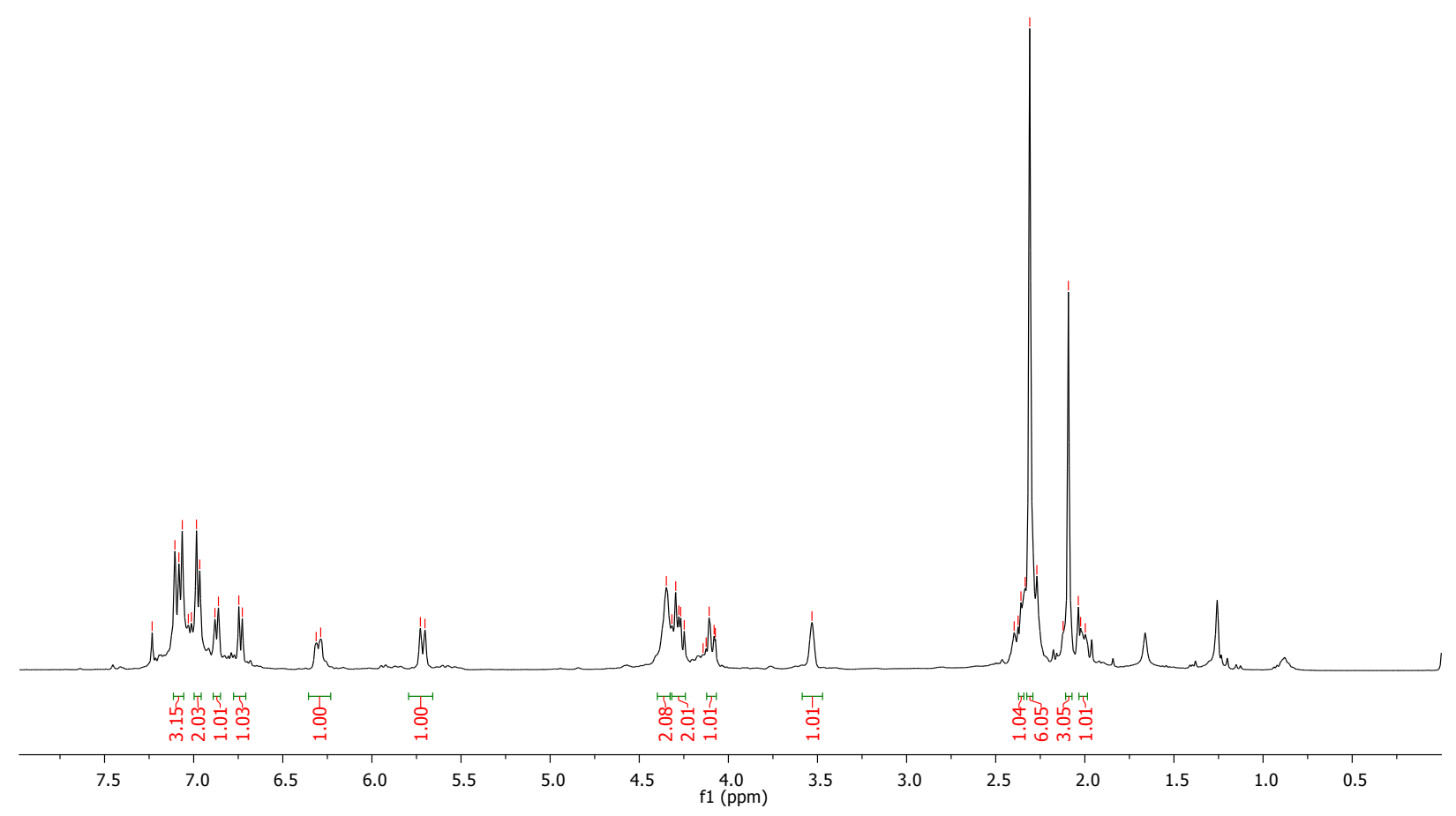

$\underline{\left.{ }^{13} \mathrm{C} \text { NMR (101 MHz, } \mathrm{CDCl}_{3}\right) \text { of compound } 7 \mathrm{e}}$ 


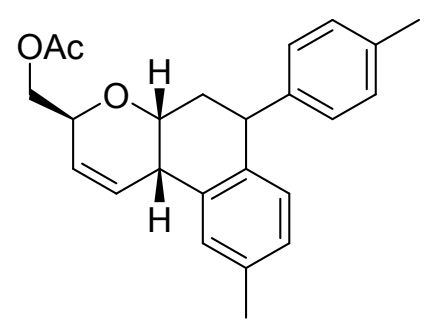

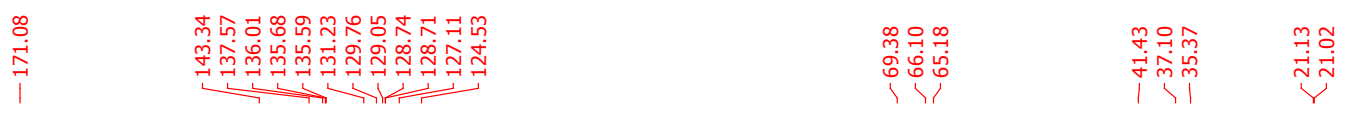

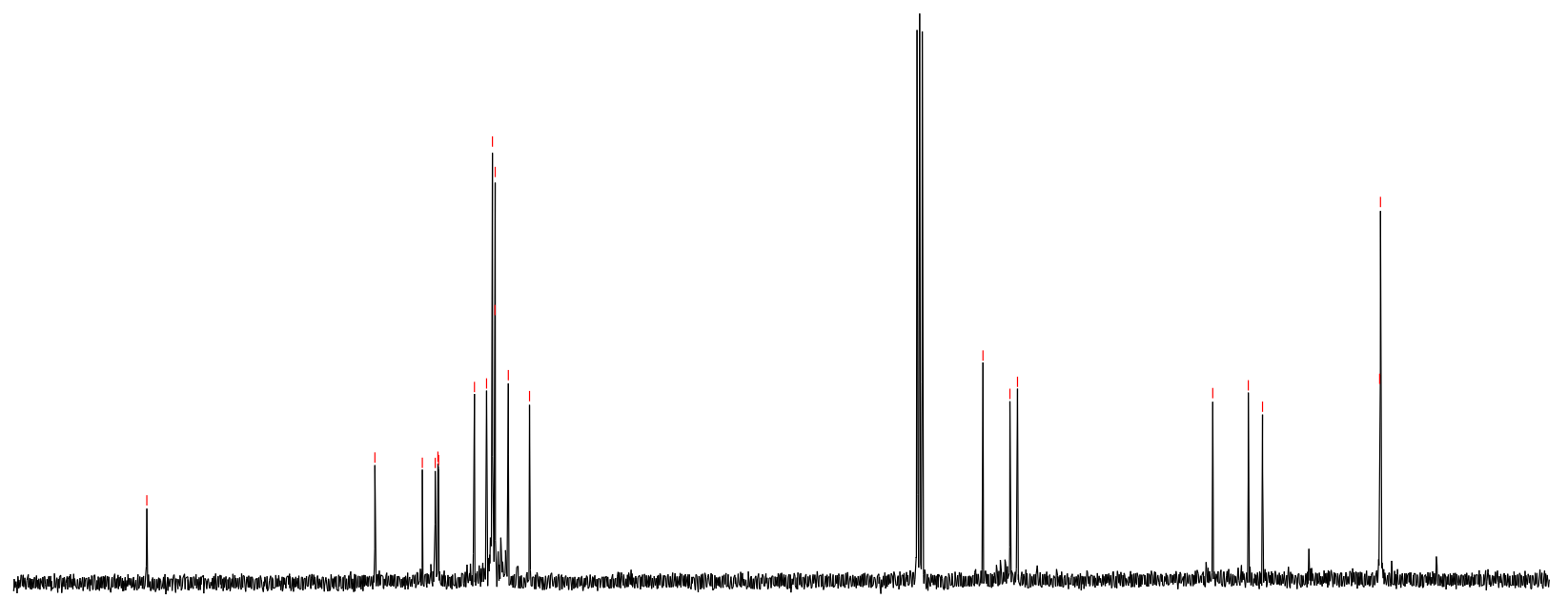

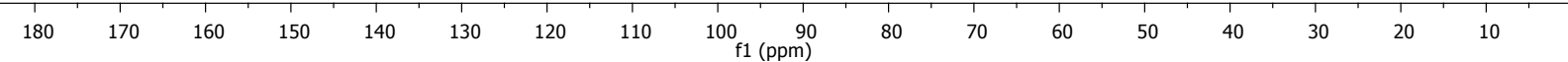



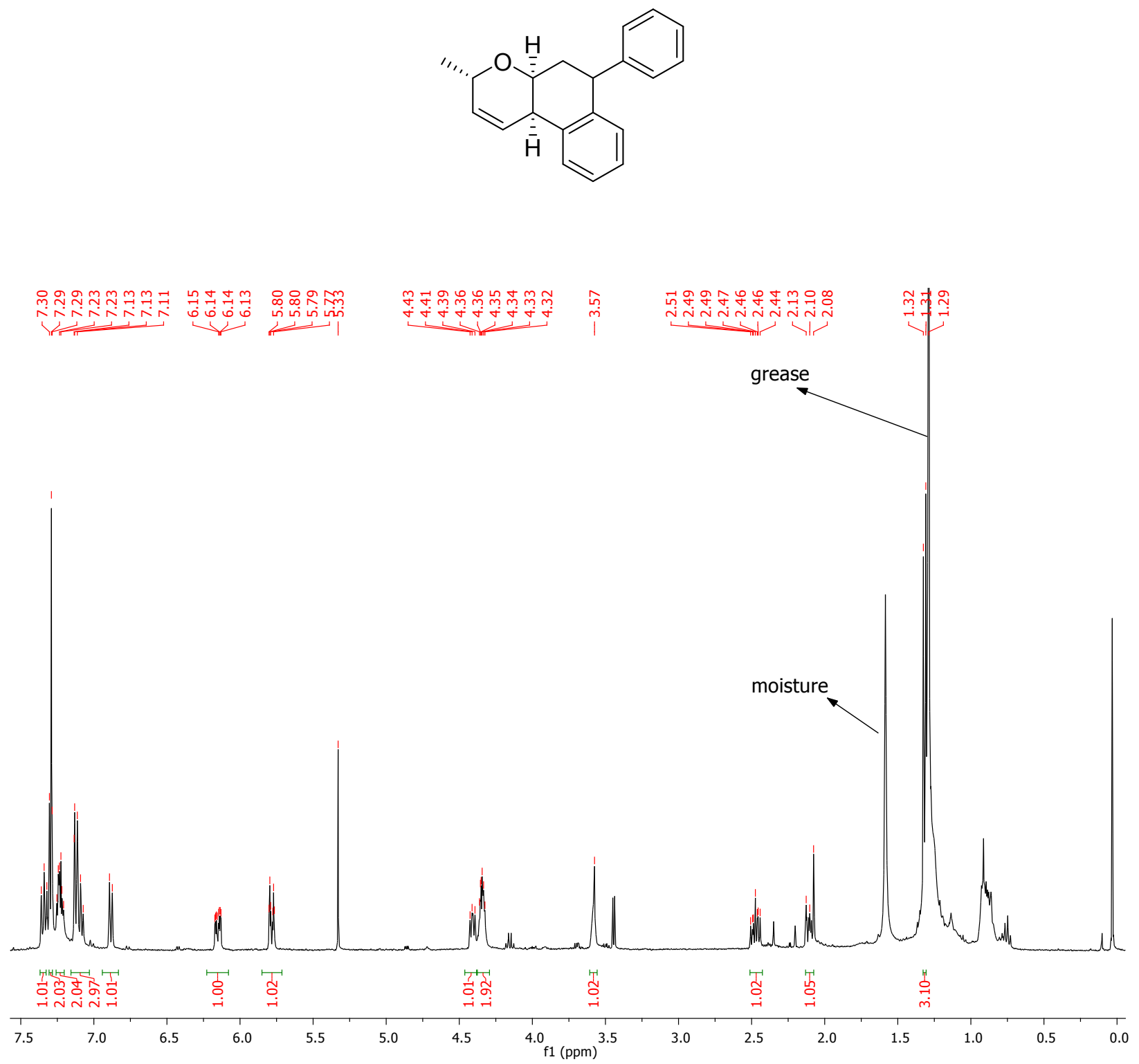
<smiles>C[C@H]1C=C[C@H]2c3ccccc3C(c3ccccc3)C[C@H]2O1</smiles>
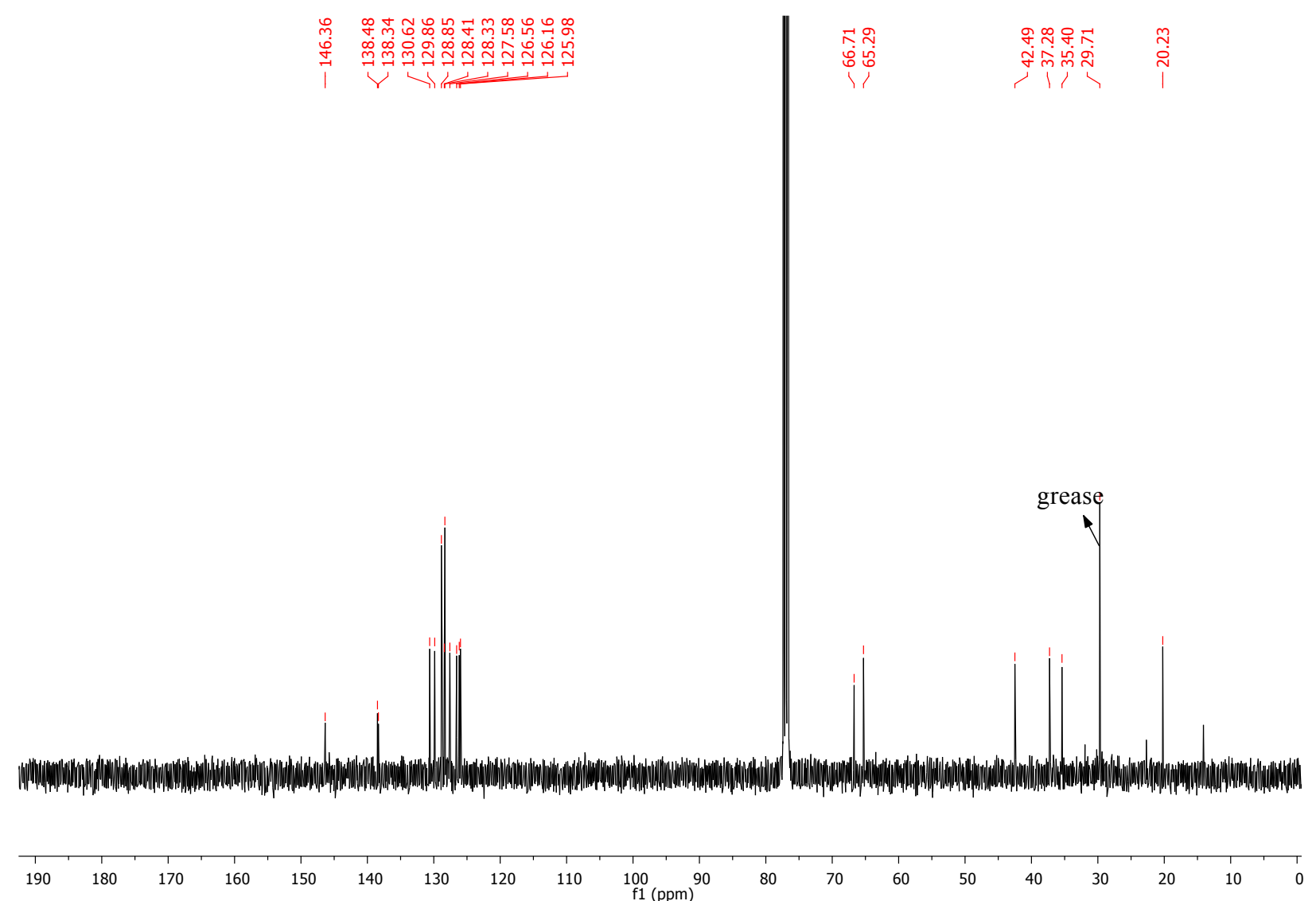
$\underline{{ }^{1} \mathrm{H} \text { NMR (400 MHz, } \mathrm{CDCl}_{3} \text { ) of compound } 7 \mathrm{~g}}$<smiles>CC(=O)CC1C=C[C@H]2c3cccc(Cl)c3C(c3ccc(C)cc3)C[C@H]2O1</smiles>

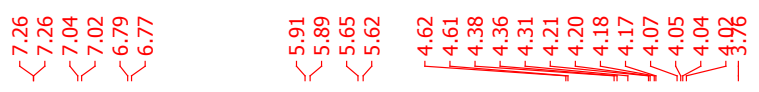

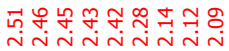

$\underbrace{-14}$

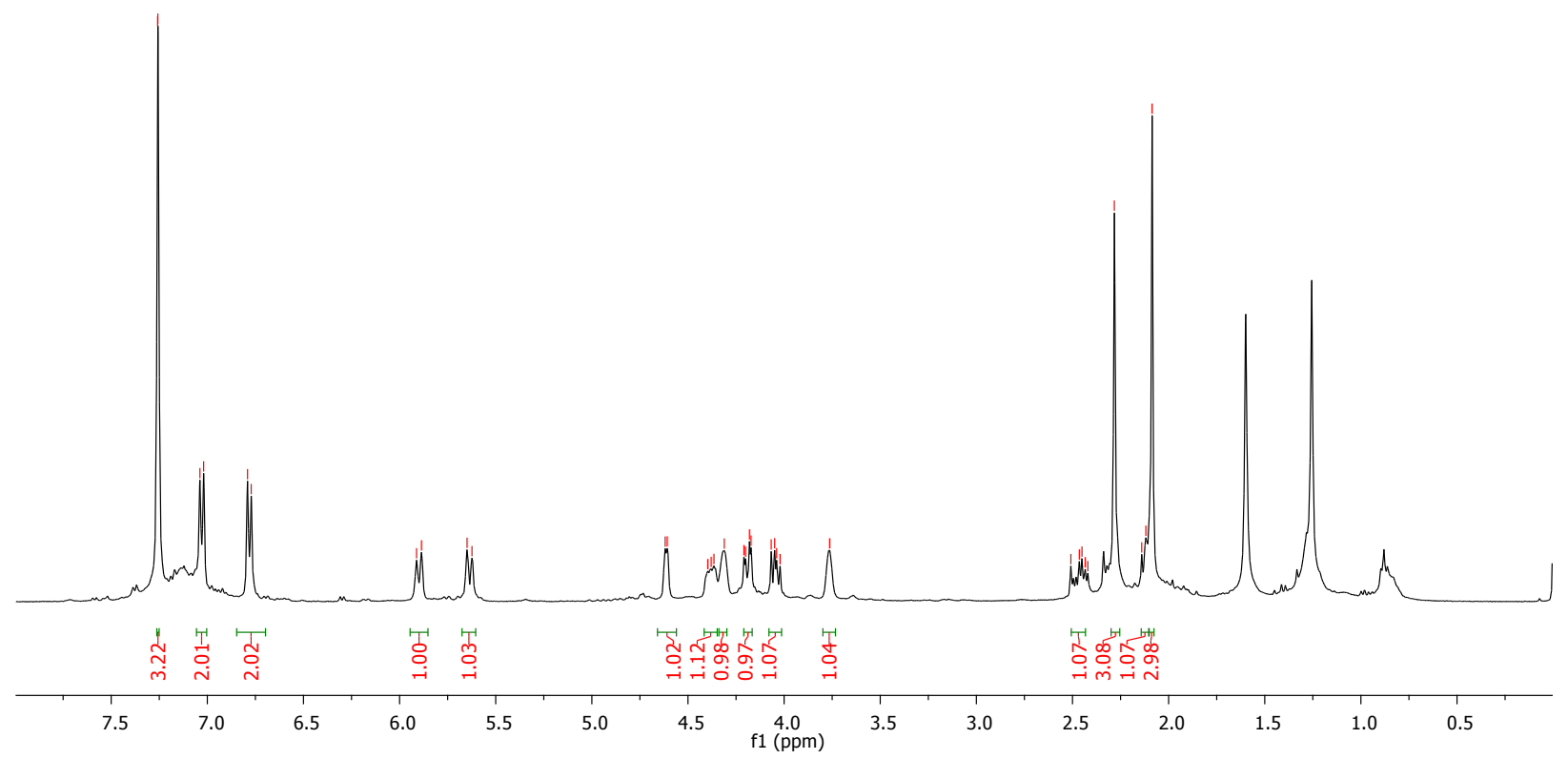


$\underline{{ }^{13} \mathrm{C} \text { NMR (101 MHz, } \mathrm{CDCl}_{3} \text { ) of compound } 7 \mathrm{~g}}$<smiles>CC(=O)OCC1C=C[C@H]2c3cccc(Cl)c3C(c3ccc(C)cc3)C[C@H]2O1</smiles>
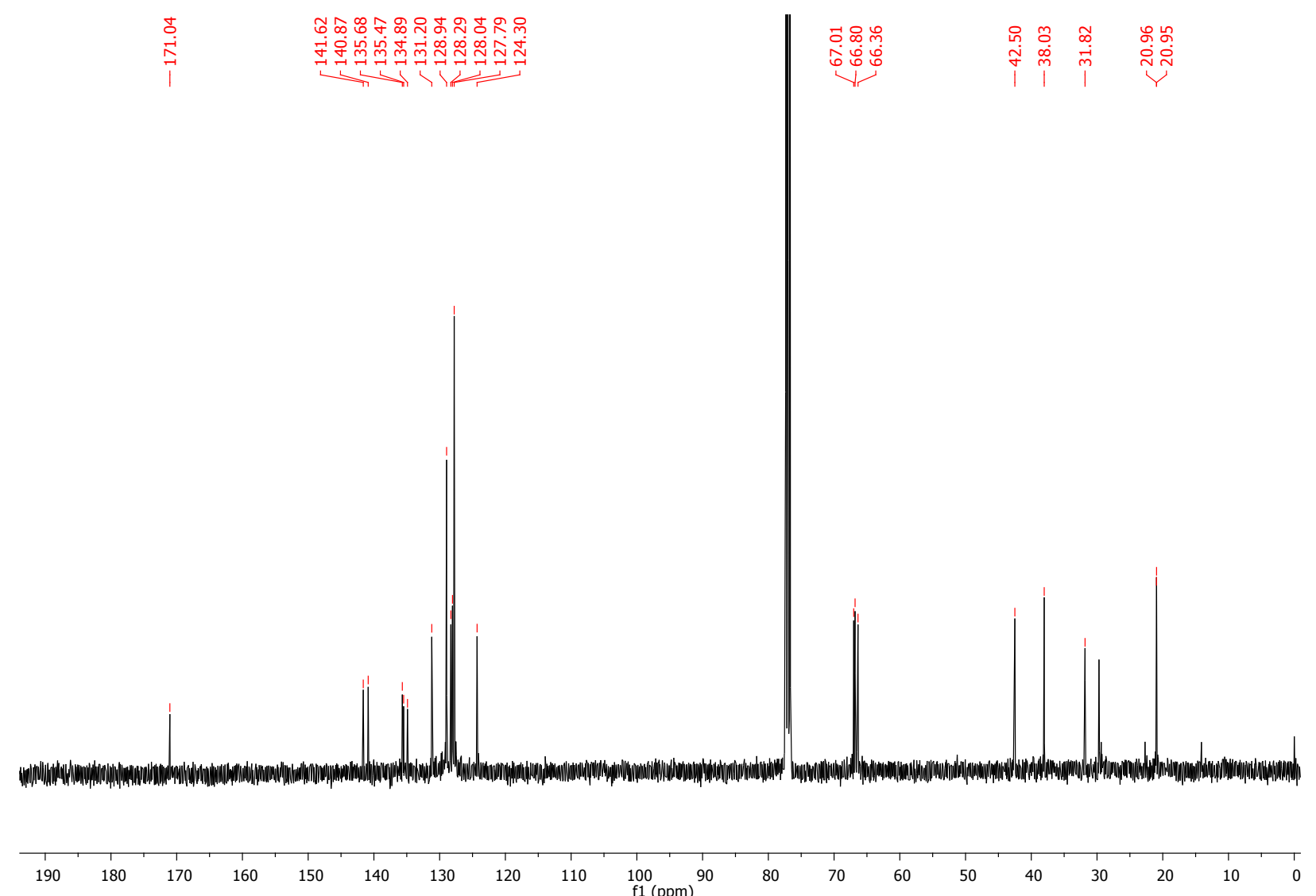


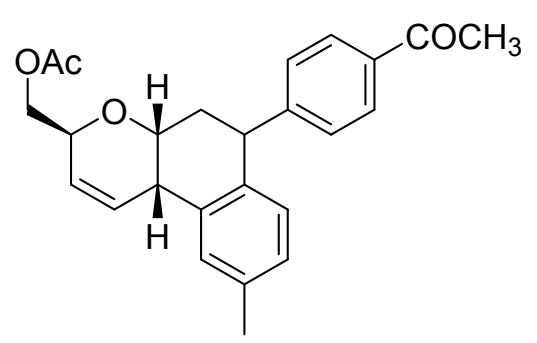

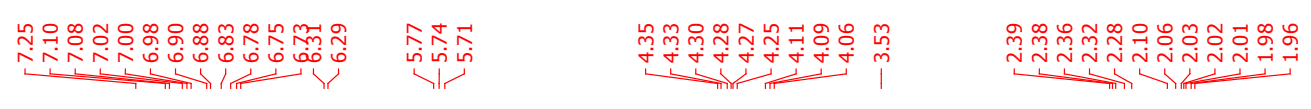

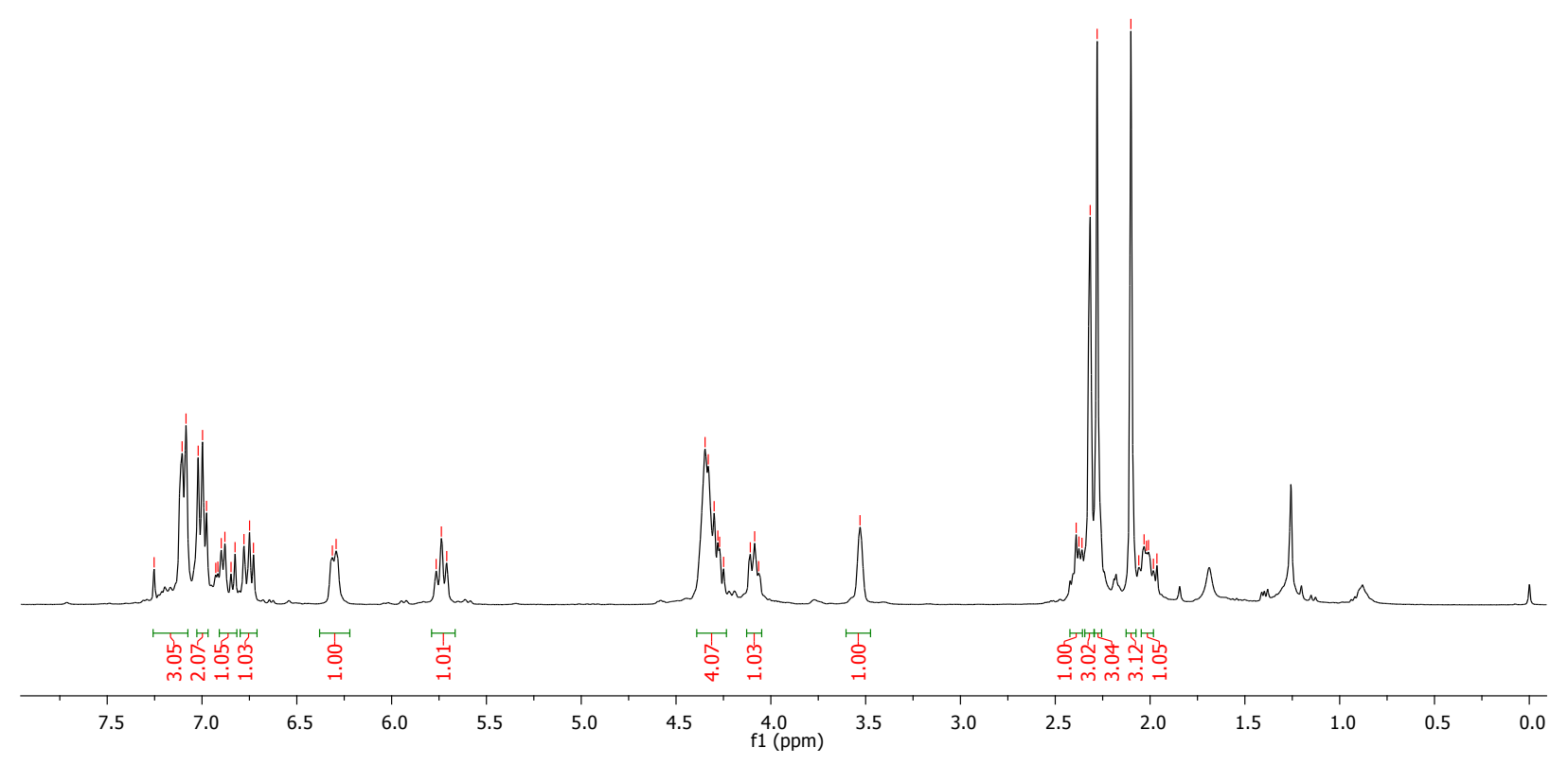



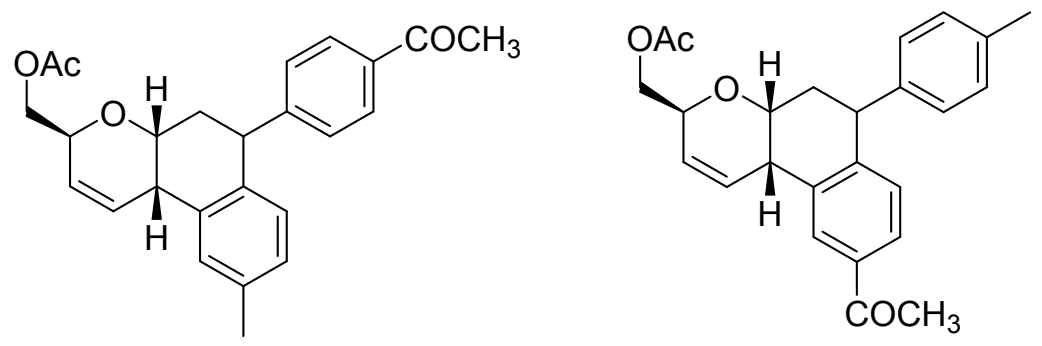

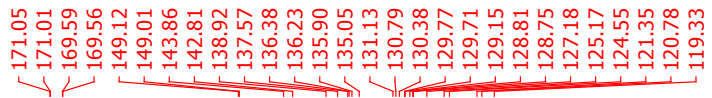

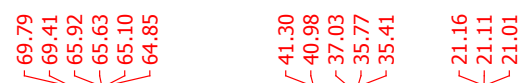

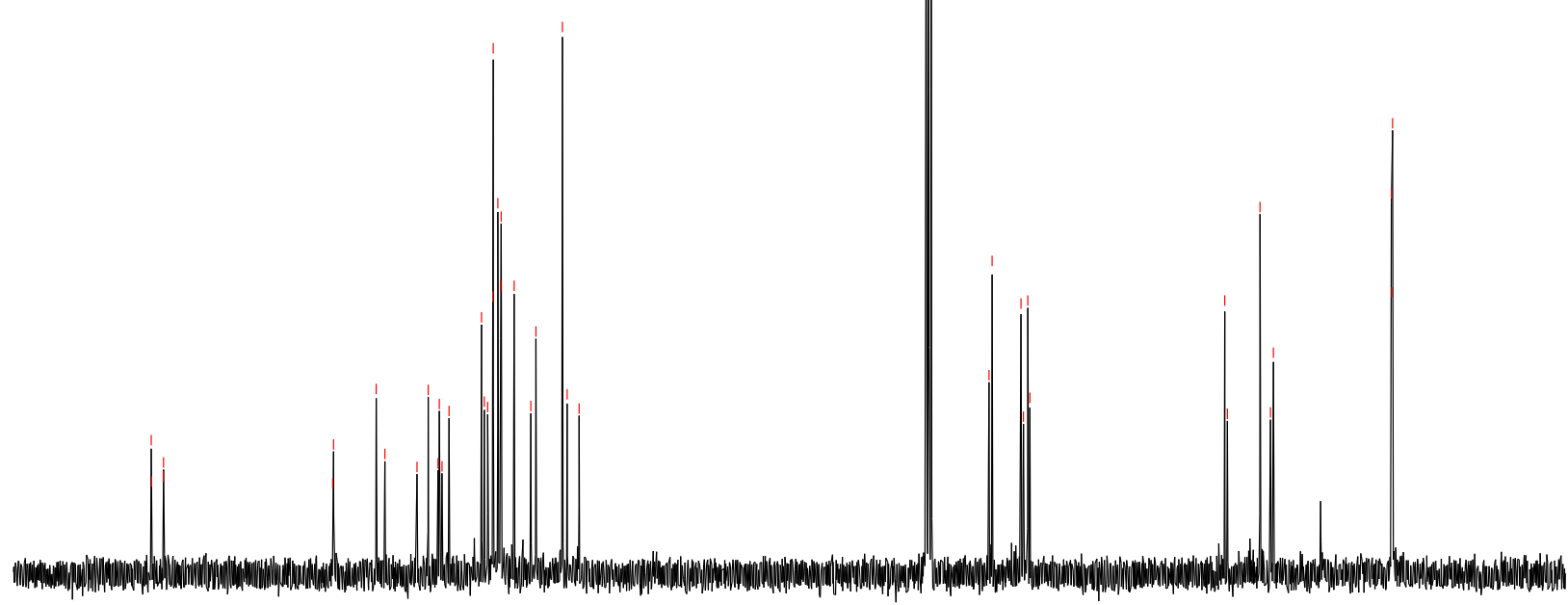

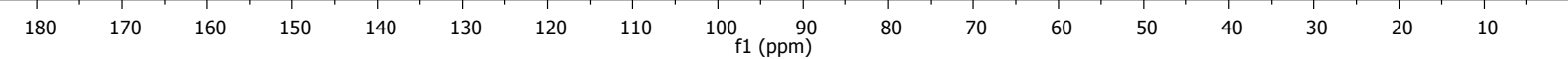


$\underline{{ }^{1} \mathrm{H} N M R\left(400 \mathrm{MHz}, \mathrm{CDCl}_{3}\right) \text { of compound } 7 \mathrm{i}}$
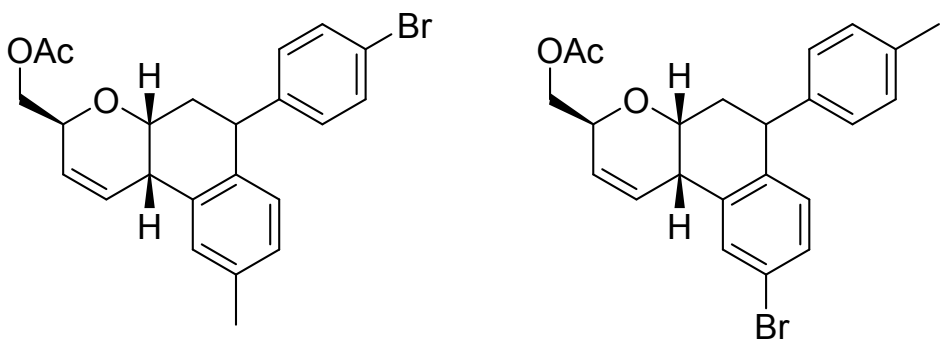

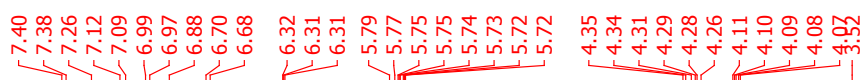

ิㅗㅆ
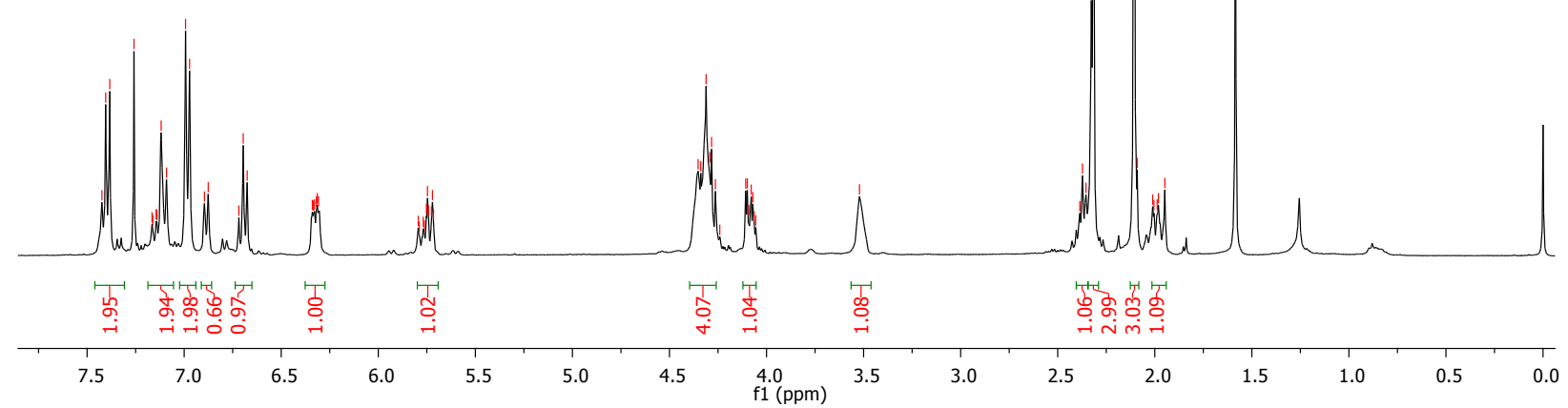
$\underline{{ }^{13} \mathrm{C} \text { NMR (101 MHz, } \mathrm{CDCl}_{3} \text { ) of compound } 7 \mathrm{i}}$
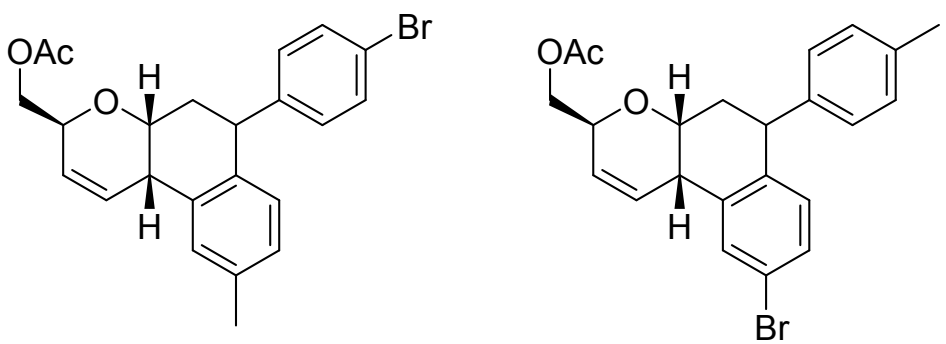

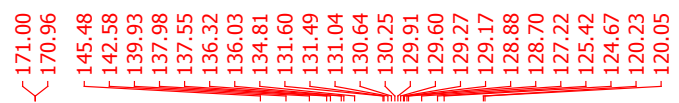
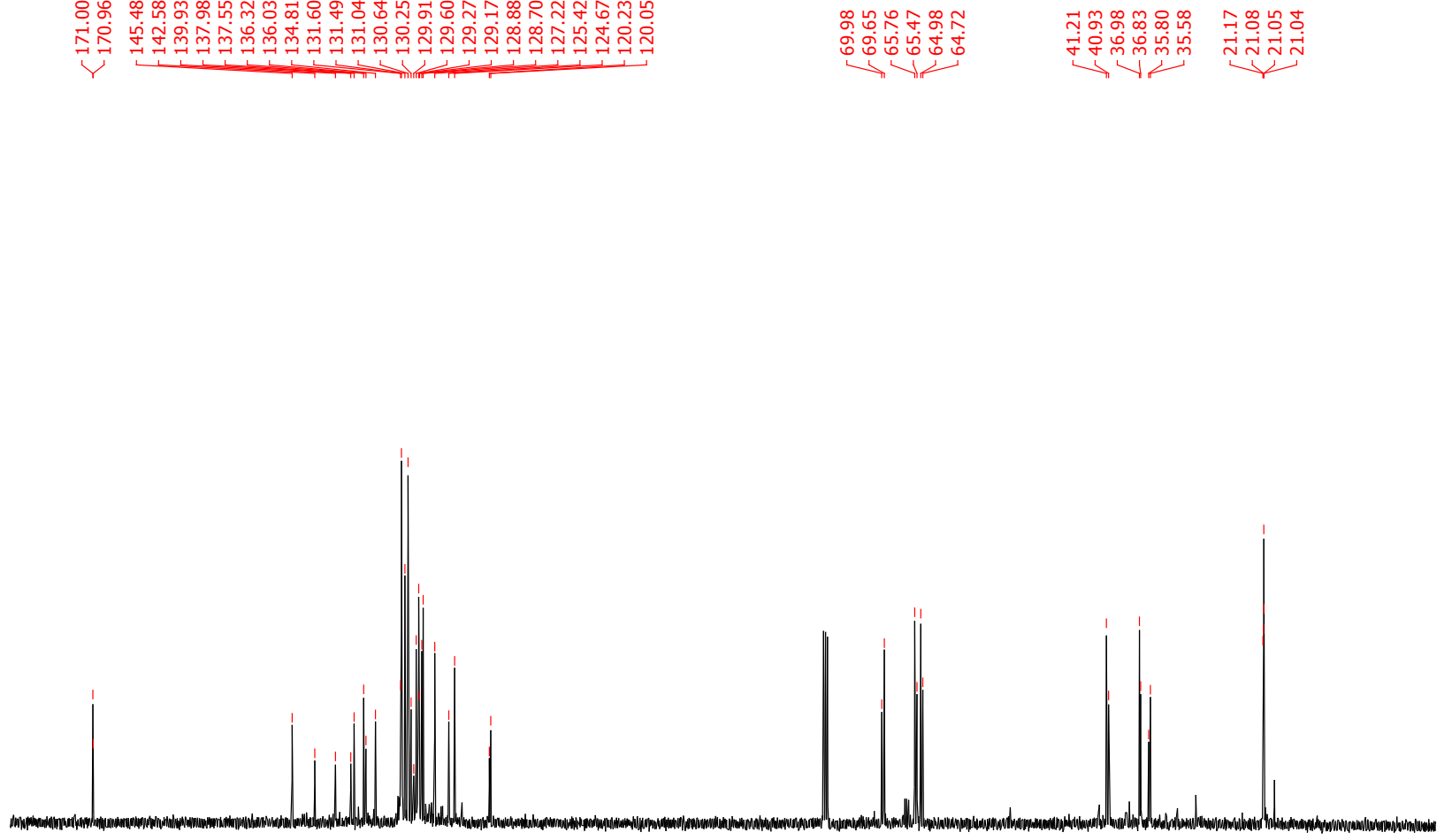

180
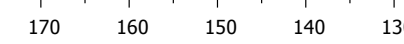

$120 \quad 110 \quad 100 \underset{f 1(\mathrm{ppm})}{90} 80$

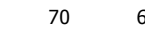



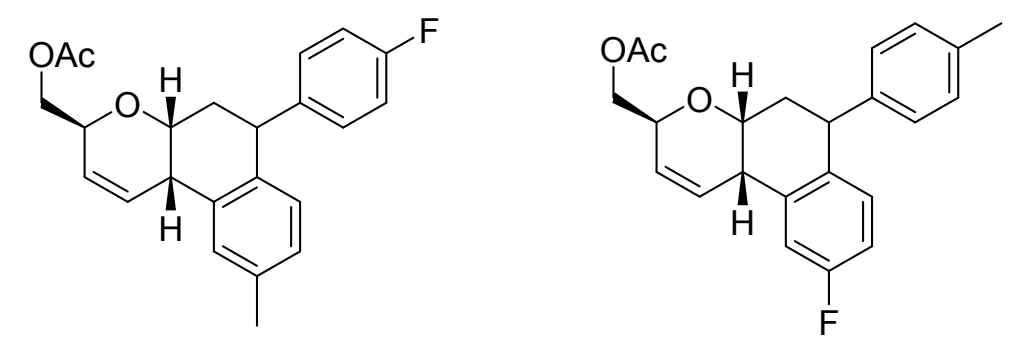

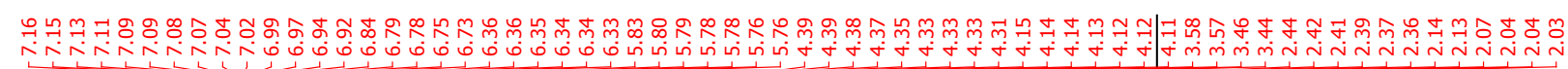

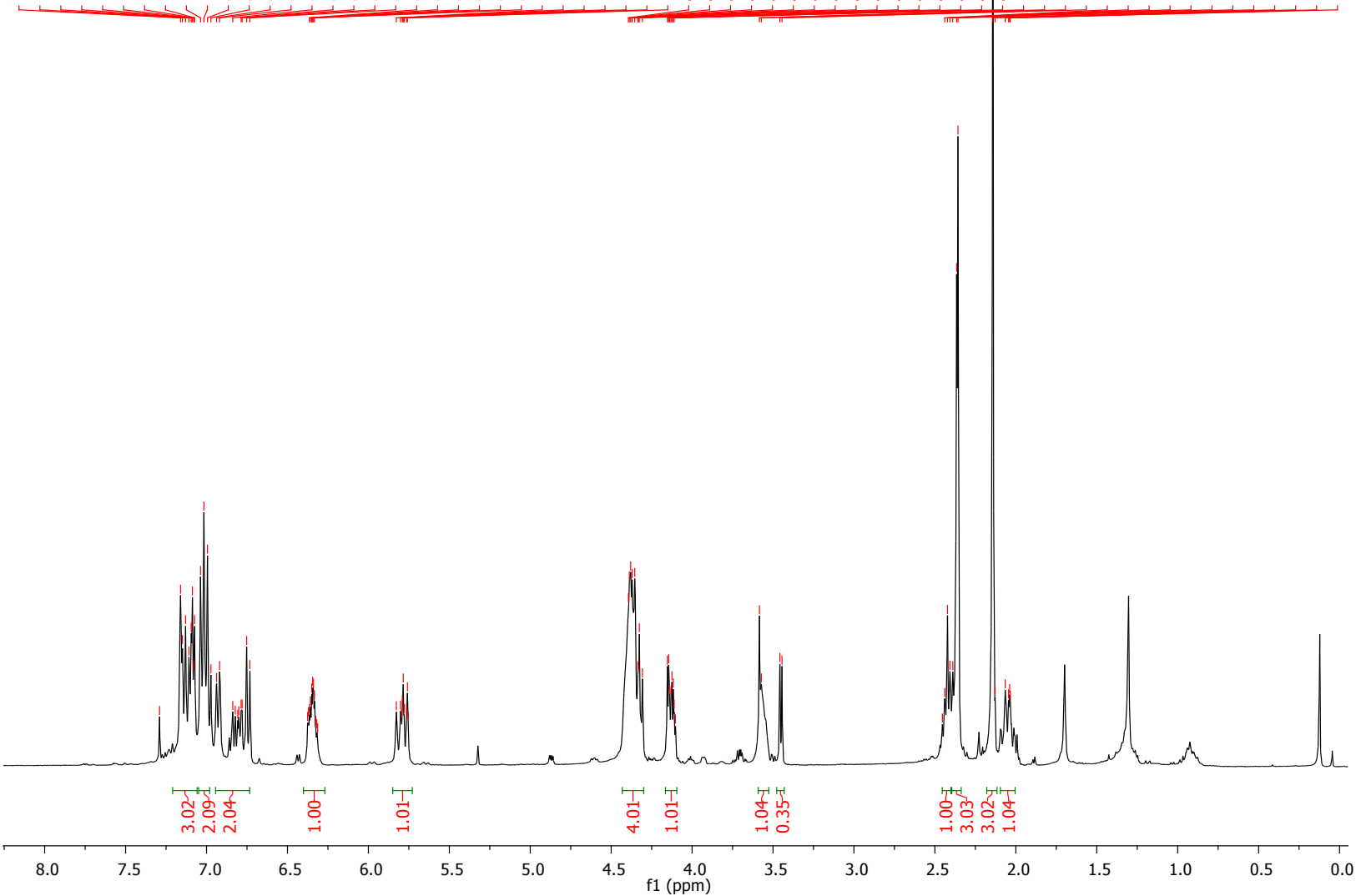



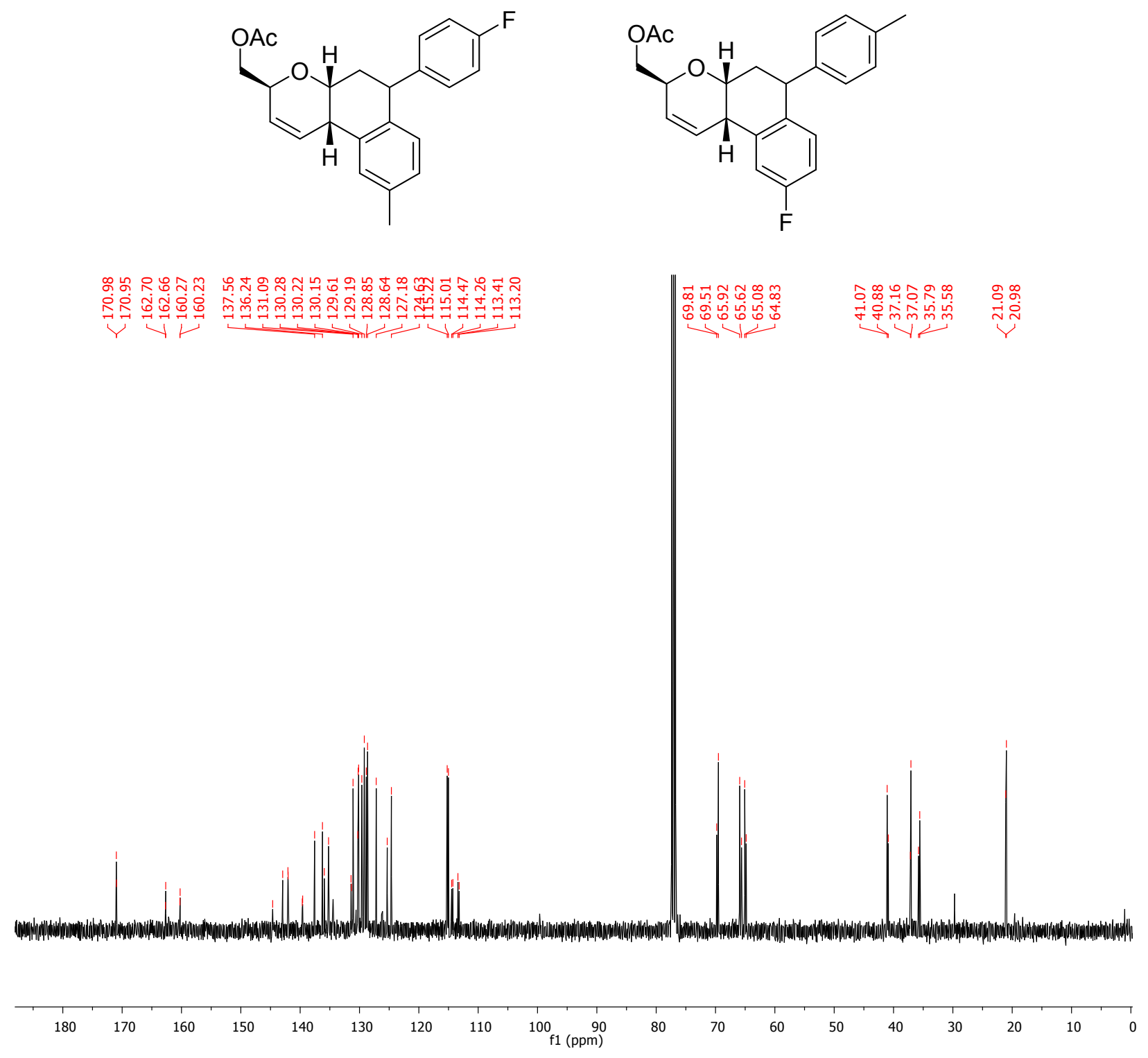

$\underline{1}$ H NMR (400 MHz, $\mathrm{CDCl}_{3}$ ) of compound 7k 

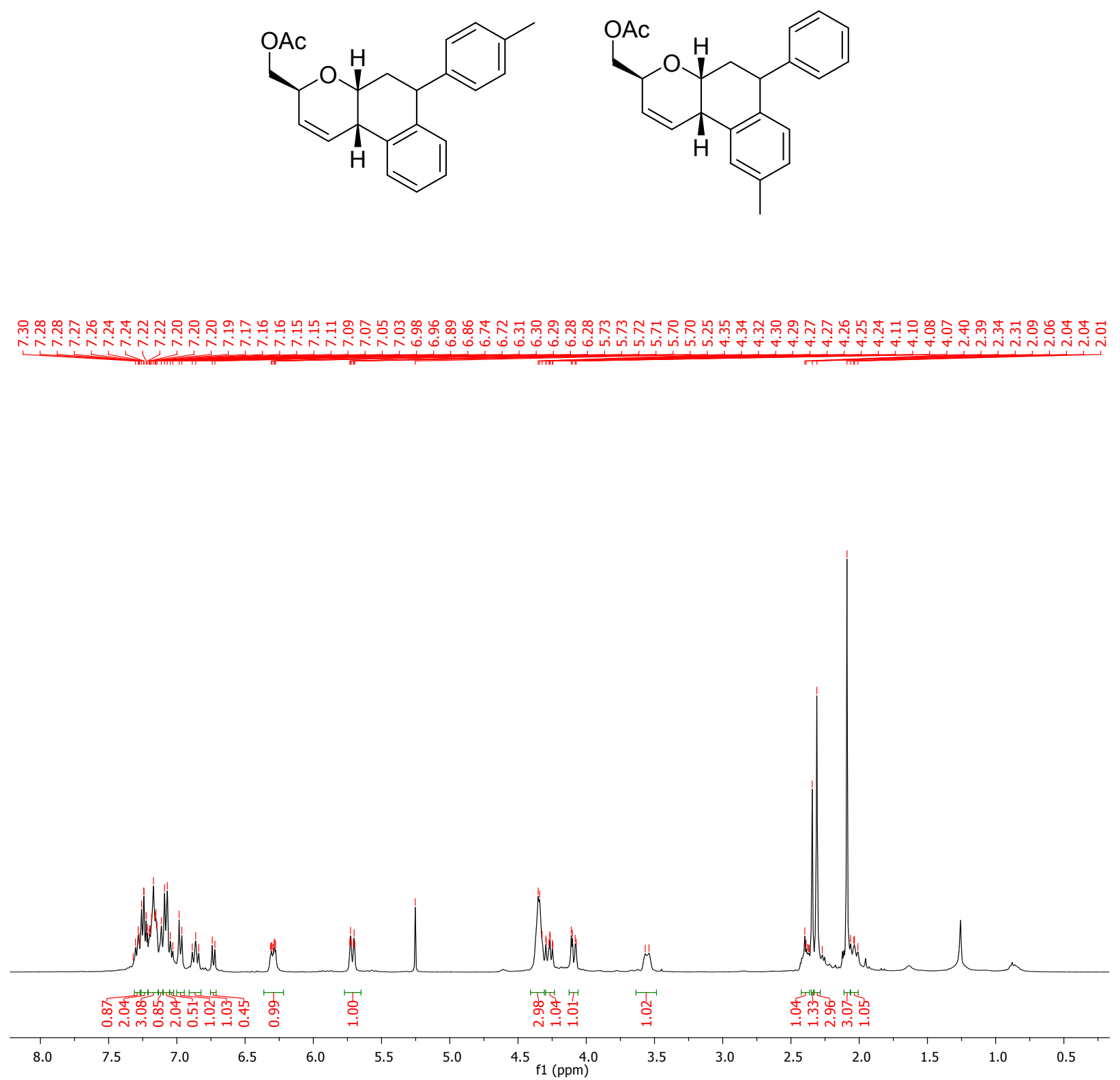


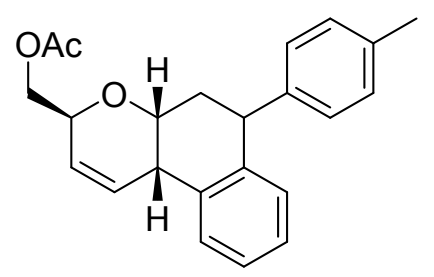

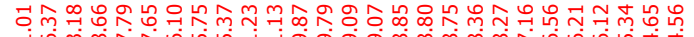

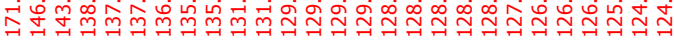

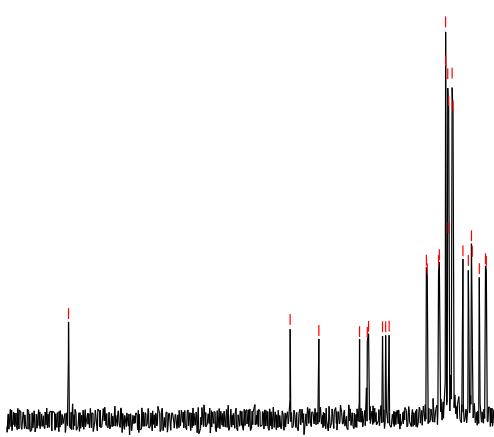

170<smiles>CC(=O)OC[C@H]1C=C[C@H]2c3cc(C)ccc3C(c3ccccc3)C[C@H]2O1</smiles>

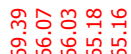

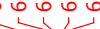

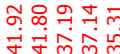

華

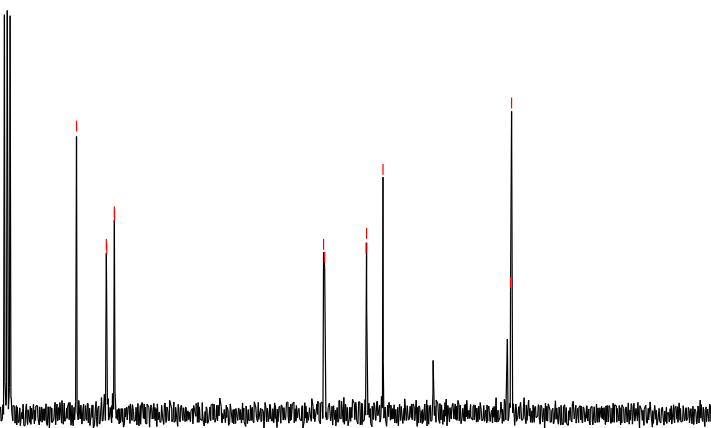

90

$\begin{array}{llll}70 & 60 & 50 & 40\end{array}$


HSQC of compound 7

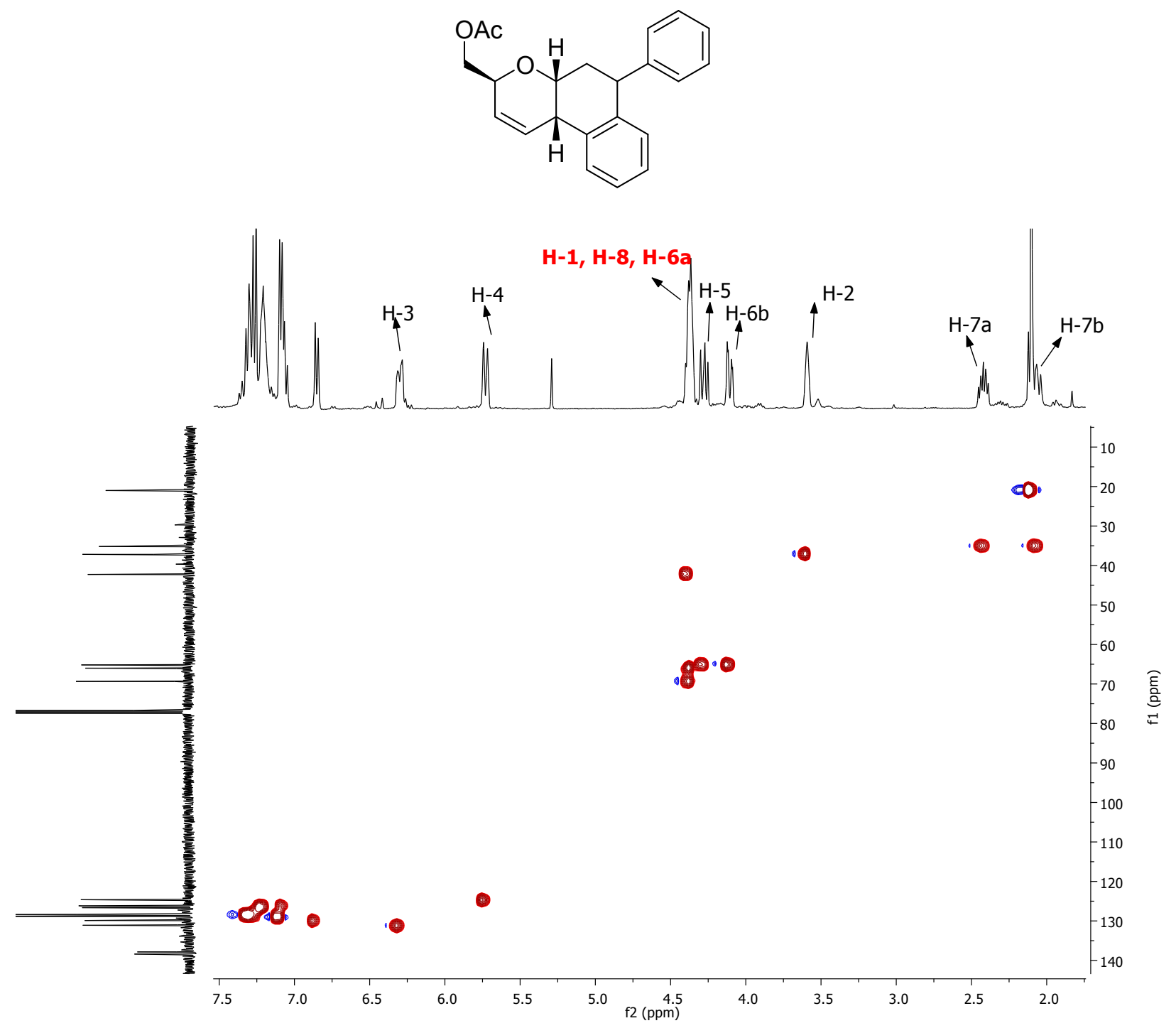


NOESY of compound 7<smiles></smiles>

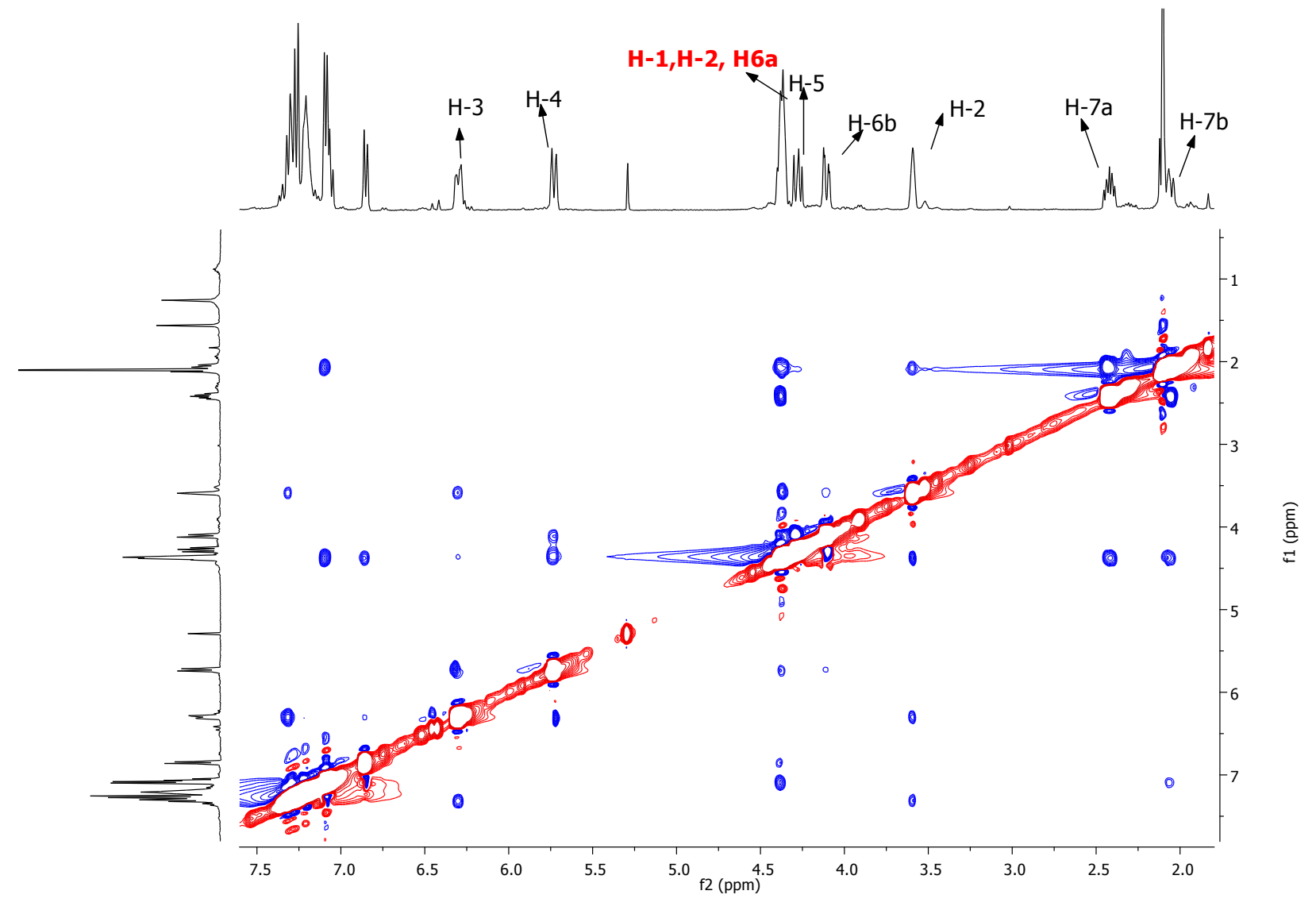

NOESY of deacetylated product of $7 \mathrm{~g}$ 

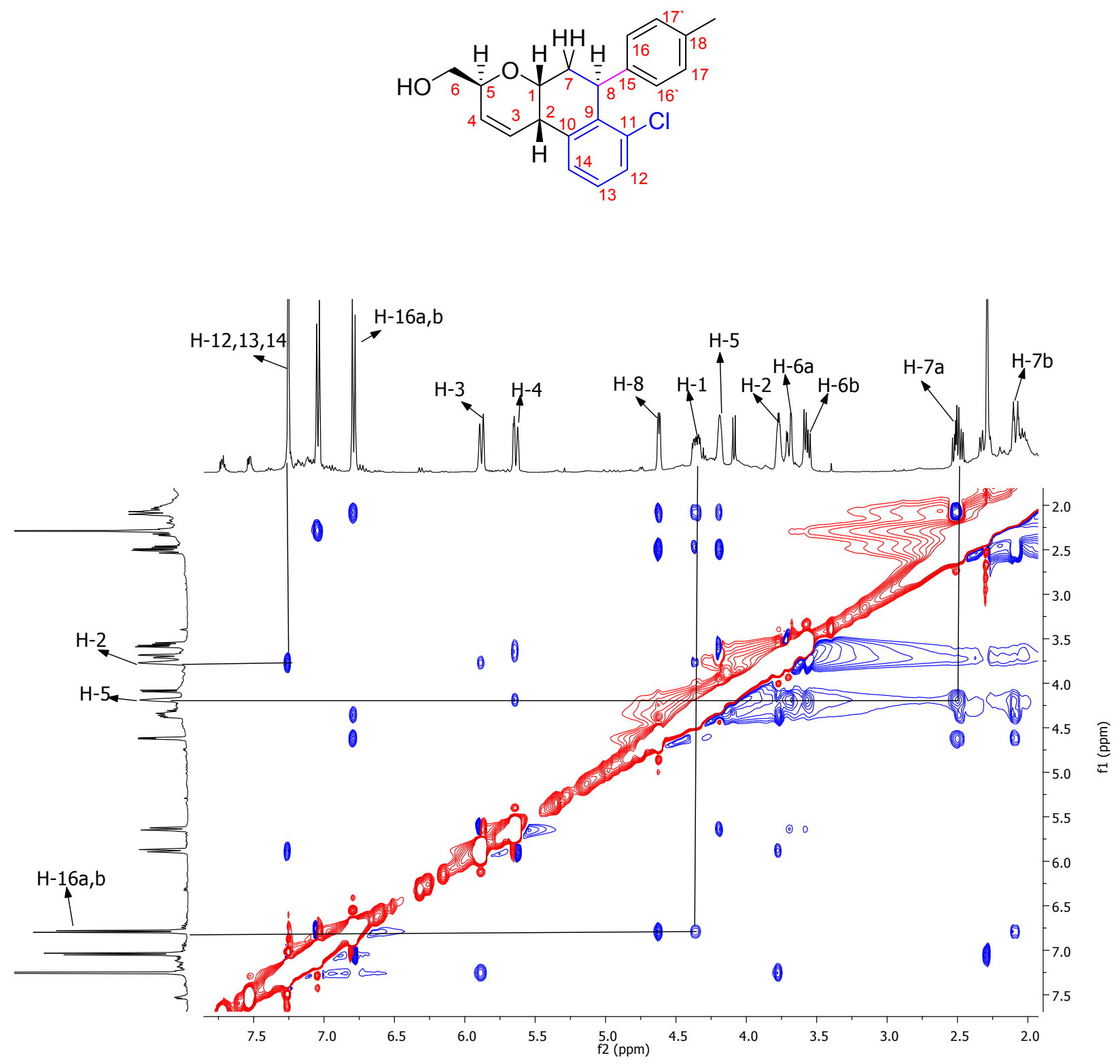

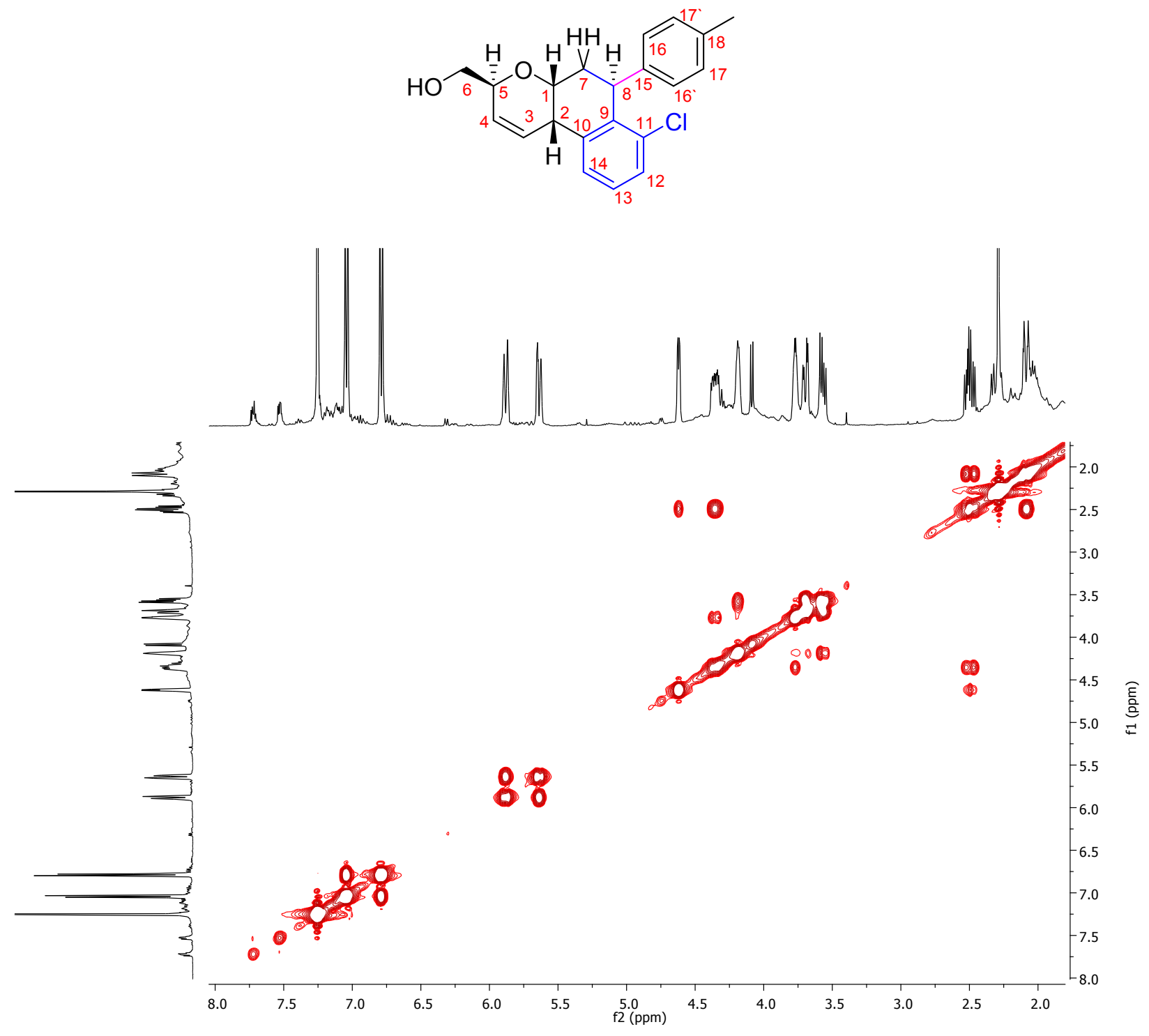

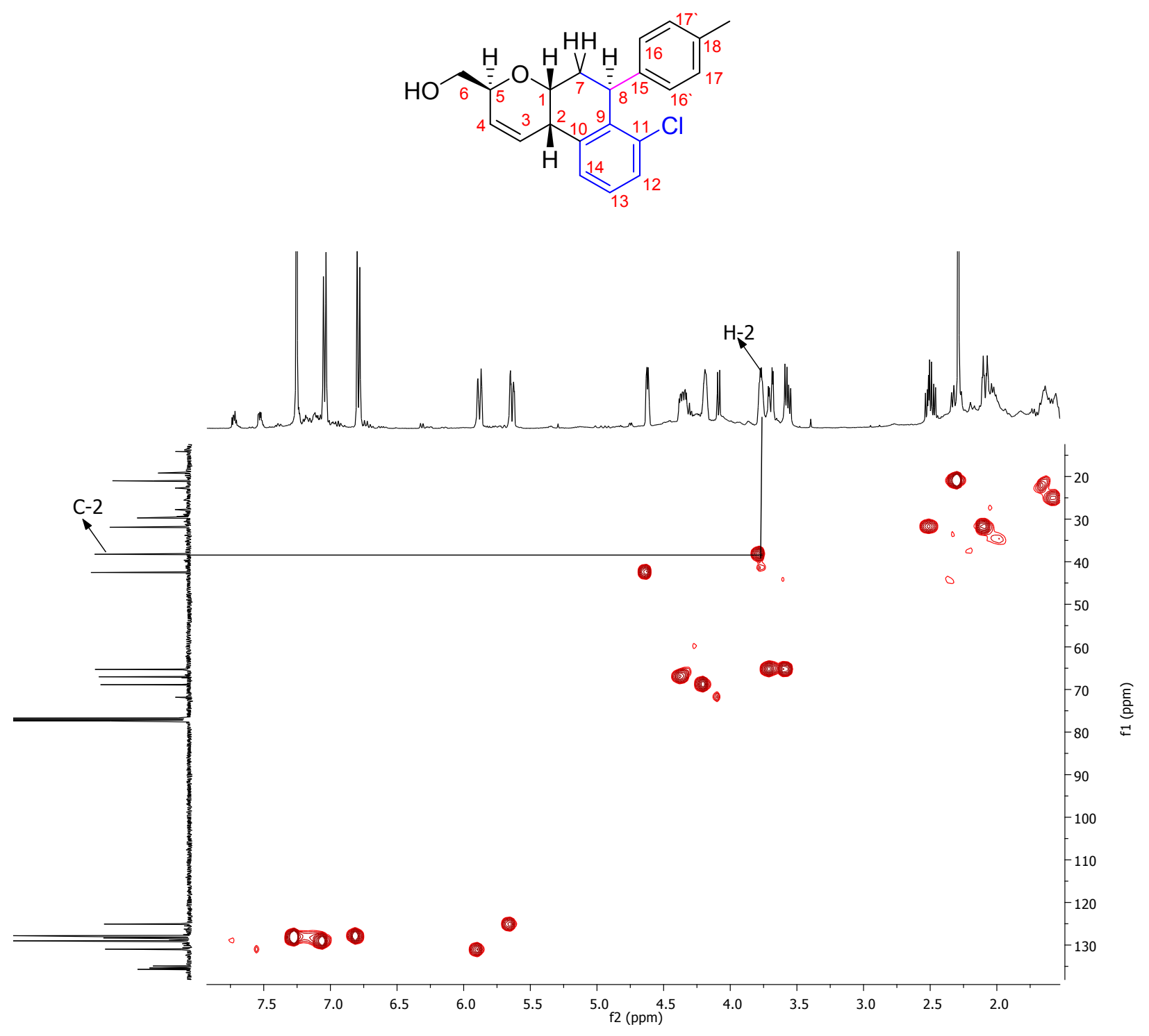
HMBC of deacetylated product of $7 \mathrm{~g}$
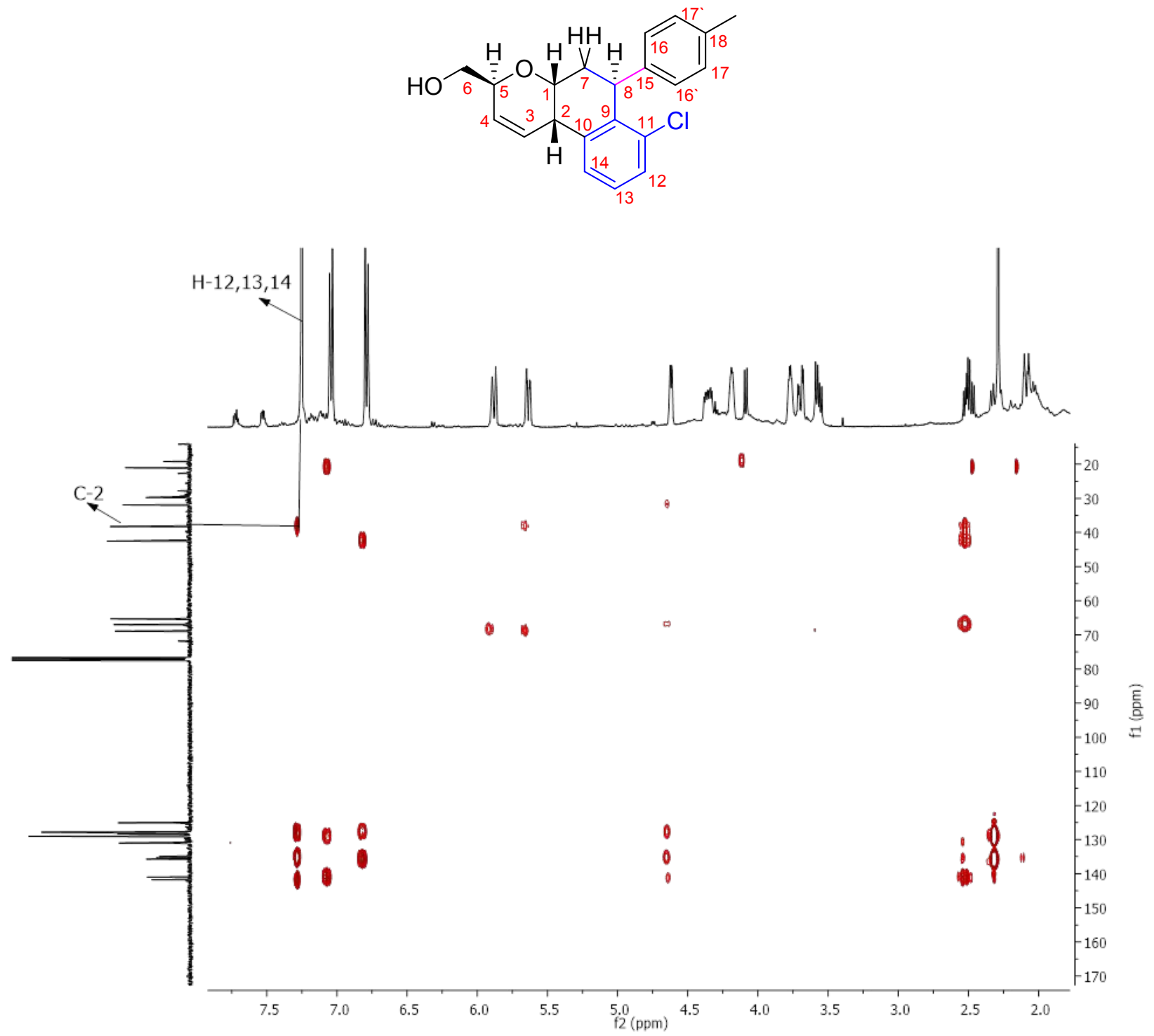